Mathematik

\title{
Obstructions to Stably Fibering Manifolds
}

\author{
Inaugural-Dissertation \\ zur Erlangung des Doktorgrades \\ der Naturwissenschaften im Fachbereich \\ Mathematik und Informatik \\ der Mathematisch-Naturwissenschaftlichen Fakultät \\ der Westfälischen Wilhelms-Universität Münster
}

vorgelegt von

Wolfgang Steimle

aus Mutlangen

$-2010-$ 
Dekan:

Erster Gutacher:

Zweiter Gutacher:
Prof. Dr. Christopher Deninger

Prof. Dr. Wolfgang Lück

apl. Prof. Dr. Michael Joachim

Tag der mündlichen Prüfung: 7. Juli 2010

Tag der Promotion:

7. Juli 2010 


\section{Contents}

$\begin{array}{llr}1 & \text { Introduction } & 5\end{array}$

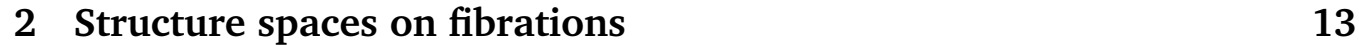

2.1 Definition of the structure space on a fibration . . . . . . . . 14

2.2 Structure spaces as spaces of lifts $\ldots \ldots \ldots \ldots$

2.3 Universal "bundles" . . . . . . . . . . . . . . . . . . . . . 22

3 The parametrized Whitehead torsion 23

3.1 The parametrized $A$-theory characteristic . . . . . . . . . . . . 25

3.2 Definition . . . . . . . . . . . . . . . . . . . . . . 29

3.3 Composition rule and homeomorphism invariance . . . . . . . 34

3.4 The torsion on the stable structure space . . . . . . . . . . . . . 35

3.5 A product formula . . . . . . . . . . . . . . . . 40

3.6 Additivity . . . . . . . . . . . . . . . . . . . 42

3.7 Relation to $h$-cobordisms. . . . . . . . . . . . . . . . . . . 46

3.8 Comparison with the unparametrized case . . . . . . . . . . 50

4 The geometric assembly map $\quad 53$

4.1 Definition of the geometric assembly map . . . . . . . . . . . 53

4.2 Geometric assembly and torsion I . . . . . . . . . . . . . . . . . . 54

4.3 Geometric assembly and torsion II . . . . . . . . . . . . . . 58

$4.4 \quad$ Geometric assembly on bundles of Q-manifolds. . . . . . . . . . 64

4.5 Whitehead torsion on $Q$-manifolds $\ldots \ldots \ldots$. . . . . . 67

\begin{tabular}{|lll}
5 & Applications to fibering questions & 73
\end{tabular}

5.1 Stably fibering manifolds $\ldots \ldots \ldots \ldots$. . . . . . . 73

5.2 Fibering Q-manifolds over compact ANRs . . . . . . . . . . 80

5.3 Change of base and total space . . . . . . . . . . . . . 81

5.4 Examples I: Elementary applications . . . . . . . . . . . . . . . 85

5.5 Examples II: Stable vs. unstable fibering and TOP vs. DIFF . . . . 87

5.6 A spectral sequence $\ldots \ldots \ldots \ldots \ldots$. . . . . . . . . 92

5.7 Examples III: Results of Chapman-Ferry . . . . . . . . . . . . . 96

5.8 Comparison with the obstructions by Farrell-Lück-Steimle . . . . 98 
\begin{tabular}{|lr}
\hline A Some results on fibrations & 101
\end{tabular}

A.1 The fibered homotopy extension property . . . . . . . . . . . . . 101

A.2 Associated fibration and connections . . . . . . . . . . . . 102

A.3 Fiberwise glueing . . . . . . . . . . . . . . . . . . . . 103

A.4 Glueing over different base spaces. . . . . . . . . . . . . . . . . 106

A.5 Tulley's construction . . . . . . . . . . . . . . . . . . . . . 109

A.6 Fill-in for fibrations . . . . . . . . . . . . . . . . . . . . . . . . 111

\begin{tabular}{ll}
\hline Bibliography & 115
\end{tabular} 


\section{Introduction}

Given a map $f: M \rightarrow B$ between closed manifolds, is $f$ homotopic to the projection map of a fiber bundle of closed manifolds? Can the different ways of fibering $f$ be classified? These questions have a long tradition in geometric topology. In the research on high-dimensional manifolds, the investigation of these questions has accompanied the development of the subject since its beginnings: The fibering theorem of Browder-Levine [BL66] was an early application of surgery techniques and the $h$-cobordism theorem. Further results have been obtained by Farrell [Far72] and Siebenmann [Sie70] for $B=S^{1}$, using the $s$-cobordism theorem and computations of the Whitehead group of semi-direct products $G \rtimes_{\alpha} \mathbb{Z}$.

Casson [Cas67] pioneered the study of fibering questions for higher-dimensional base manifolds by considering $B=S^{n}$ by applying techniques of surgery theory. Quinn's thesis [Qui69] was the first to systematically describe block structure spaces using the $L$-theoretic assembly map and to develop a general obstruction theory to "block fibering" a given map.

In the Q-manifold world, Chapman-Ferry [CF78] obtained the most general results available so far. Most recently, in the finite-dimensional case, joint work of the author with Farrell and Lück [FLS09] shows how the obstructions defined by Farrell and Siebenmann over $S^{1}$ can be generalized to arbitrary base spaces (where they stop being a complete set of obstructions).

In the light of the development of parametrized $h$-cobordism theory since the 1970s, this work re-focuses on the role of algebraic $K$-theory in fibering questions. As we will see, higher algebraic $K$-theory of spaces provides obstructions for both questions of existence and uniqueness. Moreover, the vanishing of these obstructions has a concrete geometric meaning: The obstructions constructed in this work form a complete set of obstructions to stably fibering manifolds. Here stabilization refers to crossing the total space with disks of sufficiently high dimension, thus leaving the category of closed manifolds. In fact, the theory of stably fibering manifolds is best formulated and proved entirely in the world of compact manifolds with boundary (which we call compact manifolds for short). 
More concretely, let $f: M \rightarrow B$ be a map between compact topological manifolds. Then, by definition, $f$ stably fibers if, for some $n \in \mathbb{N}$, the composite

$$
f \circ \text { Proj: } M \times D^{n} \rightarrow M \rightarrow B
$$

is homotopic to the projection map of a fiber bundle whose fibers are compact topological manifolds. The following questions will be dealt with:

- When does $f$ stably fiber?

- How many different ways are there for $f$ to stably fiber? Denote by $C$ the set of all bundle maps $g: M \times D^{n} \rightarrow B$ for some $n$ which are homotopic to $f \circ$ Proj. We define two elements to be equivalent, and write $g \sim g^{\prime}$, if after further stabilizing the two bundle maps $g$ and $g^{\prime}$ are isomorphic through a homeomorphism $i: M \times D^{N} \rightarrow M \times D^{N}$ (i.e. $i \circ g=g^{\prime}$ ), such that $i$ is homotopic to the identity map. The precise question is then: How can $C / \sim$ be described?

Factor $f$ into a homotopy equivalence $\lambda$ followed by a fibration $p$. Under a finiteness assumption on the fibers $F_{b}$ of $p$, two obstructions will be defined:

(i) $\operatorname{Wall}(p) \in H^{0}\left(B ; \mathrm{Wh}\left(F_{b}\right)\right)$, which is an obstruction to reducing $p$ to a fiber bundle of compact manifolds. Here the term Wh is used to denote the (connective topological) Whitehead spectrum as defined by Waldhausen. It is defined in terms of higher algebraic $K$-theory of spaces and is closely connected to the classification of parametrized $h$-cobordisms. The term $H^{0}\left(B ; \mathrm{Wh}\left(F_{b}\right)\right)$ denotes a specific generalized cohomology group of $B$ with respect to the Whitehead spectrum of the fibers, where the coefficients are twisted according to the data of the fibration $p$.

(ii) If Wall $(p)$ vanishes, then there is a second obstruction $o(f)$ lying in a quotient of the Whitehead group Wh $\left(\pi_{1} M\right)$.

See section 5.1 for the precise explanation of terms.

Theorem 1.1 (Existence). The map $f$ stably fibers if and only if the fibers of $p$ are homotopy finitely dominated, and Wall $(p)$ and $o(f)$ both vanish.

Theorem 1.2 (Classification). If $f$ stably fibers, then the set $C / \sim$ is in bijection with a specific subgroup of $H^{0}\left(B ; \Omega \mathrm{Wh}\left(F_{b}\right)\right)$.

Again, see section 5.1 for formal statements and proofs. All the results are proved in the category of topological manifolds, while fibering of compact $Q$-manifolds is also treated. See Remark 5.5 for some comments on the differentiable case. 


\section{Outline of the proof}

The above results on the stable fibering problem are formal consequences of corresponding results on certain structure spaces, together with a theorem by Dwyer-Weiss-Williams [DWW03]. We will be particularly interested in the structure space $\mathscr{S}_{n}(p)$ of $n$-dimensional compact manifold structures on a fibration $p: E \rightarrow B$. A point $x \in \mathscr{S}_{n}(p)$ corresponds to a fiber bundle $q: M \rightarrow B$ whose fibers are compact topological manifolds, and that comes equipped with a fiber homotopy equivalence $p \rightarrow q$. If $B$ is a point, this is just the usual definition of the ("honest") structure space on a space $E$. However, notice that there are no extra conditions on the boundary, in contrast to surgery theory.

Structure spaces on fibrations are more interesting than usual structure spaces in that they allow pairings: For fibrations $p: E \rightarrow B$ and $q: B \rightarrow B^{\prime}$, there is an obvious map

$$
\mathscr{S}_{n}(p) \times \mathscr{S}_{k}(q) \rightarrow \mathscr{S}_{n+k}(q \circ p)
$$

For example, if we take $B^{\prime}$ to be a point, then the map relates the structure spaces of $B$ and $p$ with the structure space of $E$. In this situation, whenever $p$ is provided with a canonical structure we obtain a transfer map from the structure space of $B$ to the one of $E$.

More interesting for us however will be the case where the space $B$ is equipped with a canonical structure. In this case we get a map

$$
\alpha: \mathscr{S}_{n}(p) \rightarrow \mathscr{S}_{n+k}(E)
$$

which geometrically assembles the structures of all the fibers to get one big structure on the total space. Therefore we are going to call $\alpha$ the geometric assembly map. (This notion should not be confused with the homotopytheoretically defined assembly maps in $K$ - and $L$-theory.)

The connection of this notion to the fibering problem is given by the following observation: Given $f: M^{n+k} \rightarrow B^{k}$, again factor $f$ into a homotopy equivalence $\lambda: M \rightarrow E$ followed by a fibration $p$. Then $f$ fibers if and only if the element in $\pi_{0} \mathscr{S}_{n+k}(E)$ given by the class of $\lambda$ "disassembles", i.e. is in the image of $\pi_{0}(\alpha)$.

Thus it is desirable to find a more accessible description of the map $\alpha$ from a computational point of view. One of the main results of this work is to give such a description after stabilization. To achieve this, we will make use of two invariants of the structure space of a compact manifold $M$. On the one hand, higher algebraic $K$-theory can be used to define a "parametrized Whitehead 
torsion" map

$$
\tau: \mathscr{S}_{n}(M) \rightarrow \Omega \mathrm{Wh}(M),
$$

which factors through the stabilization process given by crossing with unit intervals. On the other hand the tangent bundle defines a map

$$
T: \mathscr{S}_{n}(M) \rightarrow \operatorname{map}(M, B \mathrm{TOP}(n))
$$

which factors through stabilization if we allow ourselves to stabilize the righthand side as well.

Combining these two invariants produces a map

$$
\tau \times T: \mathscr{S}(p):=\operatorname{colim}_{n} \mathscr{S}_{n}(p) \rightarrow \Omega \mathrm{Wh}(M) \times \operatorname{map}(M, B \mathrm{TOP}) .
$$

It follows from a result of Hoehn [Hoe09] (which in turn builds on the parametrized $h$-cobordism theorem of Waldhausen-Jahren-Rognes [WJR08]) that this map is a weak homotopy equivalence.

Now if $p: E \rightarrow B$ is a bundle of compact topological manifolds, then parametrized versions of the torsion and the tangent bundle maps can be defined. Similarly to above, there results a weak homotopy equivalence

$$
\tau \times T_{\text {fib }}: \mathscr{S}(p) \stackrel{\simeq}{\rightarrow} \Gamma\left(\begin{array}{c}
\Omega \mathrm{Wh}_{B}(E) \\
\downarrow \\
B
\end{array}\right) \times \operatorname{map}(E, B \mathrm{TOP}) .
$$

(See section 3 for notation. The range of the map $\tau$ is, roughly, the space of sections of the fibration obtained from $p$ by applying the functor $\Omega$ Wh fiberwise. The map $T_{\text {fib }}$ is given by the fiberwise tangent bundle.)

Now that we have an algebraic (or better homotopy-theoretic) description of the geometrically defined stable structure spaces, we would like to algebraically describe the geometric assembly map. Suppose for simplicity that $B$ is connected. Choose $b \in B$, and consider the composite

$$
\alpha: \Gamma\left(\begin{array}{c}
\Omega \mathrm{Wh}_{B}(E) \\
\downarrow \\
E
\end{array}\right) \stackrel{\text { Restr. }}{\longrightarrow} \Omega \mathrm{Wh}\left(F_{b}\right) \stackrel{\chi_{e}(B) \cdot i_{*}}{\longrightarrow} \Omega \mathrm{Wh}(E)
$$

where $\chi_{e}(B)$ is the Euler characteristic of $B$ and $i_{*}$ the map induced by the inclusion from the fiber into the total space. Furthermore, from the $H$-space structure on BTOP we get a map

$$
+p^{*} \text { TB }: \operatorname{map}(E, B \text { TOP }) \rightarrow \operatorname{map}(E, B T O P) .
$$


Theorem 1.3. The following diagram commutes up to homotopy:

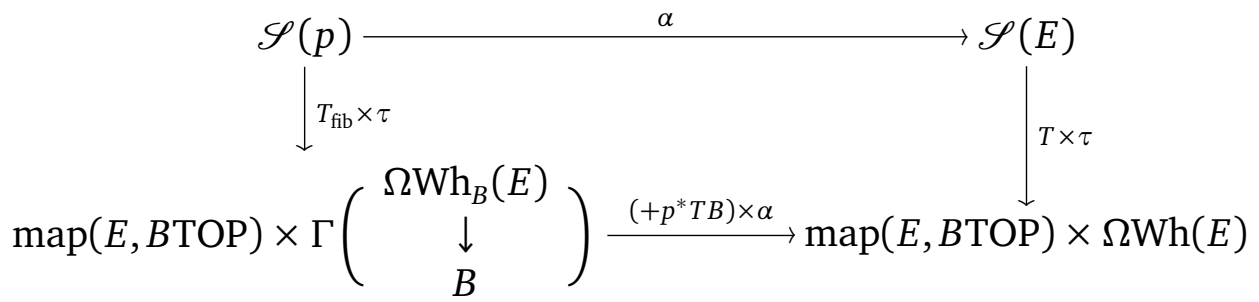

In summary, the lower horizontal map in the diagram can be thought of as an algebraic description of the geometric assembly map appearing in the upper line. The proof of Theorem 1.3 uses properties of the parametrized Whitehead torsion with respect to composition, glueing, and taking cartesian product with contractible manifolds. These properties are proved in section 3 . Building on these results, one can verify the commutativity of the diagram inductively on a handlebody decomposition of $B$. This is done in section 4 .

Returning to the fibering questions, recall the factorization $f=p \circ \lambda$ of our map into a homotopy equivalence and a fibration. If the fibers of $p$ are homotopy finitely dominated, the work of Dwyer-Weiss-Williams gives a criterion in terms of the algebraic $K$-theory of spaces whether $p$ admits a reduction to a fiber bundle of compact manifolds. This leads to the definition of the first obstruction in a straightforward way.

Hence if this obstruction is zero, then we may assume that $p$ is actually a fiber bundle of compact manifolds. In this case, the total space of $p$ is a compact manifold, so the Whitehead torsion $\tau(\lambda)$ is defined. Its class in the cokernel of $\pi_{0}(\alpha)$ is independent of the choice of the bundle $p$ and defines the second obstruction $o(f)$. Now it follows from Theorem 1.3 that $f$ stably fibers if and only if $o(f)=0$. The proof of Theorem 1.2 runs along the same lines, showing that the different possible ways of stably fibering $f$ is in bijection with the kernel of $\pi_{0}(\alpha)$.

It should be mentioned that there is a slightly different, more geometric way to prove Theorem 1.1 that does not make use of Hoehn's result. Instead one can directly conclude from the parametrized $h$-cobordism theorem that $H^{0}\left(B ; \Omega \mathrm{Wh}\left(F_{b}\right)\right)$ may be identified with the isomorphism classes of "stable fiberwise $h$-cobordisms on $p$ ". Then the idea of proof is the following: Supposing $\operatorname{Wall}(p)=0$, we may factor $f=p \circ \lambda$ where $p$ is a fiber bundle and $\lambda$ is a stably tangential homotopy equivalence. The fact that $o(f)$ is zero means that, by definition, the Whitehead torsion of $\lambda$ is in the image of $\pi_{0}(\alpha)$; hence we may glue a parametrized $h$-cobordism onto $p$ to obtain yet another factorization $f=p^{\prime} \circ \lambda^{\prime}$ where $\lambda^{\prime}$ is a simple homotopy equivalence in addition to 
being stably tangential (this uses Theorem 1.3). Thinking of $\lambda$ as a "thickening" in the sense of Wall [Wal66] and using the stable classification of these thickenings, it follows that $\lambda$ is stably homotopic to a homeomorphism. The virtue of this approach is that it produces an upper bound on the number of stabilizations needed whenever a concordance stable range for the fibers is known. See Theorem 5.10 for more details.

\section{Organization of the work}

The results concerning fibering questions are contained in section 5, The reader who is only interested in these can start reading there, jumping back when necessary. The section begins by stating and proving the main fibering theorems before giving several applications. Of special interest here is the "change of total space" problem, which avoids the more complicated Wall obstruction yet still allows interesting examples. Under smoothability assumptions, an upper bound on the number of stabilizations needed in the change of total space problem will be given. After that, a spectral sequence analysis of the Wall obstruction will lead to a re-interpretation of the results of ChapmanFerry in terms of our general obstruction theory. Finally the obstruction defined here will be related to the ones defined by Farrell-Lück-Steimle.

The first two sections of this text are devoted to the definition and properties of the parametrized Whitehead torsion map. It is built upon the works of Dwyer-Weiss-Williams on the parametrized A-theory characteristic [DWW03]. However, this characteristic is defined for a single bundle, whereas we want to get a map on the structure space. Therefore it will be necessary to consider a universal situation and use the parametrized characteristic there.

Therefore, in section 2, as well as defining structure spaces and giving elementary properties, a general method by Hughes-Taylor-Williams [HTW90] will be presented and slightly simplified, which interprets these structure spaces as spaces of lifts of certain classifying spaces.

The third section starts with the definition of the parametrized Whitehead torsion map. Afterwards some properties will be proved, including a composition rule, additivity (using results of Badzioch-Dorabiała [BD07]), and a theorem which can be interpreted as a product formula in a special case.

In section 4 the geometric assembly map is defined and Theorem 1.3 is proved. Similar results will be shown to hold on the level of Q-manifolds as well.

Finally, the appendix collects some technical results on fibrations, which are needed to make the classifying-space machinery work. 


\section{Acknowledgements}

I am indebted to my PhD advisor, Wolfgang Lück, for his constant advice, encouragement and generous support all through this project. Bruce Williams shared with me his interpretation of the obstructions from [FLS09] in terms of the A-theory characteristic and drew my interest to $Q$-manifold fibering, which was the starting point of this project. During a stay at Notre-Dame we spent a lot of time discussing about this work and he generously shared his knowlegde and ideas. I am especially grateful to Bruce Hughes for his hospitality during a stay at the University of Vanderbilt, his valuable suggestions, patience with my questions, and the numerous discussions we had. Among the many people I further wish to express my gratitude are: Arthur Bartels, Diarmuid Crowley, Jim Davis, Tom Farrell, Michael Joachim, Stacy Hoehn, Matthias Kreck and Tibor Macko for their interest, questions and suggestions; Silke Ahlers, Clara Löh, Adam Mole and Malte Röer for their help while proofreading the manuscript; and finally all the members of the topology group in Münster.

This work has been supported by the Graduiertenkolleg "Analytische Topologie und Metageometrie" of the Deutsche Forschungsgemeinschaft and the Leibniz-Preis of Wolfgang Lück. 


\section{Structure spaces on fibrations}

Given a space $X$, a manifold structure on $X$ is a homotopy equivalence $h: M \rightarrow$ $X$ from a compact manifold $M$ (possibly with boundary) to $X$. The structure space $\mathscr{S}_{n}(X)$ describes all the $n$-dimensional manifold structures on $X$. Instead of defining a topology on $\mathscr{S}_{n}(X)$, the standard procedure is to use a kind of "singular construction" to define $\mathscr{S}_{n}(X)$ as the geometric realization of a certain simplicial set.

Notice that (i) the object of interest are "honest" fibered structures (and not block structures), and that (ii) in contrast to surgery theory, there are no extra conditions on the boundary. For convenience all manifolds will be assumed to be topological, although all the constructions actually work in either of the categories of topological, differentiable, or Hilbert $Q$-manifolds.

The definition of $\mathscr{S}_{n}(X)$ has a straightforward generalization to the structure set of a fibration $p$, which will be defined in the first section. This is also the place where the homotopy invariance properties will be proved. In the second section, Corollary 2.8 will show that the structure space on a fibration $p$ with fiber $F$ is weakly homotopy equivalent to the space of lifts in the diagram

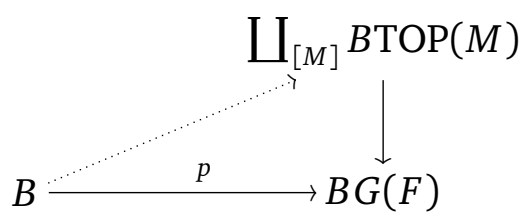

where the coproduct ranges over the isomorphism classes of compact $n$-manifolds homotopy equivalent to $F$. This result is well-known; however this section will present (and slightly simplify) the machinery of [HTW90] which can be used for a proof, as analogous results will be needed in a variety of similar situations later on.

We will always work in the category CGHaus of compactly generated Hausdorff spaces, and all constructions are to be taken in that category. 


\subsection{Definition of the structure space on a fibration}

Let $p: E \rightarrow B$ be a fibration over a paracompact space $B$. By an $n$-dimensional compact manifold structure on $p$, we mean a commutative diagram

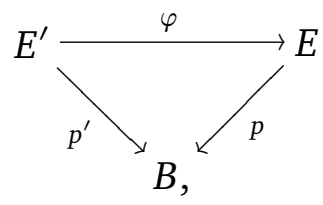

where

- $p^{\prime}$ is a bundle of $n$-dimensional compact (not necessary closed) manifolds (i.e. $p^{\prime}$ is the projection map of a fiber bundle with fibers $n$ dimensional compact topological manifolds), and

- $\varphi$ is a homotopy equivalence.

For set-theoretic reasons and to obtain good naturality properties, let us fix a set $\mathscr{U}$ of cardinality at least $2^{|\mathbb{R}|}$, and assume that $E^{\prime}$, as a set, is a subset of $B \times \mathscr{U}$, such that the inclusion $E^{\prime} \rightarrow B \times \mathscr{U}$ is a map over $B$ (with $B \times \mathscr{U} \rightarrow B$ being the projection onto the first factor). For future applications let us also fix a bijection $\mathscr{U} \times \mathscr{U} \rightarrow \mathscr{U}$.

With this proviso all $n$-dimensional compact manifold structures on $p$ form a set $\mathscr{S}_{n}(p)_{0}$. Given a pull-back square of fibrations

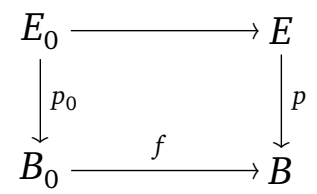

a compact manifold structure on $p$ induces a compact manifold structure on $p_{0}$ of the same dimension by restricting with $f$. More precisely, there is a unique topology on the set

$$
f^{*} E_{0}:=\left\{(b, e) \in B_{0} \times \mathscr{U} ;(f(b), e) \in E_{0}\right\}
$$

such that the obvious square becomes a topological pull-back diagram. Since the map $\varphi$ is actually a fiber homotopy equivalence, its restriction along $f$ still is a homotopy equivalence and thus defines a compact manifold structure on $E_{0}$.

This allows us to define a simplicial set $\mathscr{S}_{n}(p)$. by

$$
\mathscr{S}_{n}(p)_{k}:=\mathscr{S}_{n}\left(p \times \mathrm{id}_{\Delta^{k}}\right)_{0}
$$


such that the simplicial operations are induced by the restriction operation on the level of standard simplices.

Definition 2.1. The space of $n$-dimensional compact manifold structures on $p$ is the geometric realization

$$
\mathscr{S}_{n}(p):=\left|\mathscr{S}_{n}(p)\right| \cdot
$$

If $B$ is a point, we simply write $\mathscr{S}_{n}(E)$ for $\mathscr{S}_{n}(p)$.

Functoriality. Given a fiber homotopy equivalence $\psi: p^{\prime} \rightarrow p$ of fibrations over $B$, we clearly obtain obtain a simplicial map $\psi_{*}: \mathscr{S}_{n}\left(p^{\prime}\right)$. $\rightarrow \mathscr{S}_{n}(p)$. by composition, inducing a map on the structure spaces. On the other hand, given a pull-back square (1) of fibrations, the restriction operation leads to a $\operatorname{map} f^{*}: \mathscr{S}_{n}(p) \rightarrow \mathscr{S}_{n}\left(p_{0}\right)$.

Both the covariant and the contravariant operation are clearly functorial. Moreover they are homotopy invariant by the following two lemmas.

Lemma 2.2. If $\psi, \varphi: p^{\prime} \rightarrow p$ are fiber homotopy equivalences that are fiber homotopic, then $\psi_{*} \simeq \varphi_{*}: \mathscr{S}_{n}\left(p^{\prime}\right) \rightarrow \mathscr{S}_{n}(p)$.

Proof. Denote by $p^{\prime \prime}: E^{\prime} \times I \rightarrow E^{\prime} \rightarrow B$ the composite of the projection with $p^{\prime}$. The fiber homotopy between $\psi$ and $\varphi$ induces a fiber homotopy equivalence $H: p^{\prime \prime} \rightarrow p$ restricting to $\psi \amalg \varphi$ along $E^{\prime} \times \partial I$. So it is enough to show that there is a homotopy

$$
H: \mathscr{S}_{n}\left(p^{\prime}\right) \times I \rightarrow \mathscr{S}_{n}\left(p^{\prime \prime}\right)
$$

between the two maps induced by the front and the back inclusion.

$H$ is the geometric realization of a simplicial map, which on $k$-simplices is defined as follows: The pair $(y, x)$, with $y: E^{\prime} \rightarrow E \times \Delta^{k}$ a homotopy equivalence over $B \times \Delta^{k}$, and $x: \Delta^{k} \rightarrow \Delta^{1}$, is mapped to $i \circ y$, with

$$
i: E \times \Delta^{k} \stackrel{\mathrm{id}_{E} \times \mathrm{id}_{\Delta^{k}} \times|x|}{\longrightarrow} E \times \Delta^{k} \times I .
$$

Lemma 2.3. (i) Let $i_{0}, i_{1}: B \rightarrow B \times I$ be the inclusions at 0 and 1. Then $i_{0}^{*}, i_{1}^{*}: \mathscr{S}_{n}\left(p \times \mathrm{id}_{I}\right) \rightarrow \mathscr{S}_{n}(p)$ are homotopic.

(ii) Let $f: B^{\prime} \rightarrow B$ be a homotopy equivalence. Then so is $f^{*}: \mathscr{S}_{n}(p) \rightarrow \mathscr{S}_{n}\left(p^{\prime}\right)$.

Proof. (i) For all simplicial sets $X$, with geometric realization $X$, there is an "evaluation map"

$$
\mathscr{S}_{n}\left(p \times \operatorname{id}_{X}\right) \times X \rightarrow \mathscr{S}_{n}(p)
$$


defined on $k$-simplices as follows: The pair $(y, x)$, with $y$ a homotopy equivalence $E^{\prime} \rightarrow E \times X \times \Delta^{k}$ over $B \times X \times \Delta^{k}$, and $x: \Delta^{k}$. $\rightarrow X$., is sent to $r^{*} y$, the pull-back of $y$ via

$$
r: B \times \Delta^{k} \stackrel{\mathrm{id}_{B} \times \mathrm{id}_{\Delta^{k}} \times|x|}{\longrightarrow} B \times \Delta^{k} \times X .
$$

Now the evaluation map with $X .=\Delta^{1}$. shows that the maps $i_{0}^{*}, i_{1}^{*}: B \rightarrow B \times I$ induced by the front and back inclusions are homotopic.

(ii) Let us first show that if $f^{*}$ is a homotopy equivalence and $g \simeq f$, then $g^{*}$ is also a homotopy equivalence. In fact, if $H: B^{\prime} \times I \rightarrow B$ is a homotopy between $f$ and $g$, choose a fiber homotopy equivalence

$$
\varphi: f^{*} p \times \mathrm{id}_{I} \rightarrow H^{*} p
$$

over $B^{\prime} \times I$ which is the identity over $0 \in I$. By (i) we have

$$
\begin{aligned}
g^{*} & =i_{1}^{*} H^{*} \simeq\left(\left.\varphi\right|_{1}\right)_{*} i_{1}^{*}\left(\varphi_{*}\right)^{-1} H^{*} \simeq\left(\left.\varphi\right|_{1}\right)_{*} i_{0}^{*}\left(\varphi_{*}\right)^{-1} H^{*} \simeq\left(\left.\varphi\right|_{1}\right)_{*} i_{0}^{*} H^{*} \\
& =\left(\left.\varphi\right|_{1}\right)_{*} f^{*}: \mathscr{S}_{N}(p) \rightarrow \mathscr{S}_{n}\left(g^{*} p\right)
\end{aligned}
$$

So, if $f^{*}$ is a homotopy equivalence, then so is $g^{*}$ since $\left(\left.\varphi\right|_{1}\right)_{*}$ a homotopy equivalence by Lemma 2.2 .

Therefore, if $f$ is a homotopy equivalence, and $h$ is a fiber homotopy inverse for $f$, then both $f^{*} h^{*}$ and $h^{*} f^{*}$ are homotopy equivalences, say with homotopy inverses $\alpha$ and $\beta$. Then $\beta h^{*}$ is a left homotopy inverse for $f^{*}$, and also a right homotopy inverse, since

$$
f^{*} \beta h^{*} \simeq\left(\alpha f^{*} h^{*}\right)\left(f^{*} \beta h^{*}\right)=\alpha f^{*}\left(h^{*} f^{*} \beta\right) h^{*} \simeq \alpha f^{*} h^{*} \simeq \text { id } .
$$

So $f^{*}$ is a homotopy equivalence.

\subsection{Structure spaces as spaces of lifts}

For two spaces $F$ and $B$, with $B$ paracompact, let $\operatorname{Bun}_{n}(B ; F)$ be the category where an object is a bundle $E \rightarrow B$ with fibers compact $n$-dimensional topological manifolds homotopy equivalent to $F$. Again we assume that $E$, as a set, is a subset of $B \times \mathscr{U}$ and the inclusion map $E \rightarrow B \times \mathscr{U}$ is a map over $B$. Morphisms in this category are to be isomorphisms of such bundles.

Denote by cpCW the category of compact CW spaces, with continuous maps. Then the rule $X \mapsto \operatorname{Bun}_{n}(B \times X ; F)$ defines a functor

$$
\operatorname{Bun}_{n}(B ; F): \mathbf{c p C W}^{o p} \rightarrow \text { cat, }
$$


to the category of small categories. By giving an explicit system of simplices in cpCW (i.e. an embedding of categories $\Delta \rightarrow$ cpCW such that [ $n$ ] maps to an $n$-simplex and a morphism $[m] \rightarrow[n]$ maps to the corresponding face or degeneracy map), we can precompose to get a simplicial small category (i.e. simplicial object in the category of small categories)

$$
\operatorname{Bun}_{n}(B ; F) .: \Delta \rightarrow \text { cpCW } \rightarrow \text { cat. }
$$

It is obvious that different choices of systems of simplices leads to naturally isomorphic simplicial small categories.

Similarly define $\operatorname{Fib}(B ; F)$ to be the category where an object is a (Hurewicz) fibration over $B$ with fibers homotopy equivalent to $F$. We also require that the total space of the fibration is a subset of $B \times \mathscr{U}$ such that the inclusion map is fiberwise over $B$. A morphism in $\operatorname{Fib}(B ; F)$ is to be a fiber homotopy equivalence. Again this gives rise to a functor $\mathbf{c p C W}^{o p} \rightarrow$ cat by the rule $X \mapsto$ $\operatorname{Fib}(B \times X ; F)$ and therefore to a simplicial category, by precomposing with the system of simplices. Denote by $\operatorname{Bun}_{n}(B ; F)$. resp. $\operatorname{Fib}(B ; F)$. the simplicial sets which are given by the zero-nerves of the respective simplicial categories. Since $B$ is supposed to be paracompact, any bundle over $B \times \Delta^{n}$ is a fibration, such that we obtain a natural transformation $\operatorname{Bun}_{n}(B ; F) . \rightarrow \operatorname{Fib}(B ; F)$.

The following construction will finally lead to a description of $\mathscr{S}_{n}(p)$ as a space of lifts.

Consider a functor $\mathbf{C}: \mathbf{c p C W}^{o p} \rightarrow$ cat. Here are three properties that such a functor may have. In fact all our examples of such functors will satisfy all of these properties. It is useful to think of an object of $\mathbf{C}(X)$ as an object "over" $X$ and the functoriality operation as a restriction.

Amalgamation property. For any push-out square

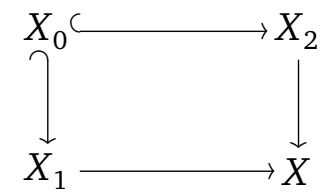

of compact CW spaces such that for $i=1,2$, the map $X_{0} \rightarrow X_{i}$ is the inclusion of a subcomplex, the square

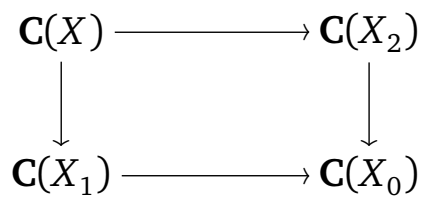

with inclusion-induced maps is a pull-back. 
Remark 2.4. (i) In comparison to [HTW90] this condition is slightly different. This difference does not affect the conclusions we are going to draw.

(ii) To verify that a commutative square

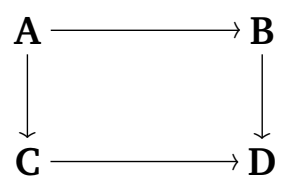

of categories is a pull-back, it is enough to verify the following two assertions:

(a) Given any two objects $b \in \mathbf{B}$ and $c \in \mathbf{C}$ projecting to the same element $d \in \mathbf{D}$, there exists a unique $a \in \mathbf{A}$ projecting to $b$ and $c$.

(b) Given any two morphisms $\beta$ in $\mathbf{B}$ and $\gamma$ in $\mathbf{C}$ projecting to the same morphism $\delta$ in $\mathbf{D}$, there exists a unique morphism $\alpha$ in A projecting to $\beta$ and $\gamma$.

Straightening Property. Denote by $p: \Delta^{k} \times I \rightarrow \Delta^{k}$ the projection and by $i: \Delta^{k} \rightarrow \Delta^{k} \times I$ the inclusion at 0 . For any object $E \in \mathbf{C}\left(\Delta^{k} \times I\right)$, there are to be morphisms

$$
F: E \rightarrow p^{*} i^{*} E \text { and } G: p^{*} i^{*} E \rightarrow E
$$

in $\mathbf{C}\left(\Delta^{k} \times I\right)$ which are both the identity map $i^{*} E \rightarrow i^{*} E$ upon restriction with $i$.

Fill-in property. Again denote by $p: \Delta^{k} \times I \rightarrow \Delta^{k}$ the projection. For any three objects $E_{0}, E_{1}, E \in \mathrm{C}\left(\Delta^{k}\right)$ and any two morphisms $\varphi_{i}: E_{i} \rightarrow E$, $i=0,1$, the following holds: There is an object $\bar{E}$ over $\Delta^{k} \times I$ and a morphism $\Phi: \bar{E} \rightarrow p^{*} E$ which restricts to $\varphi_{0}$ over 0 and to $\varphi_{1}$ over 1 .

Moreover, given two more objects $F_{0}, F_{1}$ over $\Delta^{k}$ together with morphisms $\psi_{i}: F_{i} \rightarrow E, i=0,1$, which agree with the data $\left(E_{0}, E_{1}, \varphi_{0}, \varphi_{1}\right)$ when restricted to a collection of faces of $\Delta^{k}$, there are extensions $(\bar{E}, \Phi)$ and $(\bar{F}, \Psi)$ of $\left(E_{0}, E_{1}, \varphi_{0}, \varphi_{1}\right)$ and $\left(F_{0}, F_{1}, \psi_{0}, \psi_{1}\right)$ that agree when restricted to the same collection $\times I$.

We again restrict our functor $\mathbf{C}: \mathbf{c p C W}^{o p} \rightarrow$ cat to a simplicial category C. : $\Delta^{o p} \rightarrow$ cat. Any simplicial small category $\mathbf{C}$. gives rise to three simplicial sets: 
- The 0-nerve $C$. $:=N_{0} \mathbf{C}$,

- The (diagonal of the) bisimplicial set N.C., and

- For each object $c \in \mathrm{C}_{0}$, the (diagonal of the) nerve $N$. End(c). of the simplicial monoid End $(c)$. The $k$-simplices of $\operatorname{End}(c)$. are just the endomorphisms of $c \in \mathbf{C}_{k}$. (The object $c$ is understood to be lifted to $\mathbf{C}_{k}$ via the degeneracy operation.)

If the original functor $\mathbf{C}$ satisfies the Amalgamation, Straightening, and Fill-in properties, then the following holds (see [HTW90, §§ 7 and 8]):

(i) All simplicial sets $N_{k}$ C. are Kan.

(ii) The natural inclusion $C=N_{0} \mathrm{C} \rightarrow N$. C is a homotopy equivalence.

(iii) The natural inclusion $\operatorname{End}(c)_{k} \rightarrow \mathbf{C}_{k}$, for all objects $c \in \mathbf{C}_{0}$, gives rise to a homotopy equivalence

$$
\coprod_{[c] \in \pi_{0} C .} N . \operatorname{End}(c) . \rightarrow N . \text { C. }
$$

(iv) Suppose that $\mathbf{D}: \mathbf{c p C W}^{o p} \rightarrow$ cat is another functor and that $\mathbf{f}: \mathbf{D}$. $\rightarrow \mathbf{C}$. is a natural transformation between the associated simplicial categories. For $c \in \mathbf{C}_{0}$, the comma categories $\mathbf{f}_{k} / c$ define a simplicial small category f. $/ c$ whose zero-nerve fits into a homotopy fibration sequence

$$
N_{0} \mathbf{f} . / c \rightarrow D . \stackrel{f .}{\rightarrow} C .
$$

(homotopy fiber over the point $c \in C_{0}$ ).

The following has been proven in a slightly different form in [HTW90, §7].

Lemma 2.5. Both functors $\operatorname{Bun}_{n}(B ; F)$ and $\mathbf{F i b}(B ; F)$ satisfy the Amalgamation, Straightening, and Fill-in properties provided $B$ is metrizable and ULC.

Recall that a space $B$ is ULC (or locally equiconnected) if there is a neighborhood $U \subset B \times B$ of the diagonal and a homotopy

$$
H: U \times I \rightarrow B
$$

between the first and the second projection which is relative to the diagonal. For example, if $B$ is a metrizable ANR (e.g., a locally finite CW complex), then it is also ULC.

Proof. For bundles, the Amalgamation property is classical, and so is the Straightening property. Fill-ins are given by mapping cylinders. (Strictly 
speaking, given a map $\varphi: E \rightarrow E^{\prime}$ over $B$, think of its mapping cylinder as a subset $E \times[0,1) \cup E^{\prime} \times\{1\}$ of $B \times I \times \mathscr{U}$, endowed with the suitable topology.)

As for fibrations, see A.12 for the Amalgamation property. Straightening for fibrations follows from homotopy lifting. The existence of fill-ins for fibrations is proven in Proposition A.18.

Corollary 2.6. (i) For any fibration $p: E \rightarrow B$ which is an object of $\mathbf{F i b}(B ; F)$ over a metrizable ULC base space, there is a simplicial homotopy equivalence

$$
\mathscr{S}_{n}(p) . \rightarrow \operatorname{hofib}_{p}\left(\operatorname{Bun}_{n}(B ; F) . \rightarrow \operatorname{Fib}(B ; F) .\right)
$$

which is natural in $B$.

(ii) We have

$$
\operatorname{Bun}_{n}(* ; F) . \simeq \coprod_{[M]} B \mathrm{TOP}(M) ., \quad \operatorname{Fib}(* ; F) . \simeq B G(F)
$$

where $\operatorname{TOP}(M)$. resp. $G(F)$. denotes the simplicial monoid of self-homeomorphisms resp. self-homotopy equivalences, and the coproduct ranges over all isomorphism classes of compact n-manifolds homotopy equivalent to $F$.

Now we are going to show that we have obtained suitable models for classifying spaces.

Lemma 2.7. Let $X$. be a locally finite simplicial set.

(i) There are natural simplicial isomorphisms

$$
\begin{aligned}
\operatorname{Bun}_{n}(|X .| ; F) & \cong \operatorname{map} .\left(X ., \operatorname{Bun}_{n}(* ; F) .\right) \\
\operatorname{Fib}(|X .| ; F) . & \cong \operatorname{map} .(X ., \operatorname{Fib}(* ; F) .)
\end{aligned}
$$

(ii) For any fibration $p: E \rightarrow B$, with $B$ the geometric realization of a locally finite simplicial set $B$., there is a natural simplicial isomorphism

$$
\operatorname{hofib}_{p}\left(\operatorname{Bun}_{n}(B ; F) . \rightarrow \operatorname{Fib}(B ; F) .\right) \rightarrow \operatorname{Lift}\left(\begin{array}{ccc} 
& \operatorname{Bun}_{n}(* ; F) . \\
& p & \operatorname{Fib}(* ; F) .
\end{array}\right)
$$


Notation. Here and in the following, when referring to a space of lifts, we will always implicitly assume that the vertical map has been converted into a fibration (Kan fibration for simplicial sets or Hurewicz fibration for topological spaces).

Proof. (i) We only treat the case of bundles; the other case is completely analogous. We only need to give a natural bijection

$$
D: \operatorname{Bun}_{n}(|X .| \times Y ; F)_{0} \rightarrow \operatorname{map}_{0}\left(X . \operatorname{Bun}_{n}(Y ; F) .\right)
$$

on the level of 0-simplices.

Let $q: E \rightarrow|X| \times$.$Y be a 0$-simplex in the left hand side. We need to associate to it a simplicial map $X$. $\rightarrow \operatorname{Bun}_{n}(Y ; F)$. Therefore let $\sigma$ be an $l$-simplex of $X$., represented by a simplicial map $\sigma: \Delta^{l} \rightarrow X$. The pull-back $\left(|\sigma| \times \mathrm{id}_{Y}\right)^{*} q$ is then a bundle over $\Delta^{l} \times Y$ which defines an $l$-simplex in $\operatorname{Bun}_{n}(Y ; F)$.

Here is a description of the inverse $D^{\prime}$ of $D$. Let $\phi .: X$. $\rightarrow \operatorname{Bun}_{n}(Y ; F)$. be a simplicial map. For a nondegenerate $k$-simplex $\tau$ of $\operatorname{Bun}_{n}(Y ; F)$., denote by $E(\tau)$ the total space of the corresponding bundle over $B(\tau)=Y \times \Delta^{k}$.

Now define $E \rightarrow|X| \times$.$Y to be the bundle$

$$
\bigcup_{\sigma} E(\phi(\sigma)) \rightarrow \bigcup_{\sigma} B(\phi(\sigma))
$$

with $\sigma$ ranging over the nondegenerate simplices of $X$.. If $X$. is finite, then this is a bundle by the Amalgamation property. Otherwise use the fact that there is an open cover of $|X$.$| such that each element of the cover is contained in a$ finite simplicial subset.

The total space is canonically a subset of $|X| \times . Y \times \mathscr{U}$, so the bundle is really a zero simplex in $\operatorname{Bun}_{n}(|X| \times Y ; F$.$) . and the map D^{\prime}$ is a strict inverse of D.

(ii) follows from (i).

Denote by $\operatorname{Fib}(B ; F)$ the geometric realization of $\operatorname{Fib}(B ; F)$, and define $\operatorname{Bun}_{n}(B ; F)$ similarly.

Corollary 2.8. If $B$ is a locally finite ordered simplicial complex, then there is a natural weak homotopy equivalence

$$
\mathscr{S}_{n}(p) \rightarrow \operatorname{Lift}\left(\begin{array}{ccc} 
& \operatorname{Bun}_{n}(* ; F) \\
& & \downarrow \\
B & \stackrel{p}{\longrightarrow} & \operatorname{Fib}(* ; F)
\end{array}\right) .
$$


Remark 2.9. There is still a weak homotopy equivalence, well-defined up to homotopy, if $B$ is homotopy equivalent to a locally finite ordered simplicial complex, as both domain and target are homotopy invariant.

Proof of Corollary. This follows from our simplicial results obtained so far after passage to geometric realization. Therefore we are left to show that the map induced by geometric realization

$$
\begin{aligned}
& \operatorname{hofib}_{p}\left(\operatorname{map} .\left(B ., \operatorname{Bun}_{n}(* ; F) .\right) \rightarrow \operatorname{map} .(B ., \operatorname{Fib}(* ; F) .)\right) \\
& \rightarrow \operatorname{hofib}_{p}\left(\operatorname{map}\left(B, \operatorname{Bun}_{n}(* ; F)\right) \rightarrow \operatorname{map}(B, \operatorname{Fib}(* ; F))\right)
\end{aligned}
$$

is a weak equivalence.

For any Kan complex $Y$., the geometric realization map map.(X., $Y$.) $\rightarrow$ $\operatorname{map}(X, Y)$ is a weak homotopy equivalence. Moreover, since we assumed $\operatorname{Bun}_{n}(* ; F)$. $\rightarrow \mathrm{Fib}(* ; F)$. to be converted to a Kan fibration, its geometric realization is a Serre fibration, by a well-known theorem of Quillen, and the inclusion of its fiber into the Hurewicz fiber is a weak homotopy equivalence.

\subsection{Universal "bundles"}

Using the construction in the proof of Lemma 2.7, we can associate to the identity map $\operatorname{Bun}_{n}(* ; F)$. $\rightarrow \operatorname{Bun}_{n}(* ; F)$. a map

$$
\tilde{\mathscr{E}}_{n} \rightarrow \tilde{\mathscr{B}}:=\left|\operatorname{Bun}_{n}(* ; F) .\right|
$$

which, by Lemma 2.7 , is a bundle over every locally finite subcomplex of $\tilde{\mathscr{B}}$.

Similarly, we obtain a map

$$
\mathscr{E} \rightarrow \mathscr{B}:=|\operatorname{Fib}(* ; F)|
$$

which is a fibration over every locally finite subcomplex of $\mathscr{B}$.

We will speak of both maps as the universal "bundles", although we didn't prove any result that they are actually bundles or fibrations. In fact we will see that the stated property is just good enough for our purposes. 


\section{The parametrized Whitehead torsion}

The classical Whitehead torsion defines a map

$$
\tau: \pi_{0} \mathscr{S}_{n}(M) \rightarrow \mathrm{Wh}\left(\pi_{1} M\right), \quad[f: N \rightarrow M] \mapsto \tau(f)
$$

for a compact topological manifold $M$, using the extension of classical simple homotopy theory to compact ANRs (or using Chapman's theorem of homeomorphism invariance of the Whitehead torsion together with results of KirbySiebenmann on topological manifolds).

In this section we will define a "parametrized Whitehead torsion"

$$
\tau: \mathscr{S}_{n}(p) \rightarrow \Gamma\left(\begin{array}{c}
\Omega \mathrm{Wh}_{B}(E) \\
\downarrow \\
B
\end{array}\right)
$$

whenever $p: E \rightarrow B$ is a bundle of compact topological manifolds. Here the right-hand side is the space of sections of a fibration over $B$ which is obtained from $p$ by applying $\Omega$ Wh fiberwise, as defined by [DWW03] (see below). The symbol Wh denotes the connective topological Whitehead functor as defined by Waldhausen. In the case where $B$ is a point, the parametrized Whitehead torsion reduces to

$$
\tau: \mathscr{S}_{n}(E) \rightarrow \Omega \mathrm{Wh}(E)
$$

whose induced map on path components will be identified to the classical Whitehead torsion (Section 3.8).

The definition of the parametrized torsion can be outlined as follows: Given a fibration $p: E \rightarrow B$ satisfying a finiteness condition on the fibers, Dwyer-Weiss-Williams define a "parametrized $A$-theory characteristic"

$$
\chi(p) \in \Gamma\left(\begin{array}{c}
A_{B}(E) \\
\downarrow \\
B
\end{array}\right) .
$$

If $p$ is actually a fiber bundle of compact topological manifolds, then it has a canonical refinement over the fiberwise assembly map

$$
\Gamma\left(\begin{array}{c}
A_{B}^{\%}(E) \\
\downarrow \\
B
\end{array}\right) \rightarrow \Gamma\left(\begin{array}{c}
A_{B}(E) \\
\downarrow \\
B
\end{array}\right) .
$$


Letting $\mathscr{B}$ be the base space of the universal fibration with fiber $F$ and $\tilde{\mathscr{B}}$ the base space of the universal bundle with fibers compact $n$-manifolds homotopy equivalent to $F$, the results of Dwyer-Weiss-Williams therefore yield a diagram

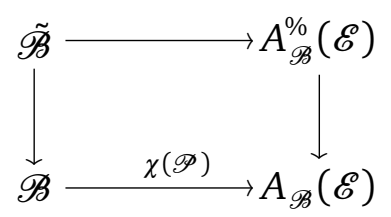

Hoehn [Hoe09] used this diagram to produce a map

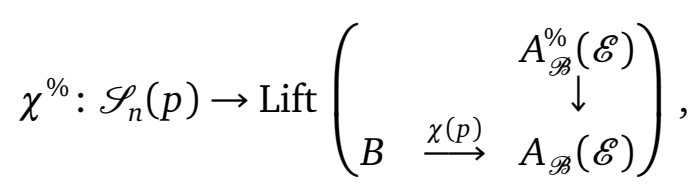

by thinking of $\mathscr{S}_{n}(p)$ as a space of lifts. The map $\chi^{\%}$ will be called parametrized excisive characteristic by us.

The transition from the parametrized excisive characteristic to the parametrized torsion requires an analysis of the loop space structure on the range of $\chi^{\%}$. In fact, up to weak homotopy equivalence, there is an "addition" map

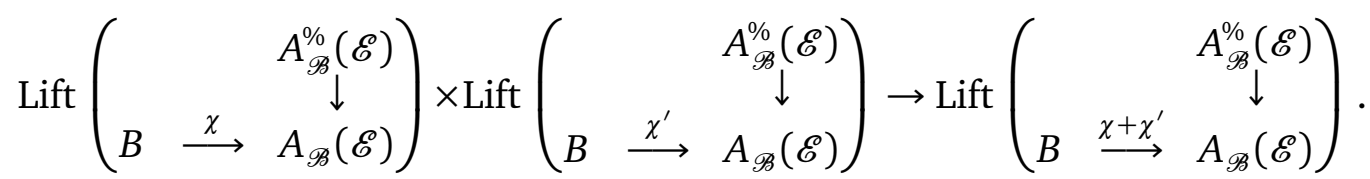

So, if $p$ is a bundle of compact $n$-manifolds, we can consider the the difference between $\chi^{\%}$ and the constant map $\chi^{\%}(p)$. Using that

$$
\operatorname{Lift}\left(\begin{array}{ccc} 
& A_{\mathscr{B}}^{\%}(\mathscr{E}) \\
& & \downarrow \\
B & \stackrel{0}{\longrightarrow} & A_{\mathscr{B}}(\mathscr{E})
\end{array}\right) \simeq \Gamma\left(\begin{array}{c}
\Omega \mathrm{Wh}_{B}(E) \\
\downarrow \\
B
\end{array}\right)
$$

we obtain the parametrized torsion map

Just as the classical torsion is invariant under stabilization $M \mapsto M \times I$, the same is true for the parametrized version. The stabilized torsion map, defined on the stable structure space, will be important for us since we will see in section 4 that its homotopy fiber can be explicitly described.

The first subsection reviews the construction of the parametrized A-theory characteristic as defined by Dwyer-Weiss-Williams [DWW03]. After defining the parametrized torsion and its stabilized version, we will establish certain 
properties of the parametrized torsion map, such as additivity, composition rule, and a particular case of a product formula. They will be of central importance in the following sections.

Finally, in section 3.7, we relate the parametrized torsion to Waldhausen's map, which is a homotopy equivalence from a space of stable $h$-cobordisms on $M$ to $\Omega W h(M)$ (section 3.7). This allows to identify $\pi_{0}$ of the section space appearing in the map $\tau$ with isomorphism classes of "stable parametrized $h$ cobordisms over $p$ ".

\subsection{The parametrized A-theory characteristic}

Let $p: E \rightarrow B$ be a fibration over a space which is the geometric realization of a simplicial set $B$., such that the fibers are homotopy finitely dominated. Given such a fibration, one can associate the parametrized $A$-theory characteristic

$$
\chi(p) \in \operatorname{holim}_{\sigma \in \operatorname{simp} B .} A\left(E_{\sigma}\right) .
$$

Here $\operatorname{simp} B$ is the category of simplices of the simplicial set $B$. and $E_{\sigma}=|\sigma|^{*} E$, the pull-back of $E$ to a bundle over the simplex $|\sigma|$. See the first remark below for a review of the definition of $\chi(p)$.

The homotopy limit can be understood, up to homotopy, as a space of sections of a fibration over $B$ which is obtained from $p$ by applying the $A$ theory functor fiberwise:

$$
\underset{\sigma \in \operatorname{simp} B .}{\operatorname{holim}} A\left(E_{\sigma}\right) \simeq \Gamma\left(\begin{array}{c}
A_{B}(E) \\
\downarrow \\
B
\end{array}\right) .
$$

See the second remark below for this identification.

Background on characteristics. Denote by cat the category of small categories. Given a small category $\mathscr{C}$ and a functor $F: \mathscr{C} \rightarrow$ cat, a characteristic for $F$ is a natural transformation

$$
\chi: \mathscr{C} / ? \rightarrow F
$$

where $\mathscr{C} / c$ is the over category where an object is a morphism $d \rightarrow c$ in $\mathscr{C}$ and a morphism is a commutative triangle.

Unraveling the definitions, we see that $\chi$ is given by the following data:

(i) For each object $c$ of $\mathscr{C}$, a "characteristic object" $c^{!} \in F(c)$, which corresponds to the image of the identity morphism on $c$ under the functor $\chi(c)$, and 
(ii) For each morphism $\varphi: c \rightarrow d$ in $\mathscr{C}$, a morphism $\varphi^{!}: \varphi_{*}\left(c^{!}\right) \rightarrow d^{!}$, satisfying the cocycle condition $(e f)^{!}=e^{!} \circ e_{*}\left(f^{!}\right)$.

For us, there are basically two ways how such characteristics occur:

(i) Let $f$ be a space-valued functor on $\mathscr{C}$ such that all objects are mapped to homotopy finitely dominated spaces, and all morphisms are mapped to homotopy equivalences. If we denote by $\mathscr{R}^{f d}(X)$ the Waldhausen category of homotopy finitely dominated spaces over $X$, we obtain a characteristic for the functor $\mathscr{R}^{f d} \circ f$, with characteristic object $c^{!}=$ $f(c) \times S^{0}$, considered as a retractive space over $f(c)$.

(ii) If $f$ maps all objects even to compact ENRs, and maps all morphisms to cell-like maps between these, then we obtain a characteristic for a specific functor $\mathscr{R}^{\%} \circ f$, with $\mathscr{R}^{\%}(X)$ defined below. It has the feature that $\left|\mathscr{R}^{\%}(X)\right|$ maps to $A^{\%}(X)$.

To get back to the $A$-theory characteristic, let $\mathscr{C}=\operatorname{simp} B$. be the category of simplices of the simplicial set $B$, and let $f(\sigma)=E_{\sigma}:=|\sigma|^{*} E$. The prodedure just described yields a characteristic on $F=\mathscr{R}^{f d} \circ f$.

Now let $G: \mathscr{C} \rightarrow$ CGHaus be a functor. Then a characteristic for $G$ is defined to be a natural transformation

$$
\chi:|\mathscr{C} / ?| \rightarrow G
$$

Obviously the geometric realization of a characteristic on $F$ as above defines a characteristic on $|F|$. In our example above, notice that there is a natural map

$$
\left|\mathscr{R}^{f d}\left(E_{\sigma}\right)\right| \rightarrow K\left(\mathscr{R}^{f d}\left(E_{\sigma}\right)\right)=A\left(E_{\sigma}\right)
$$

reminiscent of the group completion. Hence, by composition of natural transformations, we also obtain a characteristic for the functor

$$
\sigma \mapsto A\left(E_{\sigma}\right)
$$

The space of all characteristics for $G$, endowed with its canonical topology, is by definition the homotopy $\operatorname{limit}$ holim $G$. In summary, the characteristic on the functor $F: \operatorname{simp} B . \rightarrow$ cat defined above produces an element

$$
\chi(p) \in \operatorname{holim}_{\sigma \in \operatorname{simp} B} A\left(E_{\sigma}\right) .
$$

Background on homotopy limits and section spaces. For a functor $F$ from a small category $\mathscr{C}$ to spaces, working with the standard model for the homotopy 
limit, a point $\chi \in \operatorname{holim} F$ is just a natural transformation $\mid \mathscr{C} /$ ? $\mid \rightarrow F$. Such a natural transformation induces a lift

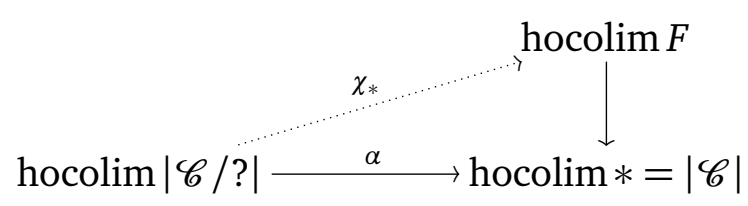

of the canonical projection $\alpha$. The homotopy equivalence $\alpha$ induces a homotopy equivalence

$$
\alpha^{*}: \Gamma\left(\begin{array}{c}
\operatorname{hocolim} F \\
\downarrow \\
|\mathscr{C}|
\end{array}\right) \stackrel{\simeq}{\rightarrow} \operatorname{Lift}\left(\begin{array}{ccc} 
& & \operatorname{hocolim} F \\
\operatorname{hocolim}|\mathscr{C} / ?| & \longrightarrow & |\mathscr{C}|
\end{array}\right) .
$$

(Recall that in our notation, all vertical maps have been converted into fibrations.)

If $F$ sends all morphisms to homotopy equivalences, we obtain a zigzag of weak homotopy equivalences

$$
\operatorname{holim} F \simeq \Gamma\left(\begin{array}{c}
\operatorname{hocolim} F \\
\downarrow \\
|\mathscr{C}|
\end{array}\right)
$$

In our case, $\mathscr{C}=\operatorname{simp} B$., so $|\mathscr{C}| \simeq B$ by Kan's last vertex map. The functor $F$ sends a simplex $\sigma \in \operatorname{simp} B$. to the space $E_{\sigma}:=|\sigma|^{*} E$, the total space of the pull-back of $E$ over $\sigma$. By definition, the map $A_{B}(E) \rightarrow B$ is the fibration associated with the composite hocolim $F \rightarrow|\mathscr{C}| \rightarrow B$.

If the fibration $p$ happens to be a bundle with fibers compact (not necessarily closed) topological manifolds, Dwyer-Weiss-Williams also define an excisive characteristic. Informally, it can be understood as a refinement of $\chi(p)$ in the sense that it defines, up to homotopy, and element in

$$
\chi^{\%}(p) \in \operatorname{Lift}\left(\begin{array}{ccc} 
& & A_{B}^{\%}(E) \\
& & \downarrow \\
B & \stackrel{\chi(p)}{\longrightarrow} & A_{B}(E)
\end{array}\right)
$$

where the vertical map is the fiberwise assembly map $\alpha$. Thus it defines a section of the fibration over $B$ obtained by applying the functor $A^{\%}$ fiberwise, together with a path from $\alpha \chi^{\%}(p)$ to $\chi(p)$. In the homotopy limit language 
we obtain (up to homotopy) an element in the homotopy fiber over $\chi(p)$ of the map

$$
\operatorname{holim}_{\sigma \in \operatorname{simp} B .} A^{\%}\left(E_{\sigma}\right) \rightarrow \operatorname{holim}_{\sigma \in \operatorname{sim} B} A\left(E_{\sigma}\right)
$$

Formally, the excisive characteristic is an element in another homotopy limit space whose definition we will not repeat at this place. For more details, consult subsection 3.4 .

Naturality. If $f: B^{\prime} \rightarrow B$. is a simplicial map, then we have the following naturality properties:

Lemma 3.1. (i) If $p: E \rightarrow B$ is a fibration as above, then under the restriction map

$$
\operatorname{holim}_{\sigma \in \operatorname{sim} B .} A\left(E_{\sigma}\right) \rightarrow \operatorname{holim}_{\sigma \in \operatorname{simp} B^{\prime}} A\left(E_{\sigma}\right)
$$

the A-theory characteristic $\chi(p)$ maps to the A-theory characteristic of the retriction $p^{\prime}$ of $p$ along $f$.

(ii) If $p$ is a bundle of compact topological manifolds, then there is a canonical homotopy class of paths between $\chi^{\%}\left(p^{\prime}\right)$ and the image of $\chi^{\%}(p)$ under the restriction map

$$
\operatorname{hofib}_{\chi(p)}\left(\begin{array}{c}
\operatorname{holim}_{\sigma \in \operatorname{simp} B .} A^{\%}\left(E_{\sigma}\right) \\
\downarrow \\
\operatorname{holim}_{\sigma \in \operatorname{simp} B} A\left(E_{\sigma}\right)
\end{array}\right) \rightarrow \operatorname{hofib}_{\chi\left(p^{\prime}\right)}\left(\begin{array}{c}
\operatorname{holim}_{\sigma \in \operatorname{simp} B^{\prime}} A^{\%}\left(E_{\sigma}\right) \\
\downarrow \\
\operatorname{holim}_{\sigma \in \operatorname{simp} B^{\prime}} A\left(E_{\sigma}\right)
\end{array}\right)
$$

Remark 3.2. The models for the A-theory spaces that we are going to use are the following:

$$
A(X)=K\left(\mathscr{R}^{f d}(X)\right),
$$

thus the $K$-theory of the Waldhausen category of homotopy finitely dominated spaces over $X$, and

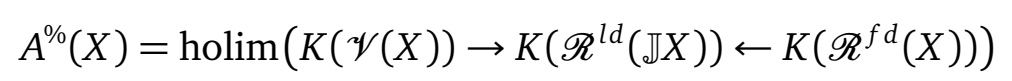

as in [DWW03, section 8]. See p. 58 for the definition of the excisive characteristic in this model. It does not have the best possible naturality properties in that it is not natural under localizations, but it has the advantage that the assembly map is easily defined as the projection from the homotopy limit to $K\left(\mathscr{R}^{f d}(X)\right)$.

Following [BD07], we denote by $\mathscr{R}^{\%}(X)$ the pull-back of

$$
\mathscr{V}(X) \rightarrow \mathscr{R}^{l d}(\mathbb{J} X) \leftarrow \mathscr{R}^{f d}(X),
$$


thus obtaining a canonical map $K\left(\mathscr{R}^{\%}(X)\right) \rightarrow A^{\%}(X)$. Hence any characteristic for the functor $X \mapsto \mathscr{R}^{\%}(X)$ produces an $A^{\%}$-valued characteristic. The excisive characteristic that we will use is defined this way.

\subsection{Definition}

\section{The parametrized excisive characteristic}

Recall from section 2 that $\mathscr{B}:=|\operatorname{Fib}(* ; F)$.$| and \tilde{\mathscr{B}}:=\left|\operatorname{Bun}_{n}(* ; F).\right|$ carry universal "bundles". They have the property that the restriction of the "bundles" over every locally finite subcomplex are fibrations resp. bundles. This is good enough to define parametrized characteristics, which only make use of the restrictions over simplices.

Choose a representative

$$
\chi(\mathscr{P}) \in \Gamma\left(\begin{array}{c}
A_{\mathscr{B}}(\mathscr{E}) \\
\downarrow \\
\mathscr{B}
\end{array}\right)
$$

of the parametrized $A$-theory characteristic of the universal "bundle" over $\mathscr{B}$. We obtain a commutative diagram as follows

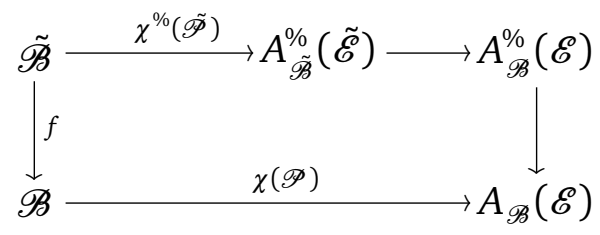

by the following lemma, applied to the forgetful map $f: \tilde{\mathscr{B}} \rightarrow \mathscr{B}$ which considers a bundle as a fibration.

Lemma 3.3. Given a simplicial map $f .: B$. $\rightarrow \mathscr{B}$. together with an element $\chi \in$ holim $_{\sigma \in \operatorname{simp} B} A\left(E_{\sigma}\right)$, there is a zigzag of weak homotopy equivalences

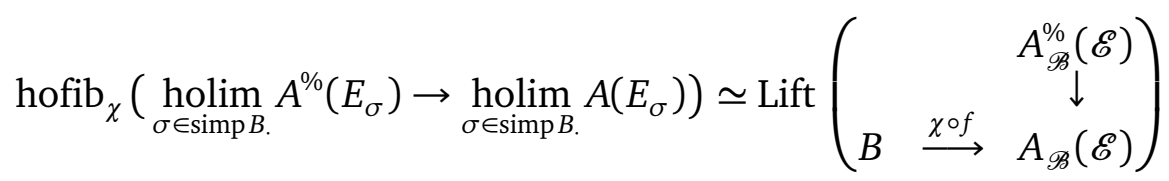

which is natural in $B$. 
Proof. The square

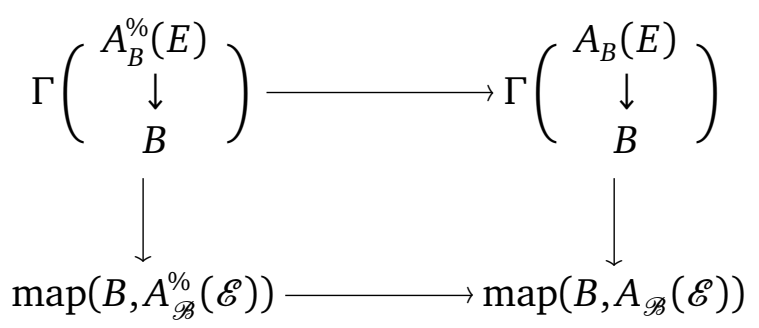

is a weak homotopy pull-back. Now recall that the section spaces in the upper line are weakly homotopy equivalent to the corresponding homotopy limits.

Composition with diagram (1) induces a map

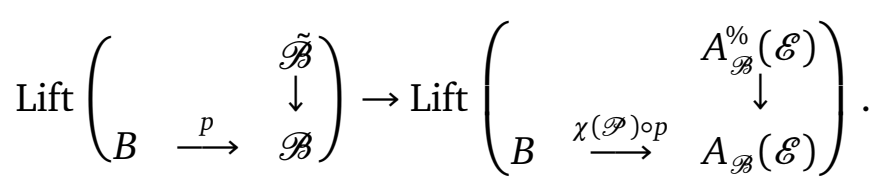

Notation. From now on, suppose that one choice for $\chi(\mathscr{P})$ and for the diagram (1) has been made. The notation $\chi(p)$ will, by abuse of notation, also denote the map $\chi(\mathscr{P}) \circ p$.

Definition 3.4. Let $p: E \rightarrow B$ be a fibration which is an object of $\operatorname{Fib}(B ; F)$ with a fiber $F$ that is homotopy finitely dominated. Suppose that the $B$ is homotopy equivalent to a locally finite simplicial complex. The parametrized excisive characteristic of $p$ is the composite

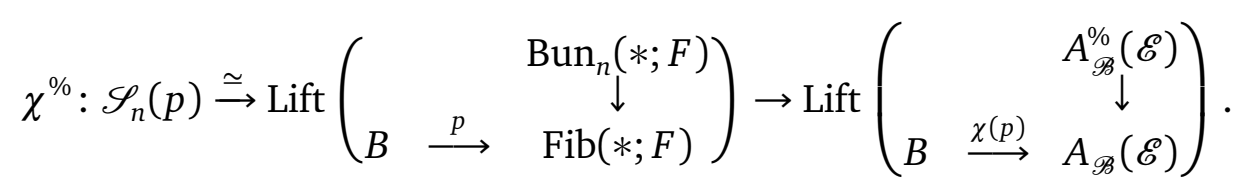

\section{Loop space structure and the parametrized torsion}

Notice that in Lemma 3.3, in the right-hand side the loop space structure is not visible any more, while it is present in the left-hand side. Hence, also the range of the parametrized excisive characteristic carries such a structure. For example, up to weak homotopy equivalence, there is a "difference map"

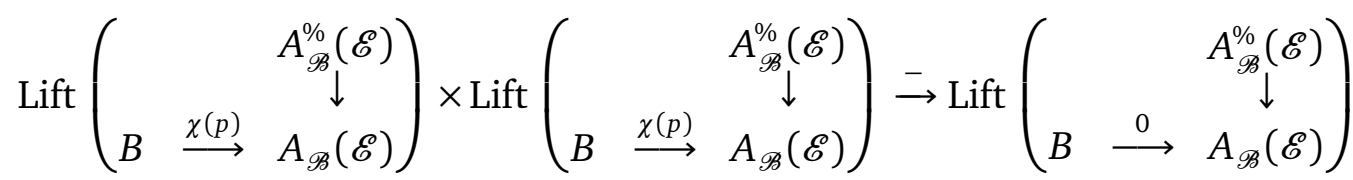


where we may identify the target with holim $\operatorname{lesimp}_{B} \Omega \mathrm{Wh}\left(E_{\sigma}\right)$ and hence with a section space.

Definition 3.5. $\quad$ (i) The parametrized Whitehead torsion map

$$
\tau: \mathscr{S}_{n}(p) \times \mathscr{S}_{n}(p) \rightarrow \Gamma\left(\begin{array}{c}
\Omega \mathrm{Wh}_{B}(E) \\
\downarrow \\
B
\end{array}\right)
$$

is given by $\tau(?, ? ?)=\chi^{\%}(?)-\chi^{\%}($ ??).

(ii) If $p$ is itself an object of $\operatorname{Bun}_{n}(B ; F)$, it defines a canonical element id $\in$ $\mathscr{S}_{n}(p)$. In this case, we simply write

$$
\tau=\tau(\cdot, \mathrm{id}): \mathscr{S}_{n}(p) \rightarrow \Gamma\left(\begin{array}{c}
\Omega \mathrm{Wh}_{B}(E) \\
\downarrow \\
B
\end{array}\right)
$$

and also refer to it as the parametrized Whitehead torsion map.

Just as the excisive characteristic, the torsion is well-defined up to homotopy.

\section{Naturality properties}

The excisive characteristic is natural with respect to fiber homotopy equivalences in the following sense: Let $p: E \rightarrow B$ and $p^{\prime}: E^{\prime} \rightarrow B$ be fibrations for which the excisive characteristic is defined, and let $\varphi: p \rightarrow p^{\prime}$ be a fiber homotopy equivalence. Define a map

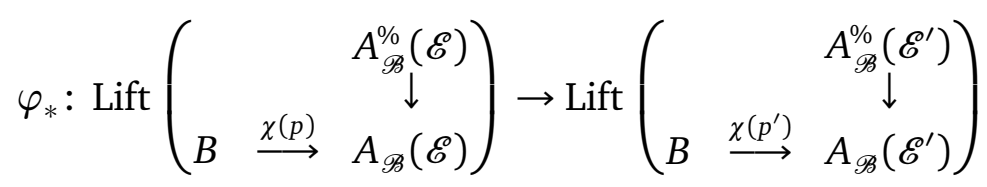

as follows: The fiber homotopy equivalence $\varphi$ induces a homotopy between the maps $p, p^{\prime}: B \rightarrow \mathscr{B}$ and therefore a homotopy between $\chi(p), \chi\left(p^{\prime}\right): B \rightarrow$ $A_{\mathscr{B}}(\mathscr{E})$. The map $\varphi_{*}$ is induced by standard fiber transport along this homotopy.

Then we have $\varphi_{*} \circ \chi^{\%} \simeq \chi^{\%} \circ \varphi_{*}$. This follows from the definitions.

Moreover, the parametrized torsion is compatible with pullbacks. More precisely: 
Remark 3.6. For $f: B^{\prime} \rightarrow B$, the following square with obvious maps is commutative up to homotopy:

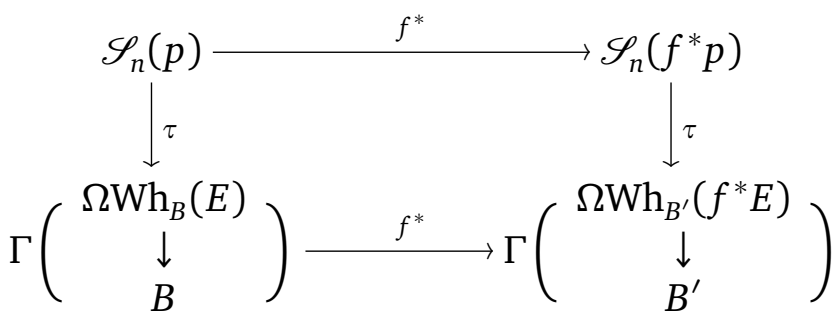

\section{A compatibility result}

To formulate our next result, let us rewrite the first construction in more formal terms. As we pointed out, composition defines a map

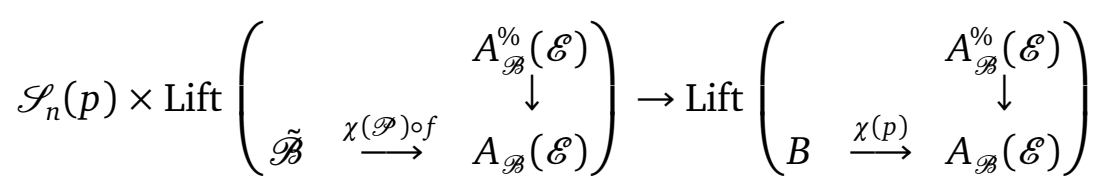

(using the description of the structure space as a space of lifts). It is adjoint to

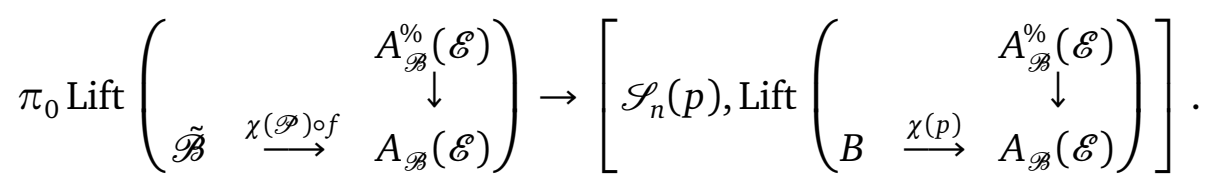

Now the constructions of Dwyer-Weiss-Williams produce an element in the left hand side of this adjoint, by Lemma 3.3. The image of this element in the right-hand side is the parametrized excisive characteristic as defined above.

Lemma 3.7. The map (3) preserves the loop space structure induced by Lemma 3.3

This result shows that whenever the Dwyer-Weiss-Williams characteristics of certain bundles decompose as a sum of other characteristics, the same is true for the parametrized excisive characteristic (and hence for the parametrized Whitehead torsion). This will be important in the proof of the Additivity theorem down below.

The remainder of this subsection will be devoted to the proof of this Lemma. While the idea of proof is simple - define the map directly on the level of homotopy limits using precomposition alias restriction - the proof is 
technically more difficult due to the replacement of the simplicial set $B$. by its category of simplices.

For example, we would like to define a map

$$
\operatorname{holim}_{\sigma \in \operatorname{simp} \tilde{\mathscr{B}} .} A\left(\mathscr{E}_{\sigma}\right) \times \operatorname{Lift}\left(\begin{array}{ccc} 
& & \tilde{\mathscr{B}} \\
& & \downarrow \\
B . & \stackrel{p}{\longrightarrow} & \mathscr{B} .
\end{array}\right) \rightarrow \operatorname{holim}_{\sigma \in \operatorname{simp} B .} A\left(E_{\sigma}\right)
$$

by the following rule: Given a simplicial map $q_{.}: B . \rightarrow \tilde{\mathscr{B}}$. lifting $p$, then $q$. induces a functor $q_{*}: \operatorname{simp} B . \rightarrow \operatorname{simp} \tilde{\mathscr{B}}$. Restriction along $q_{*}$ defines a map from the homotopy limit space appearing in the left-hand side to the one in the right-hand side.

The commutative diagram

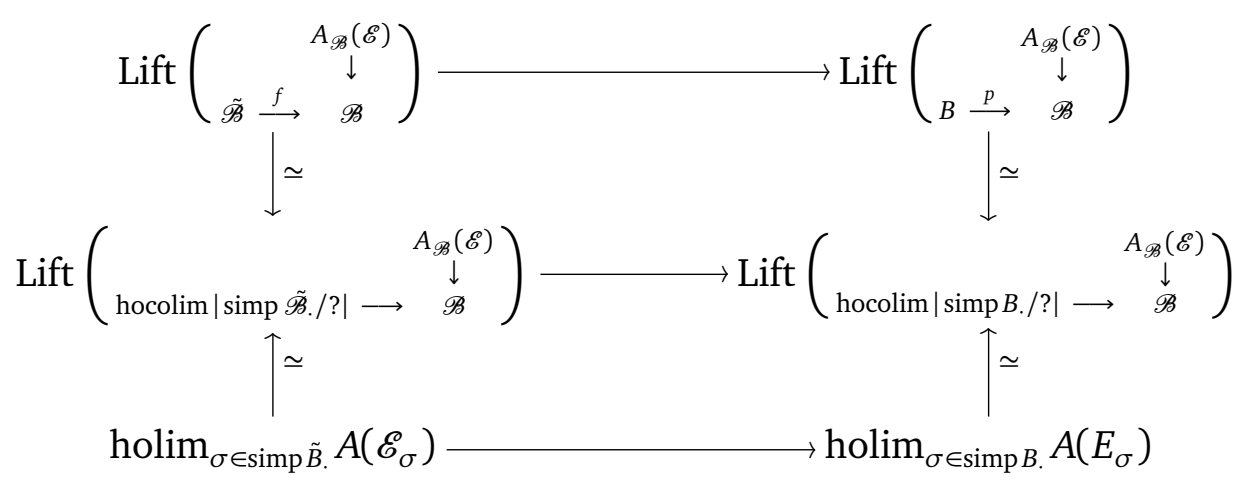

would then show that under the passage to section spaces, this map corresponds to the restriction operation in (2).

The above rule works fine if we are given a single simplicial map $q .: B . \rightarrow$ $\mathscr{B}$., i.e. a 0 -simplex in the corresponding simplicial set of lifts. However already a 1 -simplex in the space of lifts is given by a simplicial map $q .: B . \times \Delta^{1} \rightarrow$ $\tilde{\mathscr{B}}$, and there is no canonical way to obtain a path in the right-hand side joining the images of the two faces of $q$. Instead we get an element in holim $_{\sigma \in \operatorname{simp}\left(B, \times \Delta^{1}\right)} A\left(E_{\sigma}\right)$.

The remedy is to define a simplicial space $X$. by

$$
X_{n}:=\operatorname{holim}_{\sigma \in \operatorname{simp}\left(B . \times \Delta^{n}\right)} A\left(E_{\sigma}\right) .
$$

The simplicial structure is given by restriction along the correspdonding functors between categories of simplices. Since the inclusion

$$
\text { c. } \operatorname{holim}_{\sigma \in \operatorname{simp} B .} A\left(E_{\sigma}\right)=c . X_{0} \rightarrow X \text {. }
$$


of the constant simplicial object is an objectwise weak homotopy equivalence, we obtain a weak homotopy equivalence after thick realization in the sense of Segal [Seg74] (i.e. geometric realization of the underlying $\Delta$-space).

The thick realization of $X$. has a loop space structure compatible with the one on $X_{0}$. Indeed, let $X_{n} \rightarrow \Omega Y_{n}$ be a simplicial homotopy equivalence. It induces

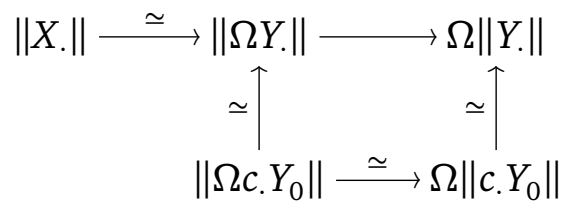

Now our rule from above defines a simplicial map

$$
\text { c. } \operatorname{holim}_{\sigma \in \operatorname{simp} \tilde{\mathscr{B}} .} A\left(\mathscr{E}_{\sigma}\right) \times \operatorname{Lift}\left(\begin{array}{ccc} 
& & \tilde{\mathscr{B}} \\
& & \downarrow \\
B . & \stackrel{p}{\longrightarrow} & \mathscr{B} .
\end{array}\right) \rightarrow X .
$$

whose thick realization is compatible with the loop space structures. This will be our model for the map (4). (Notice that there is a homeomorphism

$$
\|c . A \times B .\| \cong A \times\|B .\|
$$

for any simplicial space $B$. and any space $A$.)

The suitably adapted version of diagram (5) shows that under passage to section spaces, this map still corresponds to restriction.

Now the same arguments work for the functor $A^{\%}$ instead of $A$, and since everything is natural, we obtain a map on homotopy fibers.

\subsection{Composition rule and homeomorphism invariance}

Here and in the following, we suppose that all bundles $p: E \rightarrow B$ are such that the parametrized Whitehead torsion map is defined, i.e. we suppose that $p \in \operatorname{Bun}_{n}(B ; F)$ with a base space $B$ which is homotopy equivalent to a locally finite simplicial complex.

The essential properties of the classical Whitehead torsion generalize to the parametrized version. The behaviour under composition is a formal consequence of the naturality properties of the excisive characteristic: 
Proposition 3.8 (Composition rule). Let $\varphi: p \rightarrow p^{\prime}$ is a fiber homotopy equivalence. Then, the following diagram commutes up to homotopy:

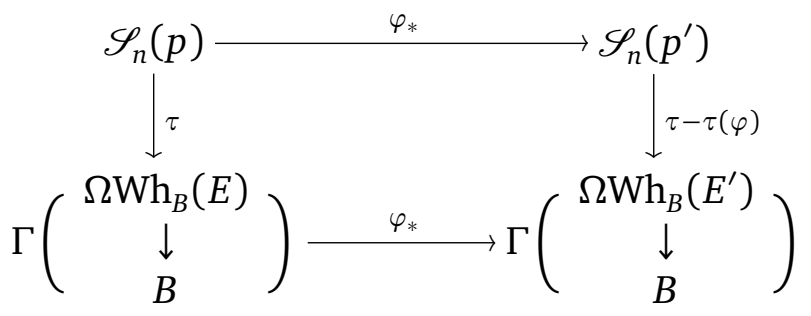

Proof. To be precise, the left hand vertical map is the map $\tau\left(\cdot, \mathrm{id}_{p}\right)$ whereas the right hand vertical map is the map

$$
\tau\left(\cdot, \mathrm{id}_{p^{\prime}}\right)-\tau\left(\varphi, \mathrm{id}_{p^{\prime}}\right)=\left(\chi^{\%}-\chi^{\%}\left(\operatorname{id}_{p^{\prime}}\right)\right)-\left(\chi^{\%}(\varphi)-\chi^{\%}\left(\operatorname{id}_{p^{\prime}}\right)\right)=\chi^{\%}-\chi^{\%}(\varphi)
$$

up to homotopy. Now the commutativity of the diagram follows from the naturality of the excisive characteristic.

The homeomorphism invariance of the parametrized torsion is built in the definitions:

Remark 3.9. If $\varphi: E^{\prime} \rightarrow E$ is a fiber homeomorphism of bundles, then $\tau(\varphi)=$ 0 . In fact, the fiber homeomorphism provides $\varphi \in \mathscr{S}_{n}(p)$ with a canonical nullhomotopy.

\subsection{The torsion on the stable structure space}

So far we defined for each space $\mathscr{S}_{n}(p), n \in \mathbb{N}$, a parametrized torsion map $\tau=: \tau_{n}$. This map is well-defined only up to homotopy since it involved a choice of a representative for $\chi^{\%}\left(\tilde{\mathscr{P}}_{n}\right)=: \chi_{n}^{\%}$ and $\chi(\mathscr{P})=: \chi$ for the universal "bundles" $\tilde{\mathscr{P}}_{n}$ (over $\tilde{\mathscr{B}}_{n}:=\operatorname{Bun}_{n}(* ; F)$ ) and $\mathscr{P}$ (over $\mathscr{B}:=\mathrm{Fib}(* ; F)$ ).

Now consider the stabilization map

$$
S: \mathscr{S}_{n}(p) \rightarrow \mathscr{S}_{n+1}(p)
$$

which on $k$-simplices is defined by the rule

$$
\left(E^{\prime} \rightarrow E\right) \mapsto\left(E^{\prime} \times I \rightarrow E^{\prime} \rightarrow E\right)
$$

where $E^{\prime}$ is a bundle of $n$-manifolds over $B \times \Delta^{k}$. 
(Strictly speaking, the unit interval is here a fixed subset of our universe $\mathscr{U}$. We then can make use of the fixed bijection $\mathscr{U} \times \mathscr{U} \rightarrow \mathscr{U}$ to consider $E^{\prime} \times I$ as a subset of $B \times \Delta^{k} \times \mathscr{U}$.)

Under the weak homotopy equivalence

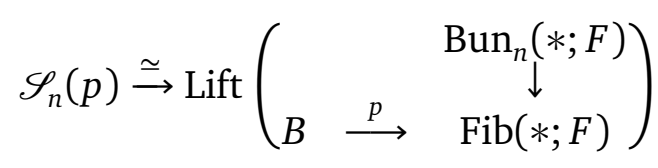

the stabilization looks as follows: Given $p^{\prime}: B \rightarrow \operatorname{Bun}_{n}(* ; F)$ and a homotopy $H$ from projection of $p^{\prime}$ to $p$, we have

$$
S\left(p^{\prime}, H\right)=\left(S\left(p^{\prime}\right), H * K\right)
$$

where $S: \operatorname{Bun}_{n}(* ; F) \rightarrow \operatorname{Bun}_{n+1}(* ; F)$ is the obvious stabilization map and $K$ is the canonical homotopy coming from the fiber homotopy equivalence between the bundle $p^{\prime}$ and its stabilization $S\left(p^{\prime}\right)$.

The stabilization map is a special case of a map

$$
\times X: \mathscr{S}_{n}(p) \rightarrow \mathscr{S}_{n+k}(p)
$$

defined for any contractible compact $k$-manifold $X$, given as a subset of $\mathscr{U}$.

The goal of this section is to extend the individual maps $\tau_{n}$ to a stabilized torsion

$$
\tau: \mathscr{S}(p):=\underset{n}{\operatorname{hocolim}} \mathscr{S}_{n}(p) \rightarrow \Gamma\left(\begin{array}{c}
\Omega \mathrm{Wh}_{B}(E) \\
\downarrow \\
B
\end{array}\right) .
$$

The main ingredient for this will be a lax naturality of the excisive characteristic:

Theorem 3.10. (i) Let $X$ be a contractible compact manifold, $p: E \rightarrow B$ a bundle of compact topological manifolds, and consider the bundle $p^{\prime}=$ $p \circ \pi: E^{\prime}=E \times X \rightarrow E \rightarrow B$. The canonical path

$$
\pi_{*} \chi\left(p^{\prime}\right) \rightsquigarrow \chi(p)
$$

between the Dwyer-Weiss-Williams homotopy invariant characteristics, induced by $\pi$, lifts along the fiberwise assembly map $\alpha$, up to homotopy relative endpoints, to a path

$$
\pi_{*} \chi^{\%}\left(p^{\prime}\right) \rightsquigarrow \chi^{\%}(p)
$$

between the Dwyer-Weiss-Williams excisive characteristics. 
(ii) If $Y$ is another contractible compact manifold, let $p^{\prime \prime}: E \times X \times Y \rightarrow B$ be the corresponding bundle. If $\pi^{\prime}: E \times X \times Y \rightarrow E \times X$ is the projection, then the two possible ways of lifting the path

$$
\pi_{*}^{\prime} \pi_{*} \chi\left(p^{\prime \prime}\right) \rightsquigarrow \chi(p)
$$

are homotopic relative endpoints.

(See the proof below for the formal definition of the excisive characteristic and of the map $\pi_{*}$.)

Now, to be able to define the stabilized torsion, we have to relate the choices we made for the different representatives $\chi_{n}^{\%}$. To do that, denote by

$$
\tilde{\mathscr{B}}:=\underset{n}{\operatorname{hocolim}} \tilde{\mathscr{B}}_{n}
$$

the homotopy colimit over the stabilization maps. There is an induced mapping $f: \tilde{\mathscr{B}} \rightarrow \mathscr{B}$ and we have

$$
\mathscr{S}(p) \simeq \operatorname{Lift}\left(\begin{array}{lll} 
& & \tilde{\mathscr{B}} \\
& & \downarrow \\
B & \stackrel{p}{\longrightarrow} & \mathscr{B}
\end{array}\right) .
$$

Hence, to obtain a parametrized Whitehead torsion in the same way as in section 3.2, we need to produce a homotopy class of lifts in the diagram

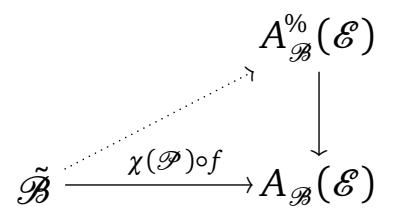

Now there is a commutative diagram

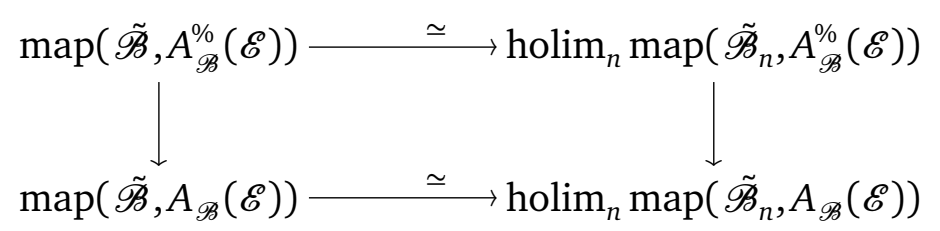

where the horizontal maps are homotopy equivalences. Notice that in the right-hand side, for each $n$ the vertical homotopy fiber over $\chi\left(\mathscr{P}_{n}\right) \circ f$ is a space of lifts which can be interpreted completely in terms of homotopy limits, by Lemma 3.3 . 
Remark 3.11. If

$$
\ldots \stackrel{f_{3}}{\rightarrow} X_{2} \stackrel{f_{2}}{\rightarrow} X_{1} \stackrel{f_{1}}{\rightarrow} X_{0}
$$

is a diagram indexed by the naturals, then a map $g: A \rightarrow \operatorname{holim}_{i} X_{i}$ into the homotopy limit is given by a sequence of maps $g_{i}: A \rightarrow X_{i}$ together with a sequence of homotopies between $g_{i}$ and $f_{i+1} \circ g_{i+1}$.

Using this remark, we may now apply of Theorem 3.10, together with the naturality properties of the Dwyer-Weiss-Williams characteristics in Lemma 3.1 to obtain an element in the upper right-hand side of diagram (7). Its projection in the lower right-hand side will agree with the image of $\chi(\mathscr{P}) \circ f$ under the lower horizontal map. Hence we obtain up to homotopy an element in the upper left-hand side projecting to $\chi(\mathscr{P}) \circ f$, i.e. a lift in the diagram (6).

\section{Excisive characteristics and cell-like projections}

This section deals with the proof of Theorem 3.10. We will prove this Theorem using the formal definition of the excisive characteristic, which we recall here.

For a simplicial set $B$. with geometric realization $B$, and a fiber bundle $p: E \rightarrow B$ of compact manifolds, let $t B$. be the simplicial set where an $n$ simplex is an $n$-simplex $\sigma$ of $B$., together with an equivalence relation $\theta$ on $E_{\sigma}:=|\sigma|^{*} E$, with quotient space $E_{\sigma}^{\theta}$, such that the projections induce a homeomorphism $E_{\sigma} \rightarrow \Delta^{n} \times E_{\sigma}^{\theta}$ over $|\sigma| \cong \Delta^{n}$. Then, the functor from simp $t B$. to spaces, sending $(\sigma, \theta)$ to $E_{\sigma}^{\theta}$, maps all objects to compact ENRs and all morphisms to homeomorphisms (which are cell-like maps). Thus we obtain a characteristic for the functor

$$
\sigma \mapsto \mathscr{R}^{\%}(X)
$$

By geometric realization and the map $\left|\mathscr{R}^{\%}(X)\right| \rightarrow K\left(\mathscr{R}^{\%}(X)\right) \rightarrow A^{\%}(X)$ we therefore get an excisive characteristic

$$
\chi_{e}(p) \in \underset{(\sigma, \theta)}{\operatorname{holim}} A^{\%}\left(E_{\sigma}^{\theta}\right)
$$

The projection $\operatorname{holim}_{\sigma} A^{\%}\left(E_{\sigma}\right) \rightarrow \operatorname{holim}_{(\sigma, \theta)} A^{\%}\left(E_{\sigma}^{\theta}\right)$ is a homotopy equivalence. Hence up to homotopy, the excisive characteristic defines a section in a suitable fibration:

$$
\chi_{e}(p) \in \Gamma\left(\begin{array}{c}
A_{B}^{\%}(E) \\
\downarrow \\
B
\end{array}\right) .
$$


Moreover the images of $\chi(p)$ and $\chi_{e}(p)$ in holim ${ }_{(\sigma, \theta)} A\left(E_{\sigma}^{\theta}\right)$ are connected by a canonical path. We obtain therefore an element in

$$
\begin{aligned}
\chi^{\%}(p) \in \operatorname{holim}\left(\operatorname{holim} A\left(E_{\sigma}\right) \stackrel{\simeq}{\rightarrow} \underset{(\sigma, \theta)}{\operatorname{holim}} A\left(E_{\sigma}^{\theta}\right) \leftarrow \underset{(\sigma, \theta)}{\operatorname{holim}} A^{\%}\left(E_{\sigma}^{\theta}\right)\right) \\
\simeq \operatorname{holim}\left(\operatorname{holim}_{\sigma} A^{\%}\left(E_{\sigma}\right) \rightarrow \underset{\sigma}{\operatorname{holim}} A\left(E_{\sigma}\right)\right)
\end{aligned}
$$

which projects to $\chi(p)$.

Let us denote by $t B$. and $t^{\prime} B$. the simplicial sets obtained by applying the above construction to the bundles $p$ resp. $p^{\prime}$. We now introduce a third simplicial set $t t^{\prime} B$., where an $n$-simplex consists of a triple $\left(\sigma, \theta, \theta^{\prime}\right)$ such that $(\sigma, \theta)$ and $\left(\sigma, \theta^{\prime}\right)$ are $n$-simplices of $t B$. and $t^{\prime} B$., respectively, and the respective homeomorphisms fit into a commutative diagram of the form

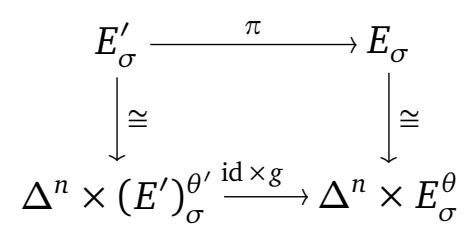

There are forgetful simplicial maps $t t^{\prime} B$. $\rightarrow t B$. and $t t^{\prime} B$. $\rightarrow t^{\prime} B$.; the first map has a section: it maps $(\sigma, \theta)$ to $\left(\sigma, \theta, \theta^{\prime}\right)$, with $\theta^{\prime}$ the equivalence relation on $E_{\sigma}^{\prime} \cong E_{\sigma} \times X$ such that $(e, x) \theta^{\prime}\left(e^{\prime}, x^{\prime}\right)$ if and only if $x=x^{\prime}$ and $e \theta e^{\prime}$. These maps induce pull-back maps

$$
\begin{gathered}
\operatorname{holim}_{(\sigma, \theta)} A^{\%}\left(E_{\sigma}^{\theta}\right) \stackrel{f}{\stackrel{f}{\rightleftarrows}} \underset{\left(\sigma, \theta, \theta^{\prime}\right)}{\operatorname{holim}} A^{\%}\left(E_{\sigma}^{\theta}\right) \\
\underset{\left(\sigma, \theta^{\prime}\right)}{\operatorname{holim} A^{\%}}\left(\left(E^{\prime}\right)_{\sigma}^{\theta^{\prime}}\right) \stackrel{g}{\rightarrow} \underset{\left(\sigma, \theta, \theta^{\prime}\right)}{\operatorname{holim}} A^{\%}\left(\left(E^{\prime}\right)_{\sigma}^{\theta^{\prime}}\right)
\end{gathered}
$$

Denote by $\bar{\chi}_{e}(p) \in \operatorname{holim}_{\left(\sigma, \theta, \theta^{\prime}\right)} A^{\%}\left(E_{\sigma}^{\theta}\right)$ the corresponding characteristic for the functor $\left(\sigma, \theta, \theta^{\prime}\right) \mapsto A^{\%}\left(E_{\sigma}^{\theta}\right)$, and define $\bar{\chi}_{e}\left(p^{\prime}\right)$ similarly.

The category $\operatorname{simp} t t^{\prime} B$. is defined such that $\pi$ induces a cell-like natural transformation $\bar{\pi}:\left(E^{\prime}\right)_{\sigma}^{\theta^{\prime}} \rightarrow E_{\sigma}^{\theta}$.

Claim. There is a canonical path from $\bar{\pi}_{*} \bar{\chi}^{\%}\left(p^{\prime}\right)$ to $\bar{\chi}^{\%}(p)$.

Proof of claim. The transformation $\bar{\pi}$ can be regarded as a functor $F$ from $\operatorname{simp} t t^{\prime} B . \times 1$ to spaces which takes all morphisms to cell-like maps, and therefore comes with a characteristic

$$
\bar{\chi}_{e} \in \operatorname{holimp}_{\operatorname{sim} t^{\prime} B . \times 1} F \cong \operatorname{holim}_{x \in 1} \operatorname{holim}_{\operatorname{simp} t t^{\prime} B . \times x} F .
$$

Spelled out, $\bar{\chi}_{e}$ consists of 
(i) the characteristic $\bar{\chi}_{e}\left(p^{\prime}\right) \in \operatorname{holim}_{\left(\sigma, \theta, \theta^{\prime}\right)} A^{\%}\left(\left(E^{\prime}\right)_{\sigma}^{\theta^{\prime}}\right)$,

(ii) the characteristic $\bar{\chi}_{e}(p) \in \operatorname{holim}_{\left(\sigma, \theta, \theta^{\prime}\right)} A^{\%}\left(E_{\sigma}^{\theta}\right)$, and

(iii) a path from $\bar{\pi}_{*} \bar{\chi}_{e}\left(p^{\prime}\right)$ to $\bar{\chi}_{e}(p)$.

This proves the claim.

Therefore, since $g\left(\chi_{e}\left(p^{\prime}\right)\right)=\bar{\chi}_{e}\left(p^{\prime}\right)$, and $f\left(\chi_{e}(p)\right)=\bar{\chi}_{e}(p)$, we can apply $s$ to the canonical path from the claim, to obtain a canonical path

$$
s \circ \bar{\pi}_{*} \circ g\left(\chi_{e}\left(p^{\prime}\right)\right) \rightsquigarrow s \circ f\left(\chi_{e}(p)\right)=\chi_{e}(p) .
$$

Defining $\pi_{*}$ to be the composite $s \circ \pi_{*} \circ g$, we have found the path we were looking for.

To see that the path obtained in this way is a lifting of the canonical path on the level of homotopy invariant characteristics, recall that our path was constructed as an excisive characteristic $\bar{\chi}_{e}$ for a functor on $\operatorname{simp} t t^{\prime} B . \times 1$. The canonical path on the level of homotopy invariant characteristics comes similarly from a characteristic $\chi$ of a functor with domain $\operatorname{simp} B . \times 1$. The argument that gives a canonical path from $\alpha\left(\chi_{e}(p)\right)$ to $\chi(p)$ now applies to give a canonical path from $\alpha\left(\bar{\chi}_{e}\right)$ to $\bar{\chi}$.

Now notice that under the projections

$$
\underset{\sigma}{\operatorname{holim}} A\left(E_{\sigma}\right) \rightarrow \underset{(\sigma, \theta)}{\operatorname{holim}} A\left(E_{\sigma}^{\theta}\right) \quad \text { and } \operatorname{holim}_{\sigma} A\left(E_{\sigma}^{\prime}\right) \rightarrow \underset{\left(\sigma, \theta^{\prime}\right)}{\operatorname{holim}} A\left(\left(E^{\prime}\right)_{\sigma}^{\theta}\right)
$$

the map $s \circ \pi_{*} \circ g$ corresponds to the map induced by the projection $E_{\sigma}^{\prime} \rightarrow E_{\sigma}$.

This completes the proof of part (i) of the Theorem. Part (ii) can be proven similarly by constructing a category $\operatorname{simp} t t^{\prime} t^{\prime \prime} B$. and considering the excisive characteristic

$$
\bar{\chi}^{\%} \in \operatorname{holimp}_{\operatorname{sim} t t^{\prime \prime} t_{. \times 2}} F .
$$

\subsection{A product formula}

In this section we formulate a particular case of a product formula, which is adapted to our needs. It is also a consequence of Theorem 3.10 .

Recall the map

$$
\times X: \mathscr{S}_{n}(p) \rightarrow \mathscr{S}_{n+k}(p)
$$


defined in subsection 3.4 for a contractible compact manifold $X^{k}$. The for two such manifolds $X^{k}, Y^{l}$, the diagram

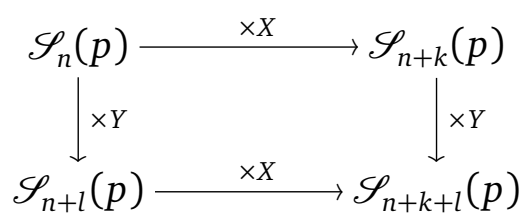

commutes up to a canonical homotopy, which comes from the bijection

$$
(\mathscr{U} \times \mathscr{U}) \times \mathscr{U} \cong \mathscr{U} \times(\mathscr{U} \times \mathscr{U}) .
$$

Applying this to $Y:=I$, we therefore obtain a stabilized version

$$
\times X: \mathscr{S}(p) \rightarrow \mathscr{S}(p) .
$$

Theorem 3.12. The following diagram commutes up to homotopy

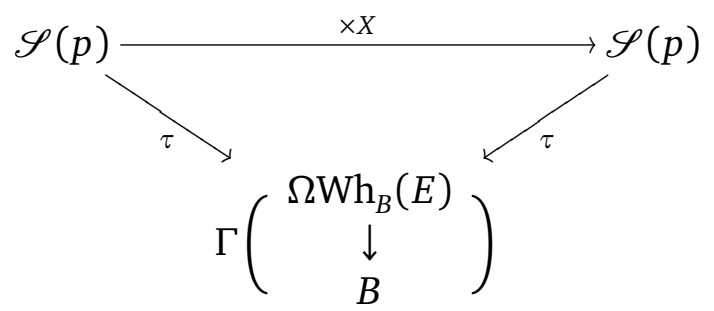

Proof. The maps $(\times X): \tilde{\mathscr{B}}_{n} \rightarrow \tilde{\mathscr{B}}_{n+k}$ assemble to a map

$$
(\times X): \tilde{\mathscr{B}} \rightarrow \tilde{\mathscr{B}} \text {. }
$$

We have to show that the lift

$$
\underset{\mathscr{\mathscr { B }}}{\stackrel{A_{\mathscr{B}}^{\%}(\mathscr{E})}{\longrightarrow}{ }^{(\mathscr{P}) \circ f} \underset{\mathscr{B}}{\longrightarrow}(\mathscr{E})}
$$

used in the definition of the stable parametrized torsion does not change under restriction with the map $(\times X)$. Again we use the translation (7) together with Lemma 3.3. Hence we need to give:

(i) For each $n$, a path $\gamma_{n}$ between $\chi^{\%}\left(\tilde{\mathscr{P}}_{n} \times X\right)$ and $\chi^{\%}\left(\tilde{\mathscr{P}}_{n}\right)$. This is given by part (i) of Theorem 3.10.

(ii) For each $n$, a homotopy between the path $\gamma_{n}$ and the restriction of the path $\gamma_{n+1}$ under the stabilization map. This is given by part (ii) of Theorem 3.10 . 


\subsection{Additivity}

One of the major properties of classical Whitehead torsion is additivity; we now proceed to formulate a parametrized version. Let $B$ be homotopy equivalent to locally finite simplicial complex, and let us form a category where an object is an object of $\mathbf{F i b}(B ; F)$ for some homotopy finitely dominated space $F$, and a morphism $p \rightarrow p^{\prime}$ is a fiberwise map from $p$ to $p^{\prime}$. With this language, let $\square$ denote the following commutative diagram in this category

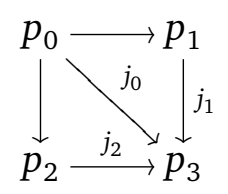

where we assume that all maps on the level of total spaces are cofibrations and that the total space $E\left(p_{3}\right)$ is the push-out of the total spaces $E\left(p_{1}\right)$ and $E\left(p_{2}\right)$ over $E\left(p_{0}\right)$.

By an $n$-dimensional structure on $\square$, we mean an extension of this diagram to a commutative cube

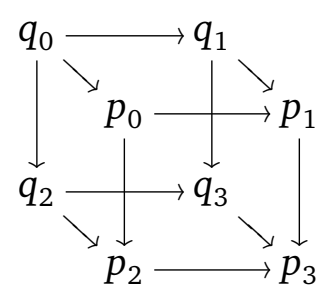

such that $q_{i}$ are objects of the categories $\operatorname{Bun}_{n}\left(B ; F_{i}\right)$ for $i=1,2,3$ respectively, $q_{0}$ is an object of $\operatorname{Bun}_{n-1}\left(B ; F_{0}\right)$, all the maps $q_{i} \rightarrow p_{i}$ are fiber homotopy equivalences, and the $q$-square is a codimension 1 splitting of $q_{3}$. By this we mean that all bundles $q_{i}$ are locally flat subbundles of $q_{3}$, of codimension 0 for $i=1,2$ and of codimension 1 of $i=0$, and that the total space of $q_{0}$ is the intersection of the total spaces of $q_{1}$ and $q_{2}$. The $n$-dimensional structures on $\square$ form the zeroth term of a simplicial set $\mathscr{S}_{n}(\square)$., with $k$-simplices such diagrams parametrized over $\Delta^{k}$. Let $\mathscr{S}_{n}(\square)$ be its geometric realization. It comes with forgetful maps $\alpha_{i}: \mathscr{S}_{n}(\square) \rightarrow \mathscr{S}_{n}\left(p_{i}\right)$ for $i=1,2,3$, and $\alpha_{0}: \mathscr{S}_{n}(\square) \rightarrow \mathscr{S}_{n-1}\left(p_{0}\right)$. 
Theorem 3.13 (Additivity). The following diagram commutes up to homotopy:

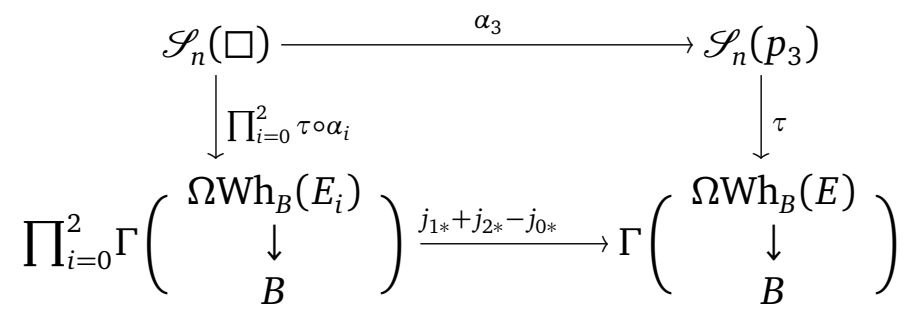

Explanation. As pointed out in section 3.2, the loop space structure used to define the addition operation in the lower line of the diagram is defined between spaces which are weakly homotopy equivalent to the given ones. So the precise statement of Theorem 3.13 is

$$
j_{1 *} \circ \tau \circ \alpha_{1}+j_{2 *} \circ \tau \circ \alpha_{2}-j_{0 *} \circ \tau \circ \alpha_{0} \simeq \tau \circ \alpha_{3} .
$$

We will prove the proposition more generally for the excisive characteristic $\chi^{\%}$ instead of the torsion $\tau$. The proof consists first by describing $\mathscr{S}_{n}(\square)$ as a suitable space of lifts, by considering universal bundles and fibrations with splittings. This will follow from the machinery presented in section 2 if we can establish certain formal properties of the "bundle theories" involved. Then we will make use of additivity results for the Dwyer-Weiss-Williams homotopy invariant and excisive characteristics, obtained by [Dor02] and [BD07], respectively.

The fibers of each of the fibrations in $\square$ fit into a diagram

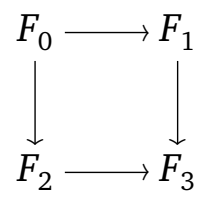

which we are going to abbreviate $\left(F_{i}\right)$. Now consider, for a space $B$, the category $\operatorname{Fib}\left(B ;\left(F_{i}\right)\right)$ of all diagrams (9) satisfying the conditions stated there, such that $p_{i}$ is an object of $\mathbf{F i b}\left(B ; F_{i}\right)$ for all $i$. Morphisms are commuting diagrams of fiber homotopy equivalences. Pulling back with $f: B^{\prime} \rightarrow B$ defines an object in $\operatorname{Fib}\left(B^{\prime} ;\left(F_{i}\right)\right)$. In fact, the cofibration condition still holds on the induced fibrations by Corollary A.10.

As a result, we obtain a functor

$$
\operatorname{Fib}\left(B ;\left(F_{i}\right)\right): \mathbf{c p C W}^{o p} \rightarrow \text { cat }
$$


and therefore a simplicial category. Finally we obtain a simplicial set

$$
\operatorname{Fib}\left(B ;\left(F_{i}\right)\right) .:=N_{0} \operatorname{Fib}\left(B ;\left(F_{i}\right)\right) .
$$

Similarly consider the category $\operatorname{Bun}_{n}\left(X ;\left(F_{i}\right)\right)$, which is the category of diagrams (9) of fibrations over $X$, such that $p_{0}$ is an object of $\operatorname{Bun}_{n-1}\left(B ; F_{0}\right), p_{i}$ are elements in $\operatorname{Bun}_{n}\left(B ; F_{i}\right)$ for $i=1,2,3$, and the square is a codimension 1 splitting of $p$. A morphism in this category is to be a commuting diagram of fiberwise homeomorphisms. This category gives rise to a simplicial set $\operatorname{Bun}_{n}\left(B ;\left(F_{i}\right)\right)$.

Lemma 3.14. The functors $\operatorname{Bun}_{n}\left(B ;\left(F_{i}\right)\right)$ and $\operatorname{Fib}\left(B ;\left(F_{i}\right)\right)$ satisfy the Amalgamation property, the Straightening property, and the Fill-in property if $B$ is metrizable and ULC.

Proof. The case of bundles being similar to the case in section 2, we focus on fibrations. For the Amalgamation property, we have to check that the cofibration condition still holds after glueing. This is verified in Proposition A.14. For the Straightening property, first use homotopy lifting to straighten each of the fibrations, and then use cofibration arguments to make the diagrams strictly commutative. Finally, the Fill-in property is proved in Proposition A.19.

Therefore, whenever $B$ is homotopy equivalent to a locally finite ordered simplicial complex, there is a weak homotopy equivalence

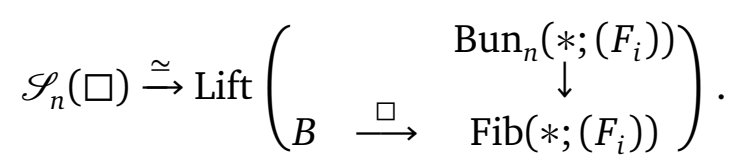

Denote, for by $\mathscr{Q}$ the universal "bundle" over $\operatorname{Fib}\left(* ;\left(F_{i}\right)\right)$, with induced "bundles" $\mathscr{Q}_{i}$ over $\operatorname{Fib}\left(* ; F_{i}\right)$ for $i=0, \ldots, 3$. The symbols $\tilde{\mathscr{Q}}$ and $\tilde{\mathscr{Q}}_{i}(i=0, \ldots, 3)$ will denote the universal "bundles" over $\operatorname{Bun}_{n}\left(* ;\left(F_{i}\right)\right)$ and their induced "bundles".

Theorem 3.15 ([Dor02, BD07]). (i) On the level of Dwyer-Weiss-Williams A-theory characteristics, there is a canonical path

$$
j_{1 *} \chi\left(\mathscr{Q}_{1}\right)+j_{2 *} \chi\left(\mathscr{Q}_{2}\right)-j_{0 *} \chi\left(\mathscr{Q}_{0}\right) \rightsquigarrow \chi\left(\mathscr{Q}_{3}\right) .
$$

(ii) The path from (i) lifts, up to homotopy relative endpoints, to a path

$$
j_{1 *} \chi^{\%}\left(\mathscr{Q}_{1}\right)+j_{2 *} \chi^{\%}\left(\mathscr{Q}_{2}\right)-j_{0 *} \chi^{\%}\left(\mathscr{Q}_{0}\right) \rightsquigarrow \chi^{\%}\left(\mathscr{Q}_{3}\right)
$$

between the Dwyer-Weiss-Williams excisive characteristics. 
Now Lemma 3.7 (with $\tilde{\mathscr{B}}$ replaced by $\tilde{\mathscr{B}}_{3}$ ) shows that this kind of additivity for the Dwyer-Weiss-Williams excisive characteristic produces the same kind of additivity for the parametrized excisive characteristic that we defined. This completes the proof of Theorem 3.13 .

Sketch of proof of Theorem 3.15 Let us first focus on a bundle over a point, i.e. a single manifold, $M$, with a codimension 1 splitting $M=M_{1} \cup_{M_{0}} M_{2}$. Let

$$
\bar{M}:=M_{1} \times 0 \cup_{M_{0} \times I} M_{2} \times I
$$

which comes with inclusions $i_{0}: M_{0} \times I \rightarrow \bar{M}, i_{1}: M_{1} \rightarrow \bar{M}, i_{2}: M_{2} \rightarrow \bar{M}$.

There are obvious cell-like projections $\bar{M} \rightarrow M$ and $M_{0} \times I \rightarrow M_{0}$, inducing path $\chi^{\%}(\bar{M}) \rightsquigarrow \chi^{\%}(M)$ and $\chi^{\%}\left(M_{0} \times I\right) \rightsquigarrow \chi^{\%}\left(M_{0}\right)$ by the lax naturality of the characteristic. Next, the cofibration sequences

$$
\begin{aligned}
M_{1} \coprod M_{2} \hookrightarrow \bar{M} \rightarrow \Sigma M_{0} \\
M_{1} \hookrightarrow M_{1} \coprod M_{2} \rightarrow M_{2} \\
M_{0} \hookrightarrow \text { cone } M_{0} \rightarrow \Sigma M_{0}
\end{aligned}
$$

produce, using the additivity theorem, the desired path

$$
i_{1 *} \chi^{\%}\left(M_{1}\right)+i_{2 *} \chi^{\%}\left(M_{2}\right)-i_{0 *} \chi^{\%}\left(M_{0} \times I\right) \rightsquigarrow \chi^{\%}(\bar{M}) .
$$

This completes the proof of the unparametrized statement. All the constructions are sufficiently natural to generalize to the $G$-equivariant case, if $G$ is a discrete group acting on $M$ in a way that respects the codimension 1 splitting.

Finally the theorem of Mather-Thurston-Segal-McDuff about the homology of classifying spaces of diffeomorphisms allows the passage from the discrete group of all homeomorphisms of $M$ that respect the codimension 1 splitting to the corresponding continuous group.

Remark. In [BD07], the statement is only formulated for closed manifolds. However the proof actually shows the more general case of compact manifolds.

This proof of Theorem 3.15 can easily be generalized to a stabilized version. It results that Theorem 3.13 has a stable version, too: 
Theorem 3.16. The following diagram commutes up to homotopy:

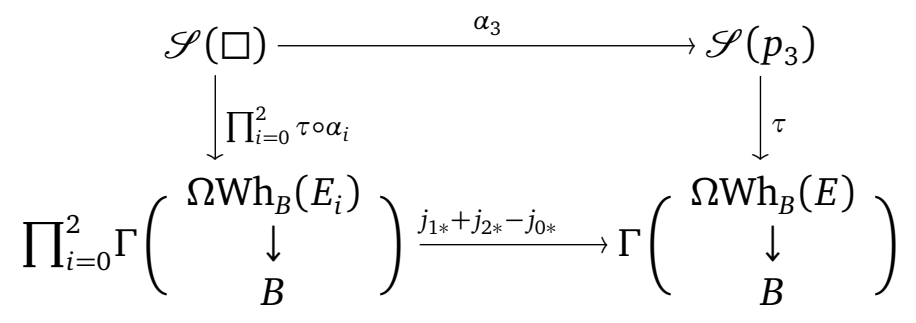

\subsection{Relation to $h$-cobordisms}

The relation between the structure space and higher algebraic $K$-theory via the torsion suggests that there is a link to parametrized $h$-cobordism theory. In fact, given a parametrized $h$-cobordism on a manifold $M$, the choice of a retraction of the cobordism onto $M$ can be understood as parametrized structure on $M$. So we may apply the torsion to obtain a map

$$
\mathscr{H}(M) \rightarrow \Omega \mathrm{Wh}(M)
$$

On the other hand, Waldhausen [Wal82] gave another description of a map relating the space of $h$-cobordisms on $M$ to higher algebraic $K$-theory. We prove in Theorem 3.18 that both maps agree. This builds on the reformulation of Waldhausen's map in the language of characteristics, performed in [DWW03, §§ 9 and 10]

It follows that for a fiber bundle $p: E \rightarrow B$ of compact topological manifolds, the section space

$$
\Gamma\left(\begin{array}{c}
\Omega \mathrm{Wh}_{B}(E) \\
\downarrow \\
B
\end{array}\right)
$$

can be identified with a stable space of fiberwise $h$-cobordisms on $p$. A precise statement using concordance stable ranges will be given in Corollary 3.19.

Let $p: E \rightarrow B$ be a fiber bundle of compact topological manifolds. Define $\mathscr{H}(p)_{0}$ to be the set of commutative diagrams

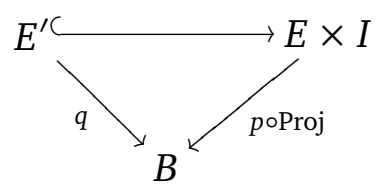


where $q$ is a bundle of compact topological manifolds over $B$ (as a subset of $B \times \mathscr{U}$ ) such that $E^{\prime}$ contains a neighborhood of $E \times\{0\}$, and the inclusion of the fibers of $p$ into the fibers of $q$ is a homotopy equivalence. We will think of $E^{\prime}$ as a fiberwise $h$-cobordism over $E$.

Definition 3.17. The space of $h$-cobordisms on $p$ is the geometric realization $\mathscr{H}(p)$ of the simplicial set with $k$-simplices $\mathscr{H}(p)_{k}:=\mathscr{H}\left(p \times \mathrm{id}_{\Delta^{k}}\right)_{0}$

Suppose that the fiberwise dimension of $p$ is $k$. The retraction of $E^{\prime}$ to $E \times 0$ is a homotopy equivalence, so there is a canonical map

$$
\mathscr{H}(p) \rightarrow \mathscr{S}_{k+1}(p) .
$$

Theorem 3.18. For a compact $k$-manifold $M$, the composite

$$
\mathscr{H}(M) \rightarrow \mathscr{S}_{k+1}(M) \stackrel{\tau}{\rightarrow} \Omega \mathrm{Wh}(M)
$$

is homotopic to Waldhausen's map.

Corollary 3.19. The composite

$$
\mathscr{H}(p) \rightarrow \mathscr{S}_{k+1}(p) \stackrel{\tau}{\rightarrow} \Gamma\left(\begin{array}{c}
\Omega \mathrm{Wh}_{B}(E) \\
\downarrow \\
B
\end{array}\right)
$$

is $(K-\operatorname{dim}(B)+1)$-connected, if $K$ is in the concordance stable range for the fibers of $p$.

Proof. If $B$ is a point, then the infinite stabilization $\mathscr{H}(M) \rightarrow \mathscr{H}_{\infty}(M)$ if $(K+$ 1)-connected by the definition of the concordance stable range, and $\mathscr{H}_{\infty}(M) \simeq$ $\Omega W h(M)$. In the case of a general $B$, use the fact that both the left-hand and the right-hand side are "co-excisive" in $B$, i.e. given a homotopy push-out $B_{3}=B_{1} \cup_{B_{0}} B_{2}$ of spaces over $B$, the diagram

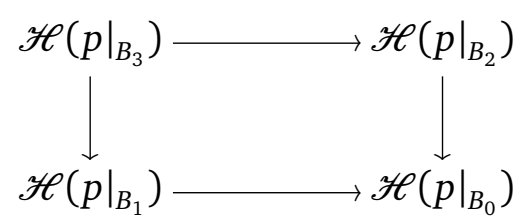

is a homotopy pull-back. It follows (see e.g. [DWW03, p. 62]) that the coassembly map

$$
\mathscr{H}(p) \rightarrow \operatorname{holim}_{\sigma \in \operatorname{simp} B} \mathscr{H}\left(\left.p\right|_{\sigma}\right)
$$


is a homotopy equivalence. Thus it is enough to prove the statement for

$$
\operatorname{holim}_{\sigma \in \operatorname{simp} B .} \mathscr{H}\left(p_{|\sigma|}\right) \rightarrow \operatorname{holim}_{\sigma \in \operatorname{simp} B} \Gamma\left(\begin{array}{c}
\Omega \mathrm{Wh}_{|\sigma|}\left(E_{\sigma}\right) \\
\downarrow \\
|\sigma|
\end{array}\right)
$$

Now it follows from the Bousfield-Kan spectral sequence that this map is $(N-\operatorname{dim} B)$-connected whenever all the maps for the individual $\sigma$ are $N$ connected.

Corollary 3.20. If $\operatorname{dim}(B)-1$ is less than the concordance stable range for the fibers, there is a one-to-one corresponcence between isomorphism classes of $h$ cobordisms on $p$ and elements of

$$
\pi_{0} \Gamma\left(\begin{array}{c}
\Omega \mathrm{Wh}_{B}(E) \\
\downarrow \\
B
\end{array}\right) .
$$

Proof of Theorem 3.18. Let us first describe the idea of the proof: Suppose we are given an $h$-cobordism $E$ over $M$, and denote by $i: M \rightarrow E$ the inclusion. In the description of [DWW03], Waldhausen's map basically considers $E$ as a retractive space over $M$, thus giving a point in $A(M)$, together with the refinement $W(E) \in A^{\%}(M)$ coming from the fact that $E$ is an ENR. The cofibration sequence of retractive spaces over $E$

$$
M \amalg E \hookrightarrow E \amalg E \rightarrow E \cup_{M} E
$$

together with Waldhausen's Additivity theorem show that

$$
i_{*} W(E)+i_{*} \chi^{\%}(M) \simeq \chi^{\%}(E) \in \operatorname{hofib}_{\chi(E)}\left(A^{\%}(E) \rightarrow A(E)\right) .
$$

Now apply the projection $\pi: M \rightarrow E$ (coming from the inclusion $E \subset M \times$ $I)$. Letting $\tau(i)$ denote the image of the $h$-cobordism, viewed as a manifold structure on $M$, under the Whitehead torsion, we obtain

$$
\tau(i)=\pi_{*} \chi^{\%}(E)-\chi^{\%}(M) \simeq W(E) .
$$

This argument works fine for a single $h$-cobordism. To obtain a parametrized version, one produces characteristics on certain functors, as in the definition of the $A$ - and $A^{\%}$-theory characteristics.

Firstly, consider the category simp $\mathscr{H}(M)$. of simplices in the simplicial set $\mathscr{H}(M)$.. An object of this category is mainly is a fiber bundle $E$ over $|\sigma|=\Delta^{n}$ 
which is an $h$-cobordism over $M \times \Delta^{n}$. We may consider $E$ as a retractive space over $M \times \Delta^{n}$ and use the functoriality with respect to the inclusion $M \times \Delta^{n} \subset E$ to obtain a retractive space over $E$. It is the characteristic object of a characteristic for the functor

$$
F: \operatorname{simp} \mathscr{H}(M) \rightarrow \text { cat, } \quad E \mapsto \mathscr{R}^{f d}(E)
$$

As in subsection 3.1, this characteristic yields an element

$$
W_{h} \in \operatorname{holim}_{E \in \operatorname{simp} \mathscr{H}(M) .} A(E)
$$

To define the refinement of this to a characteristic for $\mathscr{R}^{\%}\left(M \times \Delta^{n}\right)$, define a simplicial set $t \mathscr{H}(M)$ where an $n$-simplex is a bundle $E$ over $\Delta^{n}$ which is an $h$-cobordism over $M \times \Delta^{n}$ as above, together with an equivalence relation $\theta$ on $E$ such that

- the projections induce a homeomorphism $E \rightarrow \Delta^{n} \times E / \theta$, and

- in a neighborhood of $M \times \Delta^{n} \times 0$, the equivalence relation $\theta$ agrees with the one induced by the projection $M \times \Delta^{n} \times I \rightarrow \Delta^{n} \times I$.

This bigger simplicial set allows, similarly to the definition of the excisive characteristic, to define a characteristic for the functor

$$
\operatorname{simp} t \mathscr{H}(M) \rightarrow \text { cat, } \quad(E, \theta) \mapsto \mathscr{R}^{\%}(E / \theta)
$$

which we call "Waldhausen characteristic". By geometric realization, we obtain a characteristic

$$
W_{e} \in \operatorname{holim}_{(E, \theta) \in \operatorname{simp} t \mathscr{H}(M) .} A^{\%}\left(E_{\sigma}^{\theta}\right)
$$

which refines $W_{h}$ in the sense of subsection 3.4 .

Now there is a canonical projection $t \mathscr{H}(M) \rightarrow t \mathrm{Bun}_{k+1}(* ; M)$ (see the proof of Theorem 3.10 for notation). Recall that we used a characteristic on $\operatorname{simp} t \operatorname{Bun}_{k+1}(* ; M)$ to define the excisive characteristic, which we may therefore pull back to $\operatorname{simp} t \mathscr{H}(M)$ to compare it with the Waldhausen characteristic. We obtain, for each object of $\operatorname{simp} t \mathscr{H}(M)$, a cofibration sequence (10). These sequences fit together (see e.g. [BD07, Lemma 4.3]) to define a parametrized version of (11). 


\subsection{Comparison with the unparametrized case}

The aim of this section is to give an elementary argument that the parametrized Whitehead torsion reduces to the classical Whitehead torsion in the unparametrized setting. It is the linking part between classical simple homotopy theory and higher algebraic $K$-theory.

Of course, this is also implied by Theorem 3.18 from the last section, which however implicitly used all the machinery from Waldhausen's manifold models for algebraic $K$-theory, which actually are not needed in our argument.

This section expands on the sketch that appeared in [FLS09, section 11].

Proposition 3.21. Let $M$ be a compact topological n-manifold. Then, the map

$$
\tau: \pi_{0} \mathscr{S}_{n}(M) \rightarrow \pi_{0} \Omega \mathrm{Wh}(M) \cong \mathrm{Wh}(\pi M)
$$

sends $f: N \rightarrow M$ to the Whitehead torsion of $f$.

The first thing to notice is that $\pi_{0} \mathscr{S}_{n}(M)$ is the structure set of compact $n$-manifolds in the usual sense, i.e. the set of homotopy equivalences $N \rightarrow M$ from compact $n$-manifolds, where such a homotopy equivalence is identified with $N^{\prime} \rightarrow M$ if and only if there is a homeomorphism $N \rightarrow N^{\prime}$ making the obvious diagram homotopy commutative. Notice also that $\pi_{0}$ of the codomain of the excisive characteristic map is

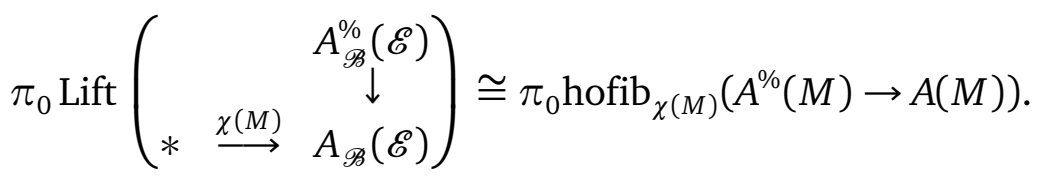

Consider a homotopy equivalence $\phi: N \rightarrow M$ of compact $n$-manifolds. The proof of the proposition will consist in identifying the path component of $\tau(\phi)$ with a concrete element of $\pi_{0}$ hofib $_{\chi(M)}\left(A^{\%}(M) \rightarrow A(M)\right)$, given using Waldhausen's description of the $A$-theoretic assembly map as in [Wal85, chapter $3]$. This will allow to make use of Waldhausen's model of the homotopy fiber of the assembly map. The path components of this model provide a geometric description of the Whitehead group, which is isomorphic to the standard algebraic one.

We will make use of Waldhausen's notation from [Wal85] without further remarks. The models we will use are

$$
\begin{aligned}
A^{\%}(X) & =\Omega\left|s S . \mathscr{R}_{f}\left(X^{\Delta}\right)\right|, \\
A(X) & =\Omega\left|w S . \mathscr{R}_{f}\left(X^{\Delta}\right)\right|, \\
\Omega W h(X) & =\left|s \mathscr{C}_{f}^{h}(X)\right|
\end{aligned}
$$


The assembly map $A^{\%}(X) \rightarrow A(X)$ is induced by the inclusion of categories $s \mathscr{R}_{f}(X) \rightarrow w \mathscr{R}_{f}(X)$.

Remarks. (i) This model for $A(X)$ has $\pi_{0} A(X)=\mathbb{Z}$, whereas the one used by Dwyer-Weiss-Williams has $K_{0}(\mathbb{Z}[\pi(X)])$ as path components. But since here all spaces are homotopy finite, the path component of their characteristic, which is the Euler characteristic, lies in $\mathbb{Z}$, so the homotopy fiber is the same.

(ii) Waldhausen's models presented here are functors from simplicial sets and not from spaces. So we assume all manifolds to be triangulated. Thus, strictly speaking, we have to replace the manifold $M$ by a triangulated closed disc bundle $A$ over $M$, which is always possible by [KS77, Essay 3].

Consider the composites

$$
\begin{aligned}
\left|s \mathscr{R}_{f}(X)\right| & \rightarrow \Omega\left|s S . \mathscr{R}_{f}(X)\right| \rightarrow \Omega\left|s S . \mathscr{R}_{f}\left(X^{\Delta}\right)\right|=A^{\%}(X) \\
\left|w \mathscr{R}_{f}(X)\right| & \rightarrow \Omega\left|w S . \mathscr{R}_{f}(X)\right| \rightarrow \Omega\left|w S . \mathscr{R}_{f}\left(X^{\Delta^{\prime}}\right)\right|=A(X)
\end{aligned}
$$

The first maps in both composites are the maps reminiscent of the group completion, whereas the second maps come from the inclusion $A_{0} \rightarrow A$. which exist for any simplicial space $A$. For any manifold $M$, the object $M \amalg m$ of $\mathscr{R}_{f}(M)$ (with obvious section and retraction) defines an element $\chi^{\%}(M) \in A^{\%}(M)$ and an element $\chi(\phi) \in A(M)$. Since $\alpha \chi^{\%}(M)=\chi(M)$, we even obtain an element

$$
\chi^{\%}(\phi) \in \pi_{0} \operatorname{hofib}_{\chi(M)}\left(A^{\%}(M) \rightarrow A(M)\right) .
$$

We are going to call this element Waldhausen's excisive characteristic. (This characteristic is not excisive in the sense of [DWW03], but this difference is not important here. See the introduction of [Wei02] for a more detailed discussion.)

Notice that the homotopy invariant characteristic $\chi$ defined here is the same as the one of Dwyer-Weiss-Williams, modulo the homotopy equivalence $\Omega\left|w S . \mathscr{R}_{f}(X)\right| \rightarrow \Omega\left|w S . \mathscr{R}_{f}\left(X^{\Delta^{*}}\right)\right|$. Not surprisingly, the excisive characteristics agree as well. This is a consequence of the following three observations:

(i) Both definitions of the excisive characteristic agree certainly if $M$ is contractible, since in this case the assembly map is a homotopy equivalence.

(ii) Moreover both definitions are additive in the sense of Proposition 3.13 (in the unparametrized setting on $\pi_{0}$ level). The proof in the Waldhausen setting is basically identical to the proof for the homotopy invariant characteristic. 
Now, given a homotopy equivalence $\phi: N \rightarrow M$ between compact manifolds, the path component of the element

$$
\tau(\phi)=\chi^{\%}(M)-\phi_{*} \chi^{\%}(N) \in \pi_{0} \operatorname{hofib}_{*}\left(A^{\%}(M) \rightarrow A(M)\right)
$$

is represented by the object cone $(\phi)$, considered as a retractive space over $M$ in the obvious way. This is seen using the cofibration sequence in $\mathscr{R}_{f}(M)$

$$
M \amalg M r \operatorname{cone}(\phi) \rightarrow \Sigma(M \amalg N) .
$$

Now we only need to observe that the space $\operatorname{cone}(\phi)$, as a space relative to $M$, also defines an object of the category $\mathscr{C}_{f}^{h}(M)$ and therefore an element in $\Omega \mathrm{Wh}(M)=\left|s \mathscr{C}_{f}^{h}(M)\right|$. It represents $\tau(\phi)$ under the isomorphism $\pi_{0} \Omega \mathrm{Wh}(M) \cong \pi_{0} \operatorname{hofib}_{*}\left(A^{\%}(M) \rightarrow A(M)\right)$.

To complete the proof, observe that $\pi_{0} \Omega \mathrm{Wh}(M)$ is just the geometric definition of the Whitehead group, so under the isomorphism $\pi_{0} \Omega \mathrm{Wh}(M) \cong$ $\mathrm{Wh}(\pi M)$ (see e.g. [Coh73]), the element $\tau(\phi)$ corresponds to the Whitehead torsion of $\phi$. 


\section{The geometric assembly map}

This section is devoted to the definition and study of the "geometric assembly map" on structure spaces. The first result will be Theorem 4.2 which asserts that the geometric assembly map on the level of stable structure spaces has an "algebraic" counterpart on the level of algebraic $K$-theory. Using Hoehn's theorem and some microbundle theory, we deduce in section 4.3 that the square appearing in Theorem 4.2 is actually a weak homotopy pull-back. This result will be used for the treatment of fibering questions.

The last two sections are devoted to show that these two results also hold on the level of $Q$-manifolds. This will allow to treat fibering questions on $Q$-manifolds using the same techniques.

\subsection{Definition of the geometric assembly map}

The total space of a bundle of $n$-dimensional compact manifolds over a $k$ dimensional compact manifold is itself an $(n+k)$-dimensional compact manifold. Therefore, given a fibration $p: E \rightarrow B$, there is a product map

$$
\mathscr{S}_{k}(B) \times \mathscr{S}_{n}(p) \rightarrow \mathscr{S}_{n+k}(E)
$$

which on $m$-simplices is defined as follows: If $x \in \mathscr{S}_{k}(B)_{m}$ and $y \in \mathscr{S}_{n}(p)_{m}$ are given by
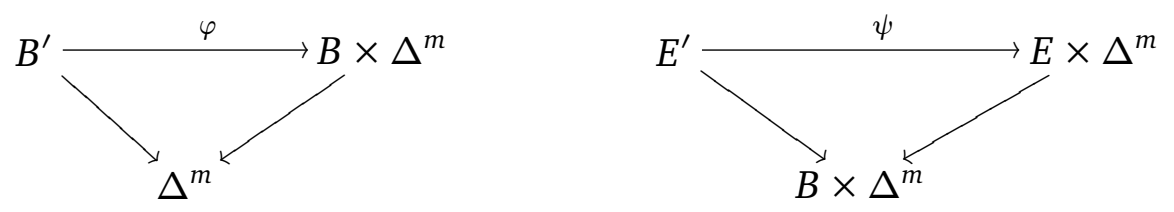

then the image of $(x, y)$ is given by $\varphi^{*} E^{\prime}$, considered as a bundle over $\Delta^{m}$, together with its canonical map to $E \times \Delta^{m}$.

Again, strictly speaking, we have to care about the universes: the bundle given by the image of $(x, y)$ must be a subset of $\Delta^{m} \times \mathscr{U}$, whereas it is canonically a subset of $\Delta^{n} \times \mathscr{U} \times \mathscr{U}$. Make therefore use of the fixed bijection $\mathscr{U} \times \mathscr{U} \rightarrow \mathscr{U}$. 
In particular, if $B$ is itself a $k$-dimensional compact manifold, the identity map on $B$ defines a point in $S_{k}(B)$; hence we can evaluate the product map to obtain a map

$$
\alpha: \mathscr{S}_{n}(p) \rightarrow \mathscr{S}_{n+k}(E) .
$$

Again, strictly speaking, we must embed $B$ as a subset of $\mathscr{U}$. However, the homotopy class of $\alpha$ does not depend on this choice.

Geometrically $\alpha$ takes all the structures on the fibers of $p$ and assembles them into one big structure. Therefore we are going to call $\alpha$ the geometric assembly map. It should not be confused with the abstract notion of assembly map as defined by [WW95].

Remark 4.1. If $B$ is a point, then the map $\alpha: \mathscr{S}_{n}(E) \rightarrow \mathscr{S}_{n}(E)$ is canonically homotopic to the identity map.

In fact, $\alpha(x)$ and $x$ are canonically homeomorphic. This homeomorphism provides a homotopy.

\subsection{Geometric assembly and torsion I}

The aim of this section is to give an "algebraic" description of $\alpha$ in the following sense: We defined parametrized Whitehead torsion maps, going from geometrically defined structure spaces to "algebraically" (or more precisely homotopy theoretically) defined section spaces involving algebraic $K$-theory of spaces. It is natural to ask whether in the diagram

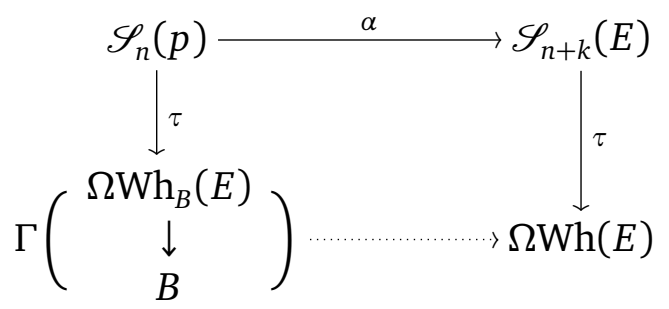

a dotted arrow exists which makes the diagram commutative.

Therefore suppose for simplicity that $B$ is path-connected, and define another map $\alpha$ as the following composition (after choosing a point $b \in B$ ):

$$
\alpha: \Gamma\left(\begin{array}{c}
\Omega \mathrm{Wh}_{B}(E) \\
\downarrow \\
B
\end{array}\right) \stackrel{\text { Restriction }}{\longrightarrow} \Omega \mathrm{Wh}\left(F_{b}\right) \stackrel{\chi_{e}(B) \cdot j_{*}}{\longrightarrow} \Omega \mathrm{Wh}(E) .
$$


Here $j_{*}$ is induced by the inclusion of the fiber $F_{b}$ into $E$ and $\chi_{e}$ denotes the Euler characteristic. Notice that a different choice of base point $b$ leads to the same map $\alpha$, up to homotopy.

Theorem 4.2. If $p$ is a bundle of compact topological manifolds over a compact connected topological manifold, the following diagram commutes up to homotopy:

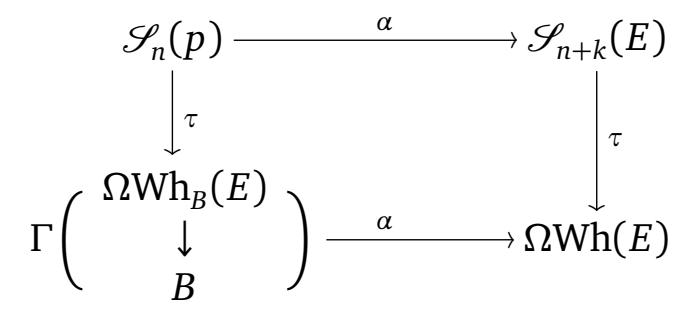

Before giving a proof, let us first formulate a stabilized version of this theorem. It is the stable version which will be important for our treatment of fibering questions. Recall the definition

$$
\mathscr{S}(p)=\underset{n}{\operatorname{hocolim}} \mathscr{S}_{n}(p)
$$

from section 3.4. Recall also that the torsion induces a map

$$
\tau: \mathscr{S}(p) \rightarrow \Gamma\left(\begin{array}{c}
\Omega \mathrm{Wh}_{B}(E) \\
\downarrow \\
B
\end{array}\right)
$$

whenever $p: E \rightarrow B$ is a bundle of compact topological manifolds.

On the other hand, if $B$ is a compact topological manifold, the geometric assembly map induces a map

$$
\alpha: \mathscr{S}(p) \rightarrow \mathscr{S}(E)
$$

since $\alpha$ commutes with stabilization up to a canonical homotopy.

Theorem 4.3. If $p$ is a bundle of compact topological manifolds over a compact connected topological manifold, the following diagram commutes up to homotopy:

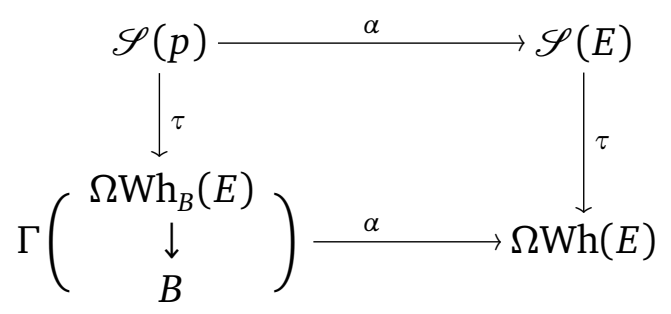


The proof of the two theorems will occupy the rest of this section. The main part of the proof consists of the following two statements.

(i) If $X$ is a compact contractible manifold, then the claim holds for $p$ if and only if the claim holds for the bundle $p \times \mathrm{id}_{X}$.

(ii) If $B=B_{1} \cup_{B_{0}} B_{2}$ is a codimension 1 splitting of the base manifold, then the claim holds for $p$ whenever it holds for the restrictions of $p$ onto $B_{0}, B_{1}$, and $B_{2}$.

Proof of Theorem 4.2, using statements (i) and (ii). We first consider the case where the base space is a contractible manifold. In this case $p$ is fiber homeomorphic to a product bundle $p^{\prime} \times \mathrm{id}_{B}: F \times B \rightarrow * \times B$. By the homeomorphism invariance of the torsion, we may thus suppose that $p$ is a product bundle. Thus, using the statement (i), we are left to show that the claim holds when the base space of the bundle is the one-point space. But then both maps $\alpha$ (on the level of structure spaces and on the $A$-theoretic side) are just (homotopic to) the identity.

The next step is to give a proof for $B=S^{n}$. The case $n=0$ is trivial using remark 4.1 (with suitably adjusted statement, as $S^{0}$ is not connected). Otherwise, $S^{n}=D^{n} \cup_{S^{n-1}} D^{n}$ has a codimension 1 splitting along the equator, and inductively the claim holds by statement (ii) and what we already proved.

Suppose now that $p$ is an arbitrary fibration. By statement (i), we can prove the claim for $p$ by proving the claim for the fibration $p \times \mathrm{id}_{D^{n}}$. Therefore, we can assume that the dimension of $B$ is greater than 6 , such that $B$ has a handlebody decomposition, by [KS77, Essay III, thm. 2.1].

Now we proceed by induction on the number $k$ of handles of the handlebody decomposition. We already dealt with the case $k=1$, since then $B=D^{n}$ is contractible. Suppose now that $B$ is obtained from $B^{\prime}$ by attaching a handle, such that $B=B^{\prime} \cup_{S^{m} \times D^{n-m-1}} D^{m+1} \times D^{n-m-1}$, and suppose that the claim is established for the fibration restricted to $B^{\prime}$. Then, by (ii) and what we already proved, the claim holds for $B$ as well.

Proof of statement (i). Denote by $p^{\prime}: E^{\prime} \rightarrow B^{\prime}$ the fibration $p \times \mathrm{id}_{X}$ and let $f: B^{\prime} \rightarrow B$ be the projection. Suppose that $\operatorname{dim} B=k$ and $\operatorname{dim} X=l$. The proof consists in considering the following diagram (in the homotopy category): 


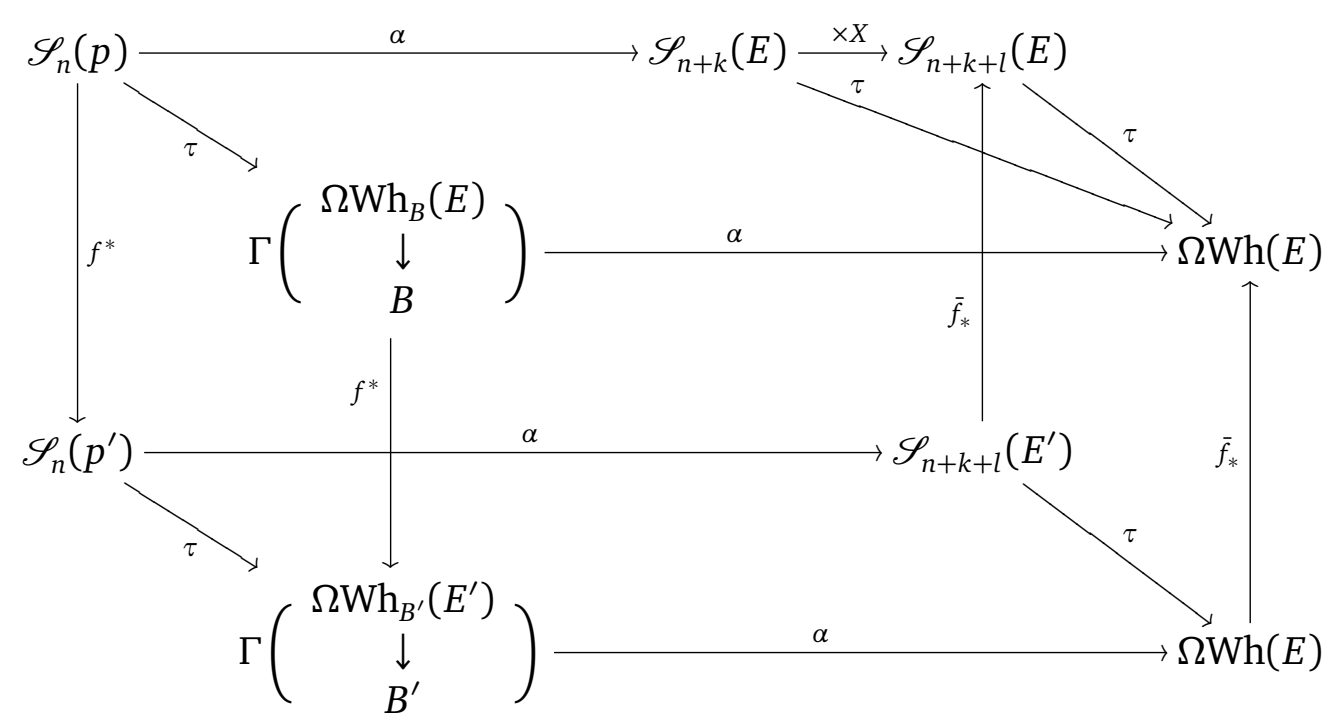

To show that the square on the top (resp. bottom) of the diagram commutes, it is enough to show that all the other squares and the triangle commute.

The left-hand square commutes by naturality. The front square commutes by the definition of $\alpha$. It is elementary to see that the back square also commutes.

The two remaining cases are the non-trivial ones: The right-hand square is commutative by the composition rule, since $\bar{f}$ is cell-like and therefore has torsion zero. The triangle commutes by our product formula, Theorem 3.12 .

Proof of statement (ii). Denote by $j_{i}: B_{i} \rightarrow B$ for $i=0,1,2$ the inclusion. Notice that the codimension 1 splitting of $B$ induces a codimension 1 splitting of $E$ as follows:

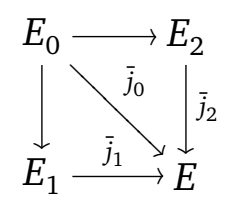

since $p$ is a bundle. Denote by $\square$ this diagram, thought of as a diagram of bundles over the one-point space. Recall the notation $\mathscr{S}_{n}(\square)$ from the additivity theorem of the parametrized torsion, and denote by $\gamma_{i}: \mathscr{S}_{n}(\square) \rightarrow \mathscr{S}_{n}\left(E_{i}\right)$ $(i=1,2), \gamma_{0}: \mathscr{S}_{n}(\square) \rightarrow \mathscr{S}_{n-1}\left(E_{0}\right)$ and $\gamma: \mathscr{S}_{n}(\square) \rightarrow \mathscr{S}_{n}(E)$ the forgetful maps.

The claim follows by considering the following diagram in the homotopy category: 


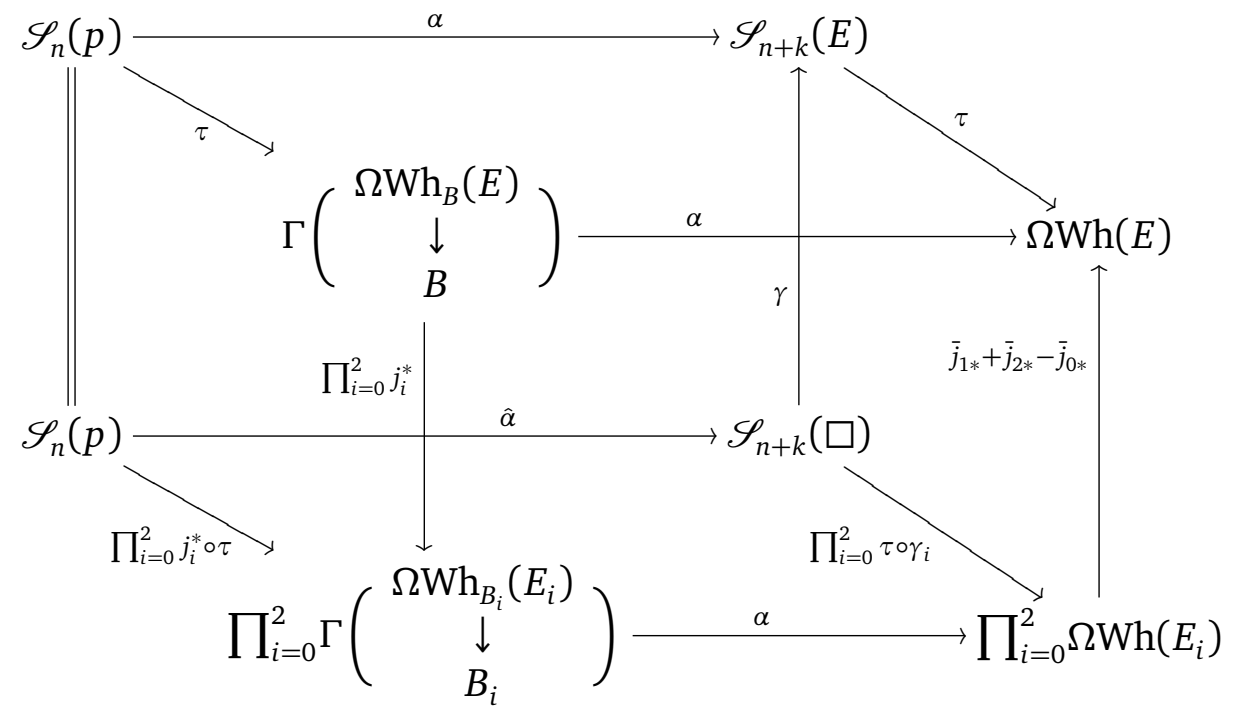

Here $\hat{\alpha}$ is to denote the obvious map which forgets the base space but remembers the codimension 1 splitting.

To show that the top square commutes, it is enough to check that all the other squares are commutative.

The commutativity of the left-hand square is purely formal, and the bottom square commutes by naturality of the torsion. The back square is evidently commutative. The commutativity of the front square follows from the definition of $\alpha$ and the additivity of the Euler characteristic,

$$
\chi_{e}(B)=\chi_{e}\left(B_{1}\right)+\chi_{e}\left(B_{2}\right)-\chi_{e}\left(B_{0}\right) .
$$

Finally, the Additivity theorem 3.13 guarantees that the right-hand square is commutative.

The proof of the "stable" Theorem 4.3 is identical to the proof of the unstable version 4.2 - one only needs to quote the stable versions of additivity and product rule instead of the unstable ones.

\subsection{Geometric assembly and torsion II}

One may wonder how much information gets lost under the parametrized torsion map. We already saw that $\tau$ stays invariant under stabilization (i.e. crossing with the unit interval). In this section we will see that it also stays invariant under change of tangential structure, and that this is all the ambiguity introduced by applying the torsion. This follows from the description 
of the structure space (Theorem 4.6) as given by Stacy Hoehn's PhD thesis [Hoe09], building on the parametrized A-theory characteristic and the stable parametrized $h$-cobordism theorem by Jahren-Rognes-Waldhausen [WJR08].

Applying some microbundle theory [Mil64], we obtain the following.

Theorem 4.4. The square

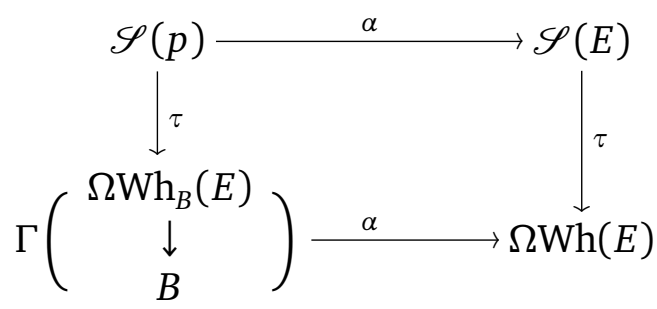

is a weak homotopy pull-back.

We start by recalling the tangent (micro-)bundle of a topological manifold $E$, which is represented by the diagram

$$
E \stackrel{\Delta}{\rightarrow} E \times E \stackrel{\text { Proj }_{1}}{\rightarrow} E,
$$

$\Delta$ denoting the diagonal inclusion and Proj $_{1}$ the projection onto the first factor. If $E$ is the total space of a fiber bundle of topological manifolds over $B$, then the fiberwise tangent bundle is the microbundle over $E$ defined by

$$
E \stackrel{\Delta}{\rightarrow} E \times{ }_{B} E \stackrel{\text { Proj }_{1}}{\longrightarrow} E .
$$

We would like to define a classifying space for microbundles. Therefore denote by $\mathbf{M B}_{n}(B)$ the category of all $n$-dimensional microbundles over $B$, considered as a subset of $B \times \mathscr{U}$, where the morphisms are isomorphismgerms between those in the sense of Milnor [Mil64, §6]. Pull-back gives rise to a functor

$$
\text { cpCW }^{o p} \rightarrow \text { cat, } \quad X \mapsto \mathrm{MB}_{n}(B \times X) .
$$

Lemma 4.5. This functor has the Amalgamation, Straightening, and Fill-in properties as defined in section 2

Hence, a microbundle over a locally finite ordered simplicial complex $B$ is, up to homotopy, the same thing as a map from $B$ to some classifying space. By results of Kister [Kis64] and Mazur [Maz64], every microbundle contains a pointed euclidean bundle as an open sub-bundle; this bundle is unique up to isomorphism. It follows that the classifying space for $n$-dimensional microbundles is homotopy equivalent to $B \mathrm{TOP}(n)$. 
Proof of Lemma. We begin with the Amalgamation property. Therefore let

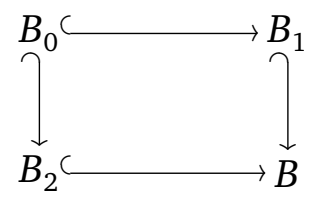

be a push-out of compact CW complexes, and let $E_{i}, i=0,1,2$, be microbundles over $B_{i}$ whose restrictions to $B_{0}$ agree. We have to show that the push-out of the $E_{i}$ defines a microbundle over $B$. Therefore we check local triviality which is clear for all $b \in B$ unless $b \in B_{0}$. So let $b \in B_{0}$.

Since any open neighborhood of the zero-section of a microbundle defines still a microbundle, we may suppose by Kister and Mazur that both $E_{0}$ and $E_{2}$ are in fact pointed euclidean bundles. Let $U_{1}$ be a neighborhood of $b$ in $B_{1}$ and $E_{1}^{0}$ be a neighborhood of $b$ in $E_{1}$ such that $E_{1}^{0} \cong U_{1} \times \mathbb{R}^{n}$ is in fact a trivial microbundle over $U$. In particular, the restriction $E_{0}^{0}$ of $E_{1}^{0}$ over $U_{0}:=U_{1} \cap B_{0}$ is also trivial.

Now, since the inclusion of $B_{0}$ into $B_{2}$ is a cofibration, $U_{0}$ extends to a neighborhood $U_{2}$ of $b$ which retracts to $U_{0}$ by a map $r: U_{2} \rightarrow U_{0}$ such that the following holds: there is an isomorphism of euclidean bundles

$$
\left.\left.E_{2}\right|_{U_{2}} \cong r^{*} E_{0}\right|_{U_{0}}
$$

which is the identity on $\left.E_{0}\right|_{U_{0}}$.

Consider the open neighborhood $E_{2}^{0}$ of $b$ inside $\left.E_{2}\right|_{U_{2}}$ which corresponds to $r^{*} E_{0}^{0}$ under this isomorphism. Then we have $E_{2}^{0} \cong r^{*} E_{0} \cong U_{2} \times \mathbb{R}^{n}$, and the isomorphisms fit together to define an isomorphism $E_{1}^{0} \cup E_{2}^{0} \rightarrow\left(U_{1} \cup U_{2}\right) \times \mathbb{R}^{n}$. This proves amalgamation.

The straightening property is proved in [Mil64, §6]. To obtain a fill-in, take mapping cylinders whenever we are given actual isomorphisms rather than just isomorphism-germs. In the general case observe that if $E^{0} \subset E$ is an open neighborhood of the zero-section in a microbundle over $B$, then the open subspace

$$
E \times[0,1) \cup E^{0} \times\{1\} \subset E \times[0,1]
$$

is a microbundle over $B \times[0,1]$ which is a fill-in between $E$ and $E^{0}$.

Hoehn indicates [Hoe09, section 1.2] how to define a map

$$
T_{\text {fib }}: \mathscr{S}(p) \rightarrow \operatorname{map}(E, B \text { TOP })
$$


which, informally, sends a diagram

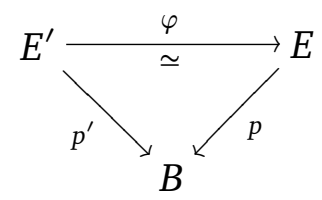

to $\left(\varphi^{-1}\right)^{*} T_{\text {fib }} p^{\prime}$, the (stabilized) fiberwise tangent bundle of $p^{\prime}$, pulled back to $E$ by some homotopy inverse of $\varphi$.

Formally, one first defines a larger structure space $\overline{\mathscr{S}}_{n}(p)$ where a 0-simplex is a diagram (2) together with a choice of fiber homotopy inverse $\psi$ of $\varphi$. The forgetful map $\overline{\mathscr{S}}_{n}(p) \rightarrow \mathscr{S}_{n}(p)$ is a homotopy equivalence; on the other hand the explicit choice of homotopy equivalence in $\overline{\mathscr{S}}_{n}(p)$ allows to define a map to the space of $n$-dimensional microbundles. Stabilizing yields a map to $\operatorname{map}(E, B T O P)$.

If $B$ is a point, we denote the map $T_{\text {fib }}$ simply by $T$. The fiberwise tangent bundle is of interest for us due to the following theorem [Hoe09, Theorem 3.3.2].

Theorem 4.6. If $p$ is a bundle of compact topological manifolds over a compact topological manifold, then the map

$$
\mathscr{S}(p) \stackrel{T_{\mathrm{fib}} \times \tau}{\longrightarrow} \operatorname{map}(E, B \mathrm{TOP}) \times \Gamma\left(\begin{array}{c}
\Omega \mathrm{Wh}_{B}(E) \\
\downarrow \\
B
\end{array}\right)
$$

is a weak homotopy equivalence.

Remark 4.7. Hoehn states the theorem with the assumption that $B$ is a finite CW complex. Using the naturality and homotopy invariance in $B$ of domain and target, one sees that the statement holds more generally for all base spaces that are homotopy equivalent to finite CW complexes. Recall that this is the way we defined the parametrized torsion for this class of base spaces.

Remark 4.8. Technically speaking, our definition of the tangent microbundle does not allow the manifolds to have boundaries. This problem can be dealt with by adding to all manifolds a collar at the boundary. Existence and uniqueness [KS77, Essay I, App. A] of collars shows that the corresponding structure spaces are homotopy equivalent. On the other hand, the collar allows to extend the tangent bundle of the interior of $M$ over the boundary. 
In the following theorem we make use of the $H$-group structure on the space $\operatorname{map}(E, B \mathrm{TOP})$ to obtain a homotopy equivalence

$$
+p^{*} \text { TB: } \operatorname{map}(E, B \text { TOP }) \rightarrow \operatorname{map}(E, B T O P)
$$

given by adding the pulled-back tangent bundle $p^{*} T B$.

Theorem 4.9. The diagram

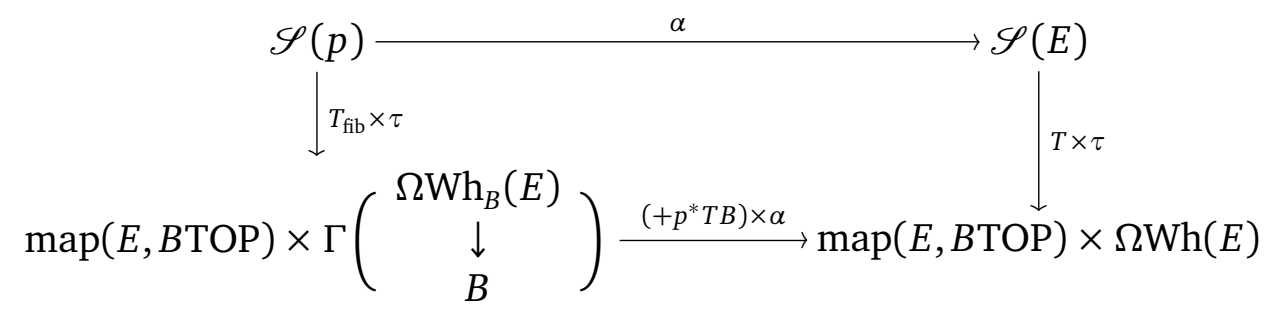

commutes up to homotopy.

Theorem 4.4 now clearly follows by combining the last two theorems, recalling that the map $\left(+p^{*} T B\right)$ is a homotopy equivalence.

To prove Theorem 4.9, one has to prove two separate statements: one involving only the maps $T_{\text {fib }}$ and $T$, and one involving only the maps $\tau$. The statement for $\tau$ has been proven in Theorem 4.3 .

Let us first proof the non-parametrized version of the statement involving the tangent bundles.

Lemma 4.10. Let $p: E \rightarrow B$ be a bundle of compact topological manifolds over a compact topological manifold $B$. Let $q: U \rightarrow B$ be a pointed $\mathbb{R}^{n}$-bundle representing the tangent microbundle $T B$ (as an open subbundle of the projection $B \times B \rightarrow B$ onto the first coordinate), with zero-section $s$.

Then any choice of fiber homotopy $\mathrm{id}_{U} \simeq s \circ q$ over $B$ defines a microbundle $X$ over $E \times I$ which restricts to $T E$ over $E \times 0$ and to $T_{\text {fib }} E \oplus p^{*} T B$ over $E \times 1$.

Thus, in particular, we have

$$
T E \cong T_{\mathrm{fib}} E \oplus p^{*} T B
$$

Proof. Denote by $p^{\prime}: V:=p^{*} U \rightarrow E$ the pointed $\mathbb{R}^{n}$-bundle pulled back from $U$ using the bundle map $p$. The space $V$ is open in $E \times B$, and the fiber homotopy $\mathrm{id}_{U} \simeq s \circ q$ induces a fiber homotopy

$$
H: V \times\left[\frac{1}{2}, 1\right] \rightarrow E \times B
$$


between the inclusion from $V$ into $E \times B$ and the composite $V \stackrel{p^{\prime}}{\rightarrow} E \stackrel{\Delta}{\hookrightarrow} E \times B$. Now consider the bundle

$$
\text { id } \times p: E \times E \rightarrow E \times B \text {. }
$$

Pulling it back using $H$, we obtain a bundle over $V \times\left[\frac{1}{2}, 1\right]$ which restricts to $E \times\left. E\right|_{V}$ over $V \times \frac{1}{2}$; over $V \times 1$, it restricts to $\left(p^{\prime}\right)^{*}\left(E \times_{B} E\right)$.

We may consider the bundle over $V \times\left[\frac{1}{2}, 1\right]$ as a microbundle over $E \times$ $\left[\frac{1}{2}, 1\right]$. As such, its restriction over $V \times 1$ is precisely the direct sum $T_{\text {fib }} E \oplus$ $p^{*} T B$, and its restriction over $V \times \frac{1}{2}$ is an open subbundle of $T E$ (which therefore is germ-isomorphic to $T E$ ). Using the fill-in property of microbundles, we get a microbundle over $V \times\left[0, \frac{1}{2}\right]$ which connects the open subbundle of $T E$ with TE.

Proof of Theorem 4.9. As observed above, it is enough to show that $T \circ \alpha$ is homotopic to $T_{\text {fib }}+p^{*} T B$. Let us first consider the unstable case. To obtain a homotopy, it is enough to construct a map

$$
\overline{\mathscr{S}}_{n}(p) \rightarrow \operatorname{map}(E \times I, B \mathrm{TOP})
$$

which sends a structure $x$ to a bundle that "interpolates" between $T_{\text {fib }}(x)+$ $p^{*} T B$ and $T(\alpha x)$. This is achieved simplicially using the construction in the proof of Lemma 4.10 .

For the stable case, observe that stabilization of structures induces stabilization of the corresponding bundles. So the compatibility is automatic.

Remark 4.11 (The smooth case). There is little doubt that the DIFF analogue of Theorem 4.4 is also true. Here are the main issues which arise if one tries to generalize the Theorem in this direction:

As we have seen, two main ingredients of the proof are the parametrized additivity and product rules. While it is easy to define a DIFF parametrized torsion using the approach we have taken, it seems difficult to prove the additivity and product rules this way, due to the fact the "unit transformation" $Q\left(X_{+}\right) \rightarrow A^{\%}(X)$ is defined only up to contractible choice. A better approach to the DIFF case seems to redefine the Dwyer-Weiss-Williams characteristics using Waldhausen's manifold models for A-theory [Wal82] in the spirit of [BDW09].

A further essential ingredient to the proof of Theorem 4.4 is Hoehn's Theorem 4.6. Here the problem is similar: the manifold model approach seems to be necessary for the DIFF case. 
Notice however that the mere commutativity of the square in Theorem 4.4 on the $\pi_{0}$-level does not depend on the parametrized additivity and product rule (but only on the non-parametrized one, which is classical), nor on Hoehn's theorem. It follows that if an element $f: M \rightarrow E$ in $\mathscr{S}(E)$ disassembles up to homotopy, the same will be true for its Whitehead torsion $\tau(f)$. In our appliations to fibering questions, this implies that the vanishing of the DIFF analogues of our obstructions is at least a necessary condition to fibering - so differences between the DIFF and the TOP case may be seen already at this stage.

\subsection{Geometric assembly on bundles of $Q$-manifolds}

Denote, as usual, by $Q$ the Hilbert cube, which is a countable product of unit intervals. A $Q$-manifold is a separable metric space which is locally homeomorphic to open subsets of $Q$. The theory of $Q$-manifolds provides the following results:

- If $X$ is a locally compact ANR, then $X \times Q$ is a $Q$-manifold (Edwards, see [Cha76, Theorem 44.1]).

- Every compact $Q$-manifold is of the form $X \times Q$, where $X$ is a compact polyhedron (Chapman, see [Cha76, Theorem 36.2]).

- Any cell-like map [Lac69] between Q-manifolds is a near-homeomorphism, i.e. is arbitrarily close to a homeomorphism (Chapman, see [Cha76, Corollary 43.2]). In particular, if there is a cell-like map $M \rightarrow N$ between $Q$-manifolds, then $M \cong N$. For example, $M \times Q \cong M$, the socalled "stability" of $Q$-manifolds.

- If $M$ and $N$ are compact $Q$-manifolds, then the space $\operatorname{Homeo}(M, N)$ of homeomorphisms from $M$ to $N$ (with the compact-open topology) is an ANR, in particular locally contractible (Ferry [Fer77]).

Let $p: E \rightarrow B$ be a fibration over a paracompact base space. By a $Q$ manifold structure on $p$ we mean a commutative diagram

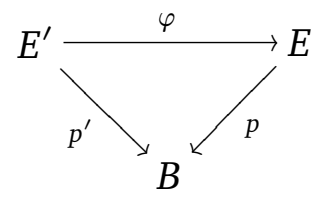

where $p^{\prime}$ is a bundle of compact $Q$-manifolds, and $\varphi$ is a homotopy equivalence. As with finite-dimensional structures, we assume $E^{\prime}$ to be a subset of 
$B \times \mathscr{U}$, with the inclusion map being a map over $B$. This gives rise to $\mathscr{S}^{Q}(p)$, the space of $Q$-manifold structures, which is (just as the space of compact $n$ dimensional manifolds) the geometric realization of a suitable simplicial set, with zero-simplices now the set of $Q$-manifold structures. If $B$ is a point, we write $\mathscr{S}^{Q}(E)$ for $\mathscr{S}^{Q}(p)$. Notice that the simplicial set $\mathscr{S}^{Q}(p)$. is Kan.

Lemma 4.12. If $p: E \rightarrow B$ is a bundle of $Q$-manifolds over a locally compact $A N R$, then $E$ is also a Q-manifold.

Proof. Let $e \in E$, and let $U \subset B$ be an open neighborhood of $p(e)$ over which the bundle is trivial. An open subset of an ANR is itself an ANR [Han51, Lemma 3.1], so $\left.E\right|_{U} \cong U \times F$ is a locally compact ANR. Hence, $\left.E\right|_{U} \times Q$ is a $Q$-manifold. On the other hand, $\left.E\right|_{U} \times Q \cong U \times F \times Q \cong U \times\left. F \cong E\right|_{U}$ by the stability of $Q$-manifolds.

Therefore, in analogy with the finite-dimensional case, we have a geometrically defined assembly map

$$
\alpha: \mathscr{S}^{\mathrm{Q}}(p) \rightarrow \mathscr{S}^{\mathrm{Q}}(E)
$$

whenever $B$ is a compact ANR. Strictly speaking, as in the finite-dimensional case, this map is defined if $B$ is a subset of $\mathscr{U}$, which we shall tacitly assume from now on.

We would like to compare the space of $Q$-manifold structures with the space of $n$-dimensional compact manifold structures. To do that, consider again the stabilization map

$$
\times I: \mathscr{S}_{n}(p) \rightarrow \mathscr{S}_{n+1}(p) .
$$

On the other hand, there is a similarly defined map

$$
\times Q: \mathscr{S}_{n}(p) \rightarrow \mathscr{S}^{Q}(p)
$$

by taking fiberwise the product with $Q$.

Lemma 4.13. (i) If $B$ is a compact n-manifold then the following diagram commutes up to homotopy:

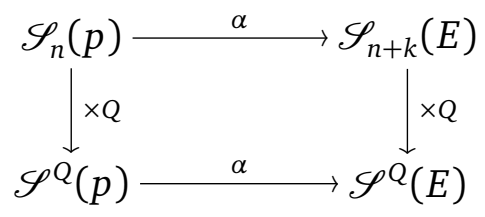


(ii) The collection of maps $(\times Q)$, for $n \in \mathbb{N}$, extend canonically to a map, well-defined up to homotopy,

$$
\times Q: \mathscr{S}(p):=\underset{n \in N}{\operatorname{hocolim}} \mathscr{S}_{n}(p) \rightarrow \mathscr{S}^{Q}(p)
$$

of the infinite mapping telescope of the maps $(\times I)$.

Proof. (i) Use the canonical isomorphism $\alpha(x) \times Q \cong \alpha(x \times Q)$, compare the proof of Remark 4.1.

(ii) We first show that any homeomorphism $f: Q \times I \rightarrow Q$ gives a homotopy in the following diagram:

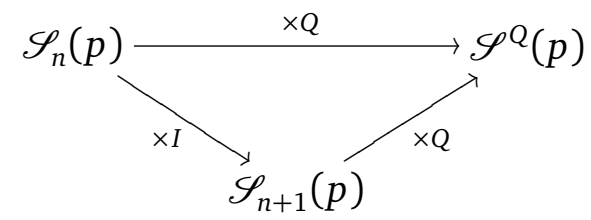

Let $p: E \rightarrow B$, and suppose we are given a bundle $q: E \rightarrow B$ of compact $n$-manifolds together with a fiber homotopy equivalence $E^{\prime} \rightarrow E$, determining an element in $\mathscr{S}_{n}(p)$. Use $f$ to get a bundle isomorphism $E \times Q \times I \rightarrow E \times Q$. Using the Fill-in property, there results a bundle of compact $Q$-manifolds over $B \times I$ which comes with a canonical homotopy equivalence to the fibration $p \times \mathrm{id}_{I}$. This procedure also works for families and produces a map $\mathscr{S}_{n}(p) \rightarrow$ $\mathscr{S}^{\mathrm{Q}}\left(p \times \mathrm{id}_{I}\right)$. Compose with the "evaluation map"

$$
\mathscr{S}^{\mathrm{Q}}\left(p \times \mathrm{id}_{I}\right) \rightarrow \operatorname{map}\left(I, \mathscr{S}^{\mathrm{Q}}(E)\right)
$$

to obtain the desired homotopy.

Therefore a homeomorphism $f: Q \times I \rightarrow Q$ really produces the desired homotopy. If $f$ is isotopic to another homeomorphism $g$, we can use the isotopy to construct a map $\mathscr{S}_{n}(p) \rightarrow \mathscr{S}^{\mathrm{Q}}\left(p \times \mathrm{id}_{I} \times \mathrm{id}_{I}\right)$; composing with the evaluation map then yields a homotopy between $H_{f}$ and $H_{g}$. In this case the two resulting maps hocolim ${ }_{n \in \mathbb{N}} \mathscr{S}_{n}(p) \rightarrow \mathscr{S}^{Q}(p)$ will be homotopic.

Thus, if we want our map $\mathscr{S}(p) \rightarrow \mathscr{S}^{Q}(p)$ to be well-defined up to homotopy, we need to give canonical isotopy class of homeomorphisms $Q \times I \rightarrow Q$. There exists such a canonical isotopy class, for the following reason: The projection $Q \times I \rightarrow Q$ is cell-like, hence a near homeomorphism; on the other hand the space Homeo $(M \times Q, M)$ is locally contractible. Notice that any choice of homeomorphism $M \times Q \rightarrow M$ induces a group structure on $\operatorname{Homeo}(M \times Q, M)$. It follows that $\operatorname{Homeo}(M \times Q, M)$ is even uniformly locally contractible. So 
there is an $\varepsilon>0$ such that any two homeomorphisms are isotopic provided they have distance smaller than $\varepsilon$. Thus we can define the canonical isotopy class of homeomorphisms $Q \times I \rightarrow Q$ to be represented by any homeomorphism which is within an $\frac{\varepsilon}{2}$-ball of the projection.

\subsection{Whitehead torsion on $Q$-manifolds}

The works of Chapman and West show that $Q$-manifold theory is a natural and satisfactory setting for simple homotopy theory: If $f: X \rightarrow Y$ is a map of finite CW complexes, then it is a simple homotopy equivalence if and only if the map $f \times Q: X \times Q \rightarrow Y \times Q$ is homotopic to a homeomorphism ([Cha76, Theorem 38.1]). This allows to define the Whitehead torsion of a homotopy equivalence of $Q$-manifolds $f: M \rightarrow N$ as the torsion of the corresponding map $X \rightarrow Y$, where $X$ and $Y$ are finite CW complexes such that $X \times Q \cong M$, and $Y \times Q \cong N$.

We show in this section that $Q$-manifolds are equally well adapted for higher simple homotopy theory: The parametrized Whitehead torsion map has a version on the level of $Q$-manifolds, which is even a weak homotopy equivalence (contrarily to the finite-dimensional case where tangent bundle data is relevant).

To see this, we need to say more about the proof of Theorem 4.6. The first step in the proof is to define, for an $n$-dimensional euclidean bundle $\gamma$ over $E$, a structure space of $n$-dimensional framed fiber bundle structures $\mathscr{S}_{n}^{f r}(p, \gamma)$ [Hoe09, section 1.2], and to define a corresponding stable version $\mathscr{S}^{f r}(p, \gamma)$.

The homotopy type of this space is independent of $\gamma$, so let us work with $\epsilon$, the trivial euclidean bundle over $E$. The forgetful map $\mathscr{S}^{f r}(p, \epsilon) \rightarrow \mathscr{S}(p)$ is split injective up to homotopy, and [Hoe09, Corollary 1.2.5]

$$
\mathscr{S}(p) \simeq \mathscr{S}^{f r}(p, \epsilon) \times \operatorname{map}(E, B \mathrm{TOP}) .
$$

Theorem 4.6 now follows by showing that the parametrized Whitehead torsion, restricted to framed bundle structures, induces a homotopy equivalence

$$
\tau: \mathscr{S}^{f r}(p, \epsilon) \stackrel{\simeq}{\rightarrow} \Gamma\left(\begin{array}{c}
\Omega \mathrm{Wh}_{B}(E) \\
\downarrow \\
B
\end{array}\right) .
$$

The following result allows to extend the parametrized torsion map to Qmanifolds. 
Lemma 4.14. (i) The composite

$$
\mathscr{S}^{f r}(p, \epsilon) \rightarrow \mathscr{S}(p) \stackrel{\times Q}{\longrightarrow} \mathscr{S}^{Q}(p)
$$

is a homotopy equivalence.

(ii) The triangle

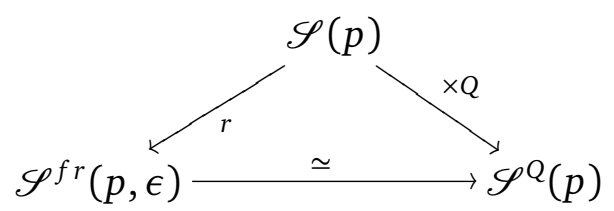

commutes up to homotopy

Before proving this Lemma, let us deduce our main result of this section.

Theorem 4.15. Let $p: E \rightarrow B$ be a bundle of compact Q-manifolds over a compact ANR. There is a diagram, commutative up to homotopy:

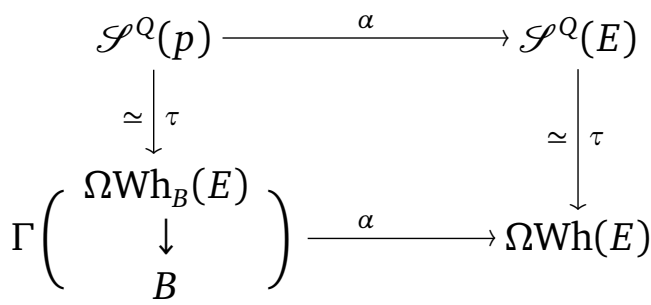

Proof. The map $\tau$ is defined by choosing a homotopy inverse of the map $\mathscr{S}^{f r}(p, \epsilon) \rightarrow \mathscr{S}^{\mathrm{Q}}(p)$ and composing with the characteristic for finite-dimensional manifolds. The the diagram commutes at least if $B$ is a compact topological manifold. To obtain the general case, use the fact that any compact ANR is simple homotopy equivalent to a finite-dimensional compact manifold together with the following Lemma 4.16 .

Lemma 4.16. Let $\varphi: B^{\prime} \rightarrow B$ be a simple homotopy equivalence between compact ANRs. Let $p: E \rightarrow B$ be a fibration, denote by $p^{\prime}: E^{\prime} \rightarrow B^{\prime}$ the fibration pulled back by $\varphi$ and by $\bar{\varphi}: E^{\prime} \rightarrow E$ the structure map. Then the diagram

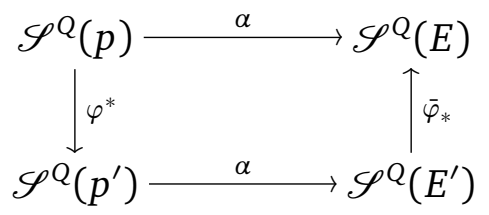

commutes up to homotopy. 
Recall that a homotopy equivalence $\varphi: B^{\prime} \rightarrow B$ between compact ANRs is called a simple homotopy equivalence if the map $\varphi \times \mathrm{id}_{Q}: B^{\prime} \times Q \rightarrow B \times Q$ is homotopic to a homeomorphism. - The proof of the lemma depends on the following result.

Lemma 4.17. For $M$ and $N$ homeomorphic compact Q-manifolds, denote by Homeo $(M, N)$ resp. $C(M, N)$ the space of homeomorphisms resp. continuous maps from $M$ to $N$, with the compact-open topology. If $\overline{\operatorname{Homeo}}(M, N)$ denotes the closure of $\operatorname{Homeo}(M, N)$ in $C(M, N)$, then the inclusion $\operatorname{Homeo}(M, N) \rightarrow$ $\overline{\operatorname{Homeo}}(M, N)$ is a weak homotopy equivalence.

Proof. As shown in the proof of Lemma 4.13, the space $\operatorname{Homeo}(M, N)$ is uniformly locally contractible. It follows from [EW42] that the inclusion $\operatorname{Homeo}(M, N) \rightarrow \overline{\operatorname{Homeo}}(M, N)$ is a weak homotopy equivalence.

Proof of Lemma 4.16. Suppose we can establish the Lemma in the special case $B^{\prime}=B \times I$, and $\varphi$ the projection map, for an arbitrary $B$. Then it is easy to see that, given any $\varphi: B^{\prime} \rightarrow B$, the statement holds for $\varphi$ if and only if it holds for any map homotopic to $\varphi$.

Suppose that, moreover, we can establish the Lemma in the special case $B^{\prime}=B \times Q$, with $\varphi$ again the projection map. Then we conclude that, given any $\varphi: B^{\prime} \rightarrow B$, the statement holds for $\varphi$ if and only if it holds for $\varphi \times \mathrm{id}_{Q}$, which is homotopic to a homeomorphism. But the statement for $\varphi$ a homeomorphism is obviously correct.

So we only need to prove the statement for the cases $B^{\prime}=B \times Q$ (and $\varphi$ the projection map), and $B^{\prime}=B \times I$ (and $\varphi$ the projection map). The proof for both cases is identical and uses the fact that the corresponding map on total spaces is a near homeomorphism. We will give a prove for $B^{\prime}=B \times Q$.

Denote by $q: E \times I \rightarrow I$ the projection. It is enough to construct a simplicial map

$$
H .: \mathscr{S}^{Q}(p) . \rightarrow \mathscr{S}^{Q}(q) .
$$

such that $H$ followed by the restriction onto $\{0\} \subset I$ is $\alpha$, whereas $H$ followed by the restriction onto $\{1\} \subset I$ is given by $\bar{\varphi}_{*} \circ \alpha \circ \varphi^{*}$.

We are going to assign inductively over $n$ to each element $x \in \mathscr{S}^{\mathrm{Q}}(p)_{n}$, represented by a bundle $E^{\prime} \rightarrow \Delta^{n} \times B$, the following data:

(i) A homeomorphism $f(x): E^{\prime} \times Q \rightarrow E^{\prime}$ over $\Delta^{n}$, and

(ii) a homotopy $K(x)$ between $f(x)$ and the projection $p: E^{\prime} \times Q \rightarrow E^{\prime}$ over $\Delta^{n}$. The homotopy is to go through maps in $\overline{\operatorname{Homeo}}\left(E^{\prime} \times Q, E^{\prime}\right)$ and is to be stationary on $\left[1-2^{-n-1}, 1\right]$. 
Both the assignments $x \mapsto f(x)$ and $x \mapsto K(x)$ need to be compatible with the boundary and degeneracy maps. Then $H(x)$ will be given by the bundle $E^{\prime} \times Q \times I \cup_{E^{\prime} \times Q \times\{1\}} E^{\prime}$ over $I \times \Delta^{n}$, together with the map to $E \times I \times \Delta^{n}$ induced by the homotopy $K(x)$ and the map $E^{\prime} \rightarrow E \times \Delta^{n}$ given from $x$.

The induction beginning $n=-1$ is trivial. Suppose that the assignments have been made for $k \leq n-1$. If $x \in \mathscr{S}^{Q}(p)_{n}$ is a degeneracy, then both $f(x)$ and $K(x)$ are the obvious ones. Otherwise, we show that

(i) The homeomorphism

$$
\partial f(x):\left.E^{\prime}\right|_{\partial \Delta^{n}} \times\left. Q \rightarrow E^{\prime}\right|_{\partial \Delta^{n}}
$$

over $\partial \Delta^{n}$, assembled from the $f\left(\partial_{i} x\right)$, extends to a homeomorphism

$$
f(x): E^{\prime} \times Q \rightarrow E^{\prime}
$$

over $\Delta^{n}$.

(ii) The homotopy $\partial K(x)$ between $\partial f(x)$ and the projection $\left.p\right|_{\partial \Delta^{n}}:\left.E^{\prime}\right|_{\partial \Delta^{n}} \times$ $\left.Q \rightarrow E^{\prime}\right|_{\partial \Delta^{n}}$ over $\partial \Delta^{n}$, assembled from the $K\left(\partial_{i} x\right)$, extends to a homotopy $K(x)$ between $f(x)$ and $p$. The homotopy is to go through maps in $\overline{\text { Homeo }}\left(E^{\prime} \times Q, E^{\prime}\right)$ and is to be stationary on $\left[1-2^{-n-1}, 1\right]$.

Notice that the bundle $E^{\prime}$, considered as a bundle over $\Delta^{n}$, is trivial with a fiber we call $M$. Therefore we can reformulate (i) and (ii) in homotopy-theoretic terms as follows: Suppose we are given the solid diagram $\left(c_{p}\right.$ denoting the constant map with value $p$ ):

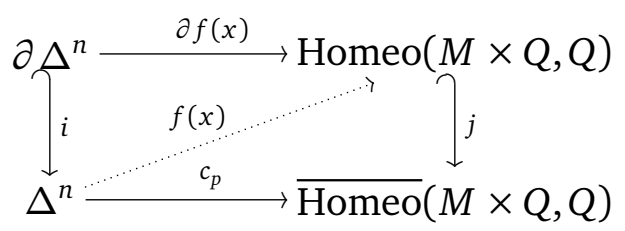

and a homotopy $\partial K(x): \partial \Delta^{n} \times I \rightarrow \overline{\operatorname{Homeo}}(M \times Q, Q)$ between $j \circ \partial f(x)$ and $c_{p} \circ i$, which is stationary on $\left[1-2^{-n}, 1\right]$. Then there is a dotted arrow $f(x)$ such that

(i) $f(x) \circ i=\partial f(x)$, and

(ii) $\partial K(x)$ extends to a homotopy $K(x): \Delta^{n} \times I \rightarrow \overline{\operatorname{Homeo}}(M \times Q, Q)$ between $c_{p}$ and $j \circ f(x)$, which is stationary on $\left[1-2^{-n-1}, 1\right]$.

This homotopy-theoretic problem can always be solved since the inclusion $\operatorname{Homeo}(M \times Q, M) \rightarrow \overline{\operatorname{Homeo}}(M \times Q, M)$ is a weak homotopy equivalence. 
Indeed, first extend the homotopy $\partial K(x)$ to $K^{\prime}: \Delta^{n} \times I \rightarrow \overline{\operatorname{Homeo}}(M \times Q, M)$, stationary on $\left[1-2^{-n}, 1\right]$, and let $c^{\prime}:=K_{1}^{\prime}$. Then, the solid diagram with $c_{p}$ replaced by $c^{\prime}$ commutes strictly, and there is a map $f(x)$ satisfying (i), such that $c^{\prime}$ is homotopic to $j \circ f(x)$ via a homotopy $K^{\prime \prime}$ stationary on $\partial \Delta^{n}$. The homotopy $K(x)$ from $c_{p}$ to $j \circ f(x)$ is then given by $K^{\prime}$ on $\left[0,1-2^{-n}\right]$, by $K^{\prime \prime}$ on $\left[1-2^{-n}, 1-2^{-n-1}\right]$, and by the stationary homotopy on $\left[1-2^{-n-1}, 1\right]$.

This concludes the induction step and therefore the proof.

Now we give a proof of Lemma 4.14 .

Proof of Lemma 4.14. (i) Recall from chapter 2 that $\mathscr{S}_{n}(p)$ is homotopy equivalent to a space of lifts. Similarly,

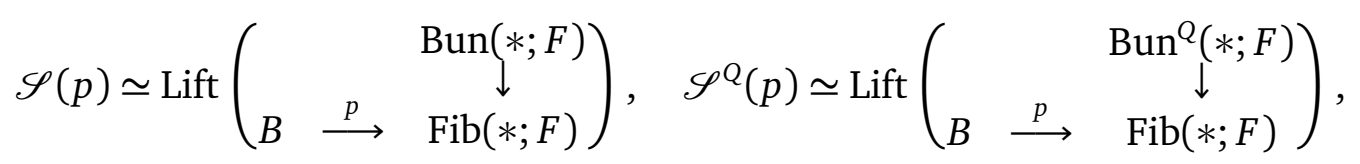

with $\operatorname{Bun}(B ; F)=\operatorname{hocolim}_{n \in \mathbb{N}} \operatorname{Bun}_{n}(B ; F)$, and $\operatorname{Bun}^{Q}(B ; F)$ defined similarly as $\operatorname{Bun}_{n}(B ; F)$ but in terms of $Q$-manifolds.

Denote by $\mathbf{F i b}^{f r}(B ; F)$ the category where an object is a fibration $E \rightarrow B$, with fiber $F_{b}$ over $b$, together with a stable euclidean bundle $\gamma$ over $E$. (Moreover, we assume that the fibration defines an element in $\operatorname{Fib}(B ; F)$ and the bundle is represented by an element of $\operatorname{Bun}_{n}(E ; *)$.) A morphism from $(E \rightarrow B, \gamma)$ to $\left(E^{\prime} \rightarrow B, \gamma^{\prime}\right)$ is a stable bundle map $\gamma \rightarrow \gamma^{\prime}$ such that the underlying map $E \rightarrow E^{\prime}$ is a fiber homotopy equivalence over $B$. The corresponding functor satisfies the Amalgamation, Straightening, and Fill-in property (see section 2) as both bundles and fibrations do. By the same procedure as in section 2, we obtain a simplicial set $\mathrm{Fib}^{f r}(B ; F)$. whose geometric realization we denote by $\mathrm{Fib}^{f r}(B ; F)$, and there is a homotopy equivalence

$$
\mathscr{S}^{f r}(p, \gamma) \rightarrow \operatorname{Lift}\left(\begin{array}{cc} 
& \operatorname{Bun}(* ; F) \\
& \downarrow \\
B \quad \stackrel{(p, \gamma)}{\longrightarrow} & \operatorname{Fib}^{f r}(* ; F)
\end{array}\right) .
$$

Consider the diagram

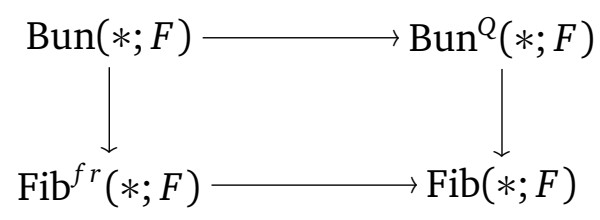


where all the maps are forgetful. (The forgetful map $\operatorname{Bun}(* ; F) \rightarrow \operatorname{Fib}^{f r}(* ; F)$ maps $E$ to $(E, T E)$.)

Notice that proving (i) amounts to proving that the square (3) is homotopy cartesian. Notice as well that $\pi_{0}$ of both vertical fibers is the Whitehead group Wh $(\pi F)$, on the left-hand side by Hoehn's result and on the right-hand side by simple-homotopy theory on $Q$-manifolds.

So, to conclude the proof, we need to show that, given any basepoint $M$ in $\operatorname{Bun}(* ; F)$, the diagram (3) is homotopy cartesian after applying the loop functor $\Omega$ (based at the point given by $M$ ).

In section 2 we showed that the spaces in diagram 3 are disjoint unions of suitable classifying spaces of automorphism monoids. So, applying $\Omega$ yields the left-hand square of the following diagram, whose lines are homotopy fibration sequences (see [WW01, p. 171] for the first line):

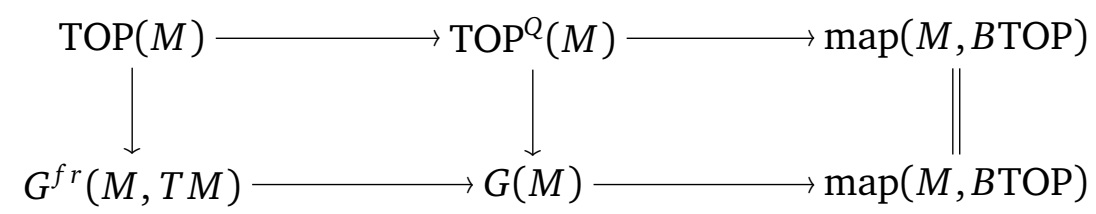

So the horizontal homotopy fibers in diagram (3) agree.

(ii) The retraction $r$ replaces a bundle $E^{\prime} \rightarrow B$ by the composition with an associated disk bundle $b: E^{\prime \prime} \rightarrow E^{\prime}$. As the map $b \times \mathrm{id}_{Q}: E^{\prime \prime} \times Q \rightarrow E^{\prime} \times Q$ is cell-like and therefore a near homeomorphism [Cha76, Theorem 43.1], it induces a path between the bundles $E^{\prime \prime} \times Q \rightarrow B$ and $E^{\prime} \times Q \rightarrow B$ in the space of all bundles. In fact, we may copy the proof of Lemma 4.16 , replacing $E^{\prime} \times Q$ by $E^{\prime \prime} \times Q$, and $E^{\prime}$ by $E^{\prime} \times Q$. 


\section{Applications to fibering questions}

The goal of this section is to apply the results obtained so far in the context of fibering questions. We obtain a complete obstruction theory (Theorems 5.1 and Theorems 5.2) in algebraic $K$-theory for existence and uniqueness of the stable fibering problem: Is a map $f: M \rightarrow B$ between compact topological manifolds homotopic to a fiber bundle, with compact manifolds as fibers, if we allow to stabilize $M$ by crossing with disks of sufficiently high dimension?

In some sense, these results are purely formal consequences of Theorem 4.4, together with the results of [DWW03]. However, for an important special case, namely the "change of total space problem", we also offer a slightly more geometric perspective (see Theorem 5.10), using families of $h$-cobordisms, and the consideration of "thickenings" in the sense of Wall [Wa166], where a stable calculation and a stable range are known. It follows that, whenever a concordance stable range is known for the fibers, we can give an upper estimate on the number of stabilizations needed.

We also give several examples and use a spectral sequence to further analyze our obstructions. This will lead to a comparison with the fibering obstructions of [FLS09] in section 5.8. We are also able to deduce from our general machinery the fibering theorems that Chapman-Ferry proved in the setting of $Q$-manifolds [CF78] (see section 5.7).

Our main interest is the category of topological manifolds - unless stated otherwise, all manifolds will be assumed to be topological manifolds, and the results apply to the topological fibering problem. However the results also apply to the setting of $Q$-manifolds, see section 5.2 . For a discussion of the DIFF case, see Remark 5.5 .

\subsection{Stably fibering manifolds}

Let $f: M \rightarrow B$ a map between compact topological manifolds. We say that $f$ stably fibers if, for some $n \in \mathbb{N}$, the composite

$$
f \circ \text { Proj: } M \times D^{n} \rightarrow M \rightarrow B
$$

is homotopic to the projection map of a fiber bundle whose fibers are compact topological manifolds. In this section we are going to apply our results to the 
following questions:

(i) When does $f$ stably fiber?

(ii) How many different ways do there exist for $f$ to stably fiber? More precisely, denote by $C$ the set of all bundles maps $g: M \times D^{n} \rightarrow B$ for some $n$ which are homotopic to $f \circ$ Proj. We define two elements to be equivalent, $g \sim g^{\prime}$, if, after possibly further stabilization, the two bundles $g$ and $g^{\prime}$ are isomorphic through a homeomorphism $i: M \times D^{N} \rightarrow M \times$ $D^{N}$ (i.e. $i \circ g=g^{\prime}$ ), such that $i$ is homotopic to the identity map. The precise question is then: How can $C / \sim$ be described?

See Remark 5.5 for some comments on the smooth case.

Factor our map $f: M \rightarrow B$ as $p \circ \lambda$, where $p: E \rightarrow B$ is a fibration and $\lambda: M \rightarrow E$ is a homotopy equivalence.

Here are the main results of this section. For more details and explanations, see the remark below.

Theorem 5.1 (Existence). A map $f: M \rightarrow B$ between compact topological manifolds stably fibers if and only if

(i) the fibers of $p$ are homotopy finitely dominated,

(ii) the Wall obstruction Wall $(p) \in H^{0}\left(B ; \mathrm{Wh}\left(F_{b}\right)\right)$ vanishes, and

(iii) the torsion obstruction $o(f)$ in the cokernel of

$$
\pi_{0}(\alpha): H^{0}\left(B ; \Omega \mathrm{Wh}\left(F_{b}\right)\right) \rightarrow \mathrm{Wh}(\pi M)
$$

is zero.

Theorem 5.2 (Classification). There is a bijection from $C / \sim$ to the kernel of the map $\alpha: H^{0}\left(B ; \Omega \mathrm{Wh}\left(F_{b}\right)\right) \rightarrow \mathrm{Wh}(\pi M)$.

Remarks. (i) Recall that a space $X$ is homotopy finitely dominated if there is a finite CW complex $Y$ together with maps $i: X \rightarrow Y$ and $r: Y \rightarrow X$ such that $r \circ i \simeq \mathrm{id}_{X}$.

(ii) The group $\mathrm{Wh}(\pi X)$ is the direct sum of the Whitehead groups of $\pi_{1}(C)$ for all path components $C$ of $X$.

(iii) $\mathrm{Wh}(X)$ denotes the connective topological Whitehead spectrum. By definition,

$$
\mathbb{H}^{\bullet}\left(B ; \mathrm{Wh}\left(F_{b}\right)\right)=\Gamma\left(\begin{array}{c}
\mathrm{Wh}_{B}(E) \\
\downarrow \\
B
\end{array}\right),
$$


which is weakly homotopy equivalent to the homotopy limit of the functor from the category of simplices of $B$ to spaces, sending a simplex $\sigma$ to $\mathrm{Wh}\left(|\sigma|^{*} E\right)$ (see section 3). Also by definition,

$$
H^{i}\left(B ; \mathrm{Wh}\left(F_{b}\right)\right)=\pi_{-i} \mathbb{H}^{\bullet}\left(B ; \mathrm{Wh}\left(F_{b}\right)\right) .
$$

We think of it as the i-th cohomology of $B$ with twisted coefficients in the Whitehead spectrum of the fibers.

(iv) Wall $(p)$ is the image of the parametrized $A$-theory characteristic

$$
\chi(p) \in \Gamma\left(\begin{array}{c}
A_{B}(E) \\
\downarrow \\
B
\end{array}\right)
$$

under the map induced by the natural transformation $A(X) \rightarrow \mathrm{Wh}(X)$. It can be understood as a parametrized version of the Wall obstruction, and is defined when condition (i) of Theorem 5.1 holds.

(v) As defined in section 4, if $B$ is path-connected, the map $\alpha$ is given by the composition

$$
\begin{aligned}
\mathbb{H}^{\bullet}\left(B ; \Omega \mathrm{Wh}\left(F_{b}\right)\right) \rightarrow \mathbb{H}^{\bullet}\left(\{b\} ; \Omega \mathrm{Wh}\left(F_{b}\right)\right) & \simeq \Omega \mathrm{Wh}\left(F_{b}\right) \\
& \stackrel{\chi_{e}(B) \cdot i_{*}}{\longrightarrow} \Omega \mathrm{Wh}(E) \simeq \Omega \mathrm{Wh}(M),
\end{aligned}
$$

the first map being the restriction to some $b \in B$, the labeled map induced by the inclusion, followed by multiplication with the Euler characteristic of $B$. If $B$ is not path-connected, $\alpha$ is defined component-wise.

(vi) We will see that if the first two conditions of Theorem 5.1 are satisfied, then $p$ is fiber homotopy equivalent to a bundle of compact manifolds. Under these hypotheses, the torsion obstruction is defined as follows:

Choose a factorization $f=q \circ \lambda^{\prime}$ such that $q: E^{\prime} \rightarrow B$ is a bundle of compact manifolds and $\lambda^{\prime}$ is a homotopy equivalence. Calculate the Whitehead torsion of the homotopy equivalence $\lambda^{\prime}: M \rightarrow E^{\prime}$, and transport it back to Wh $(\pi M)$ using $\lambda^{\prime}$. Its class in the cokernel of $\pi_{0}(\alpha)$ is, by definition, the torsion obstruction $o(f)$. It is independent of the choice of the bundle $q^{\prime}$. 
Recall from section 4 the geometric assembly map

$$
\alpha: \mathscr{S}_{k}(p) \rightarrow \mathscr{S}_{n+k}(E)
$$

A point in its domain can be understood as a fiber homotopy equivalence $q \rightarrow p$, where $q$ is a bundle of compact topological $k$-manifolds. The image of this point under $\alpha$ is basically the induced homotopy equivalence on the level of total spaces.

Here is the key observation that connects the fibering question with the geometric assembly map.

Lemma 5.3. (i) The fibration $p$ is fiber homotopy equivalent to a bundle of compact topological $k$-manifolds if and only if $\mathscr{S}_{k}(p)$ is non empty.

(ii) A map $f: M^{n+k} \rightarrow B^{n}$ is homotopic to a bundle of $k$-manifolds if and only if the element defined by $\lambda: M \rightarrow E$ is in the image of the map $\pi_{0}(\alpha): \pi_{0} \mathscr{S}_{k}(p) \rightarrow \pi_{0} \mathscr{S}_{n+k}(E)$.

(iii) There is a bijection from $C / \sim$ to the preimage of $[\lambda]$ under the map $\alpha: \pi_{0} \mathscr{S}_{k}(p) \rightarrow \pi_{0} \mathscr{S}_{n+k}(E)$.

Proof. (i) is true by definition, and (ii) follows from (iii). Statement (iii) is basically a close examination of the definition.

Indeed, as $\mathscr{S}_{k}(p)$. is Kan, an element in the preimage of [ $\left.\lambda\right]$ under $\pi_{0}(\alpha)$ is given by a commutative diagram

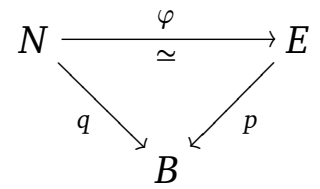

with $q$ a bundle of compact $k$-manifolds, such that $N$ is homeomorphic to $M$ via a map under which $\varphi$ corresponds to $\lambda$ up to homotopy. It defines the same element as the diagram

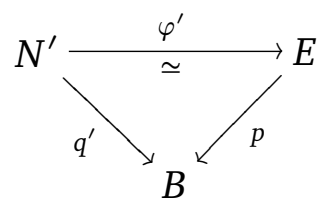


if and only if both elements form are the boundaries of a similar diagram over $B \times I$. This means that both diagrams extend to a diagram

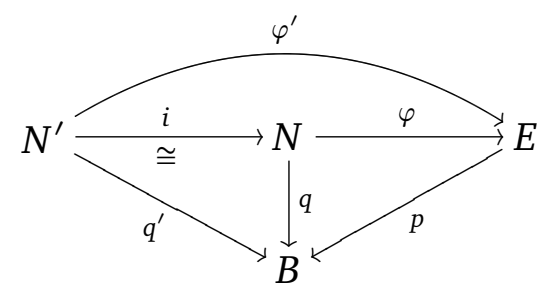

with $i$ a homeomorphism of bundles over $B$, such that the lower triangles commute strictly and the upper triangle commutes up to a homotopy over $B$.

Suppose that $f$ is homotopic to a bundle $g$ of $k$-manifolds, via a homotopy $H$. Then $H$ induces a fiber homotopy equivalence $\varphi: M \rightarrow E$ from $g$ to $p$ together with a homotopy from $\varphi$ to $\lambda$. Setting in the diagram (1) $N:=M$ and $q:=g$ we obtain a corresponding element in the preimage of $[\lambda]$ under $\alpha$. Letting $C^{\prime}:=\{(g, H)\}$ the set of all homotopies $H$ from $f$ to a bundle $g$, we therefore have constructed a map

$$
c: C^{\prime} \rightarrow \pi_{0}(\alpha)^{-1}([\lambda]) .
$$

Suppose now that the images of $(g, H)$ and $\left(g^{\prime}, H^{\prime}\right)$ under $c$ are the same. This means that we obtain a diagram

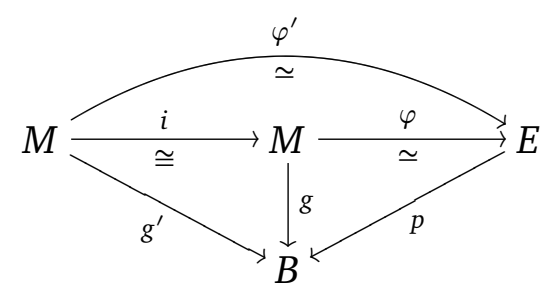

together with a homotopy over $B$ making the upper triangle commute. Therefore

- $g \circ i=g^{\prime}$ (since the triangle formed by these maps commutes strictly), and

- $i$ is homotopic to the identity map (since $\varphi$ and $\varphi^{\prime}$ are both homotopic to $\lambda$ ).

But this means exactly that $g \sim g^{\prime}$ as elements of $C$. Therefore $c$ induces an injection $C / \sim \rightarrow \pi_{0}(\alpha)^{-1}([\lambda])$. 
Finally we need to see that the map $c$ is surjective. Therefore consider an element $x \in \pi_{0} \mathscr{S}_{k}(p)$, represented by a diagram (1). If $\pi_{0}(\alpha)(x)=[\lambda]$, we can extend it to a diagram

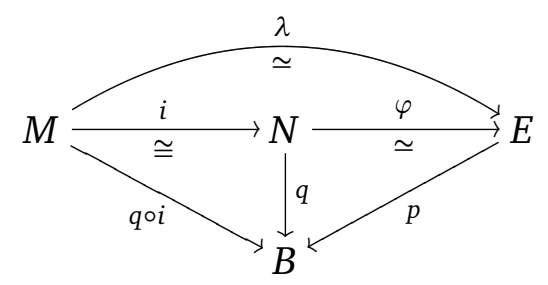

such that the upper triangle commutes up to a homotopy (not necessarily over $B$ ). Without the curved arrow, and with the middle column deleted, this diagram represents $c(q \circ i, H)$ for some homotopy $H$. This shows that our original element is in the same path component as $c(q \circ i, H)$.

Here is a stabilized version of Lemma 5.4, It follows from Lemma 5.4 together with the fact that

$$
\underset{n}{\operatorname{colim}} \pi_{0} \mathscr{S}_{n}(p) \stackrel{\cong}{\rightarrow} \pi_{0} \underset{n}{\operatorname{hocolim}} \mathscr{S}_{n}(p) .
$$

Lemma 5.4. (i) The fibration $p$ is fiber homotopy equivalent to a bundle of compact topological manifolds if and only if $\mathscr{S}(p)$ is non empty.

(ii) A map $f: M \rightarrow B$ stably fibers if and only if the element defined by $\lambda: M \rightarrow$ $E$ is in the image of the map $\pi_{0}(\alpha): \pi_{0} \mathscr{S}(p) \rightarrow \pi_{0} \mathscr{S}(E)$.

(iii) There is a bijection from $C / \sim$ to the preimage of $[\lambda]$ under the map $\alpha: \pi_{0} \mathscr{S}(p) \rightarrow \pi_{0} \mathscr{S}(E)$.

Proof of Theorem 5.1. We first show that assumptions (i) and (ii) are necessary. In fact, if $f$ is homotopic to a bundle $g$ of compact topological manifolds, then assumptions (i) clearly holds.

Moreover, there is a weak homotopy fibration sequence

$$
\Gamma\left(\begin{array}{c}
A_{B}^{\%}(E) \\
\downarrow \\
B
\end{array}\right) \rightarrow \Gamma\left(\begin{array}{c}
A_{B}(E) \\
\downarrow \\
B
\end{array}\right) \rightarrow \Gamma\left(\begin{array}{c}
\mathrm{Wh}_{B}(E) \\
\downarrow \\
B
\end{array}\right) .
$$

So Wall $(p)$ is zero if and only if $\chi(p)$ lifts over the fiberwise assembly map, up to homotopy. By [DWW03] this is equivalent to saying that the fibration $p$ is fiber homotopy equivalent to a bundle of compact topological manifolds. 
Now the homotopy from $f$ to $g$ induces a fiber homotopy equivalence from $p$ to $g$, so Wall $(p)=0$. So assumption (ii) is also necessary.

Now suppose that assumption (i) and (ii) hold, such that we can factor $f=q \circ \lambda^{\prime}$ where $q$ is a fiber bundle of compact topological manifolds and $\lambda^{\prime}$ a homotopy equivalence. Denote by $F_{b}^{\prime}$ the fiber of $q$ over $b$ and consider the following commutative diagram

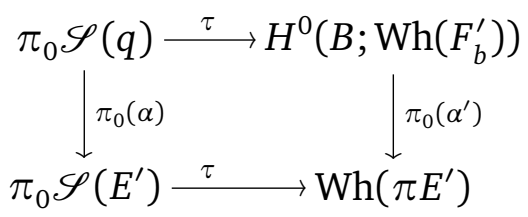

which is $\pi_{0}$ of the pull-back square from Theorem 4.4.

By Lemma 5.4, $f$ is homotopic to a bundle of compact manifolds if and only if the element defined by $\lambda^{\prime}$ in the lower left-hand corner comes from an element in the upper left-hand corner. Using the pull-back property, this is equivalent to saying that the corresponding element $\tau\left(\left[\lambda^{\prime}\right]\right)$ in the lower right-hand corner comes from an element in the upper right-hand corner. Thus, if we define $o(f)$ as the class of $\tau\left(\left[\lambda^{\prime}\right]\right)$ in the cokernel of $\alpha^{\prime}, f$ fibers stably if and only if $o(f)=0$. As the fibrations $p$ and $q$ are fiber homotopy equivalent, $\lambda^{\prime}$ induces a bijection between the cokernels of $\pi_{0}(\alpha)$ and $\pi_{0}\left(\alpha^{\prime}\right)$. So we may think of $o(f)$ as an element in the cokernel of $\pi_{0}(\alpha)$.

Finally we have to show that $o(f)$ is well-defined. Indeed suppose that we choose another factorization $f=\bar{q} \circ \bar{\lambda}^{\prime}$ with $\bar{q}: \bar{E}^{\prime} \rightarrow B$ a fiber bundle of compact manifolds. Then by the composition rule the resulting torsion changes by the torsion of $\lambda^{\prime} \circ \bar{\lambda}^{\prime-1}: \bar{E}^{\prime} \rightarrow E^{\prime}$, which is in the image of $\alpha$ since it comes from a fiber homotopy equivalence. Thus, when passing to the cokernel of $\pi_{0}(\alpha)$, the element $o(f)$ is not affected.

Proof of Theorem 5.2 We saw in Lemma 5.4 that $C / \sim$ is in bijection with $\pi_{0}(\alpha)^{-1}([\lambda])$, which by square (2) is in bijection to $\pi_{0}\left(\alpha^{\prime}\right)[\tau(\lambda)]$ and thus to the kernel of $\pi_{0}\left(\alpha^{\prime}\right)$ as $\alpha^{\prime}$ is an infinite loop map. Now use that $\lambda^{\prime}$ induces a bijection from the kernel of $\pi_{0}(\alpha)$ to $\pi_{0}\left(\alpha^{\prime}\right)$.

Remark 5.5. As discussed in Remark 4.11, a proof of the DIFF analogues of our results seems to require a different approach to the Dwyer-Weiss-Williams characteristics. However the mere $\pi_{0}$-commutativity of the square in Theorem 4.4 only uses additivity and product rule for the classical Whitehead torsion and therefore clearly holds for the corresponding DIFF square. The methods of this work therefore show: The DIFF analogues of our fibering obstructions 
Wall $(p)$ and $o(f)$ (using the DIFF Whitehead space throughout) are defined, and their vanishing is a necessary condition to stably DIFF fibering.

\subsection{Fibering $Q$-manifolds over compact ANRs}

Let $M$ be a compact $Q$-manifold, $B$ a compact ANR, and $f: M \rightarrow B$ a map. This section deals with fibering $M$ over $B$ :

(i) When is $f$ homotopic to a bundle of compact $Q$-manifolds? (By a bundle of $Q$-manifolds, we mean a fiber bundle whose fibers are compact $Q$ manifolds.)

(ii) What is the classification of bundles $g: M \rightarrow B$ sucht that $g \simeq f$ ? More precisely, denote by $C$ the set of all such bundles $g$. We define two elements to be equivalent, $g \sim g^{\prime}$, if the two bundles $g$ and $g^{\prime}$ are isomorphic through a homeomorphism $i: M \rightarrow M$ (i.e. $i \circ g=g^{\prime}$ ), such that $i$ is homotopic to the identity map. How can $C / \sim$ be described?

The main result of this section is that the obstruction theory for stably fibering finite-dimensional manifolds applies to fibering $Q$-manifolds. As in the last section, factor our map $f$ into a homotopy equivalence $\lambda$ and a fibration $p$.

Theorem 5.6 (Existence). Let $M$ be a compact Q-manifold, B a compact ANR. A map $f: M \rightarrow B$ is homotopic to a bundle of compact Q-manifolds if and only if

(i) the fibers of $p$ are homotopy finitely dominated,

(ii) the Wall obstruction Wall $(p) \in H^{0}\left(B ; \mathrm{Wh}\left(F_{b}\right)\right)$ vanishes, and

(iii) the torsion obstruction $o(f)$ in the cokernel of

$$
\alpha: H^{0}\left(B ; \Omega \mathrm{Wh}\left(F_{b}\right)\right) \rightarrow \mathrm{Wh}(\pi M)
$$

is zero.

Theorem 5.7 (Classification). There is a bijection from $C / \sim$ to the kernel of the map $\alpha: H^{0}\left(B ; \Omega \mathrm{Wh}\left(F_{b}\right)\right) \rightarrow \mathrm{Wh}(\pi M)$.

The proof of Theorem 5.6 and 5.7 are similar to the finite-dimensional versions. Use the fact that Lemma 5.3 works for any kind of bundles, and replace Theorem 4.4 by Corollary 4.15 . 


\subsection{Change of base and total space}

The two problems of "change of base" and "change of total space" are interesting special cases where the parametrized Wall obstruction does not play a role. We first consider them in the light of the general theory. After that we offer a second, more geometric perspective using families of $h$-cobordisms. This second perspective makes it easier to find an estimate for a stable range.

The following two theorems also apply in the setting of $Q$-manifolds.

Theorem 5.8 (Change of total space). Let $p: M \rightarrow B$ be a fiber bundle of compact topological manifolds over a compact topological manifold, and let $N$ be another compact topological manifold, equipped with a homotopy equivalence $f: N \rightarrow M:$

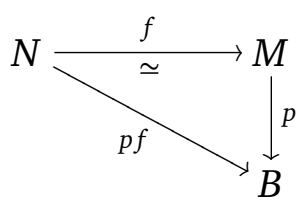

Then $p f$ stably fibers if and only if the Whitehead torsion $\tau(f)$ lies in the image of

$$
\pi_{0}(\alpha): H^{0}\left(B ; \Omega \mathrm{Wh}\left(F_{b}\right)\right) \rightarrow \mathrm{Wh}(\pi M)
$$

for $p$.

Theorem 5.9 (Change of base). Let $p: M \rightarrow B$ be a fiber bundle of compact topological manifolds over a compact topological manifold, and let $C$ be another compact topological manifold, equipped with a homotopy equivalence $f: B \rightarrow C$ :

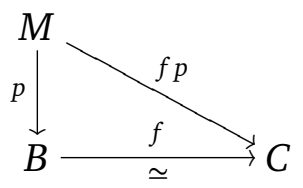

Then $f p$ stably fibers if and only if the image of the Whitehead torsion $f_{*}^{-1} \tau(f) \in$ $\mathrm{Wh}(\pi B)$ under the transfer homomorphism

$$
p^{*}: \mathrm{Wh}(\pi B) \rightarrow \mathrm{Wh}(\pi M)
$$

lies in the image of $\pi_{0}(\alpha)$.

In particular, if the fiber $F$ of $p$ is connected, $\pi_{1}(B)$ acts trivially on $F$, and fp stably fibers, then

$$
\chi_{e}(F) \cdot \tau(f)=0 \in \mathrm{Wh}(\pi C)
$$


Proof of Theorem 5.8. Notice that $p f$ is already a factorization into a homotopy equivalence followed by a fiber bundle. So, conditions (i) and (ii) of Theorem 5.1 are satisfied, and the torsion obstruction $o(f \circ g)$ is just the image of the Whitehead torsion of $f$ in the cokernel.

Proof of Theorem 5.9. Denote by $k: C \rightarrow B$ a homotopy inverse of $f$, and consider the pull-back

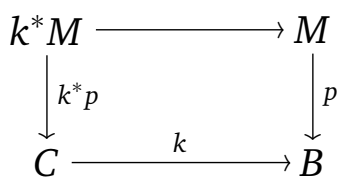

Now $f$ induces a map $\bar{f}: M \rightarrow k^{*} M$ such that $f \circ p=k^{*} p \circ \bar{f}$ is a factorization of $f \circ p$ into a homotopy equivalence followed by a fiber bundle. Thus $o(f \circ p)$ is given by the class of $\bar{f}_{*}^{-1} \tau(\bar{f})$, which satisfies

$$
\bar{f}_{*}^{-1} \tau(\bar{f})=p^{*} f_{*}^{-1} \tau(f)
$$

by the geometric definition of the transfer map [And74].

Now suppose that $F$ is connected and $\pi_{1}(B)$ acts trivially. In this case the composite $p_{*} \circ p^{*}$ is just multiplication with the Euler characteristic of $F$ [Lüc87]. We saw that if $f \circ p$ stably fibers, then $p^{*} f_{*}^{-1} \tau(f)$ comes from some element $\kappa \in \mathrm{Wh}(\pi F)$ under the map induced by the inclusion $i: F \rightarrow M$. As the composite $p \circ i$ is nullhomotopic, we have

$$
0=p_{*} i_{*} \kappa=p_{*} p^{*} f_{*}^{-1} \tau(f)=\chi_{e}(F) \cdot f_{*}^{-1} \tau(f) \in \mathrm{Wh}(\pi B) .
$$

Using the relation between the parametrized torsion and higher $h$-cobordism theory, we now give a second perspective on the change of total space problem. Under smoothability conditions, this approach allows to estimate a stable range using the stability results of Igusa [Igu88].

In the change of total space problem as in Theorem 5.8 (i), suppose for simplicity that $B$ is connected. Denote by $k$ the smallest dimension of a CW complex homotopy equivalent to $B$, and by $n$ the the smallest dimension of a CW complex homotopy equivalent to the fibers. 
Theorem 5.10. In the situation of Theorem 5.8 suppose that $M, N$, and the fibers of $f$ are smoothable. If $\tau(f)$ is in the image of $\pi_{0}(\alpha)$, then the composite

$$
\bar{N}:=N \times I \stackrel{\text { Proj }}{\longrightarrow} N \stackrel{f}{\rightarrow} M \stackrel{p}{\rightarrow} B
$$

fibers as soon as

$$
\begin{aligned}
\operatorname{dim} \bar{N} \geq \max \{2(n+k)+1, & \operatorname{dim} M+n+k, \operatorname{dim} M+k+2, \\
& \operatorname{dim} B+2 k+6, \operatorname{dim} B+3 k+2, \operatorname{dim} N+3\} .
\end{aligned}
$$

Proof. The first step is to replace $f: N \rightarrow M$ by $\bar{f}: N \rightarrow \bar{M}$ which is a stably tangential homotopy equivalence, i.e. $\bar{f}^{*} T \bar{M} \cong T N$ stably.

Therefore recall that $M$ and $N$ are supposed to be smoothable, so we may consider a vector bundle reduction

$$
\left(f^{-1}\right)^{*} T N-T M: M \rightarrow B O
$$

of the corresponding TOP bundle. It actually has a further reduction to a $O(n+k)$-bundle, the inclusion $B O(N) \rightarrow B O$ being $N$-connected. Let therefore $q: \bar{M} \rightarrow M$ be a disk bundle of this $(n+k)$-dimensional vector bundle. We obtain

$$
\left.\left.T \bar{M}\right|_{M} \cong T M \oplus T_{\text {fib }} q\right|_{M} \cong T M \oplus q \cong\left(f^{-1}\right)^{*} T N
$$

stably, so if we let $\bar{f}: N \rightarrow \bar{M}$ be $f$ followed by the zero-section, then $\bar{f}$ is a stable tangential homotopy equivalence.

Notice that the map $\bar{p}=p \circ q$ still is a fiber bundle of compact smoothable manifolds (with a fiber we denote by $\bar{F}_{b}$ ), and that

$$
\operatorname{dim} \bar{M}=\operatorname{dim} M+(n+k) .
$$

Now suppose that $\tau(\bar{f})=\tau(f)$ disassembles, i.e. that there is a $\tau \in$ $H^{0}\left(B ; \Omega \mathrm{Wh}\left(\bar{F}_{b}\right)\right)$ such that that $\pi_{0}(\alpha)(\tau)=\tau(f)$. In fact, we would like to further go back to $H^{0}\left(B ; \Omega W h\left(\partial \bar{F}_{b}\right)\right)$. As a first step, the following lemma shows that the inclusion $\partial \bar{F}_{b} \subset \bar{F}_{b}$ is $(n+k-1)$-connected.

Lemma 5.11. If $\bar{F} \rightarrow F$ is a $K$-disk bundle over a compact manifold, and the pair $(F, \partial F)$ is $N$-connected, then $(\bar{F}, \partial \bar{F})$ is $(N+K)$-connected.

As $K$-theory preserves connectivity, we have that $\Omega \mathrm{Wh}\left(\partial \bar{F}_{b}\right) \rightarrow \Omega \mathrm{Wh}\left(\bar{F}_{b}\right)$ is $(n+k-2)$-connected. Using a spectral sequence argument (see section 5.6), we see that the inclusion-induced map

$$
i: H^{0}\left(B ; \Omega \mathrm{Wh}\left(\partial \bar{F}_{b}\right)\right) \rightarrow H^{0}\left(B ; \Omega \mathrm{Wh}\left(\bar{F}_{b}\right)\right)
$$


is $(n-2)$-connected. Hence, stabilizing twice if necessary, we find an element $\tau^{\prime}$ in the left-hand side which maps to $\tau$ under $i$. Let $\partial_{\text {fib }} \bar{p}$ be the restriction of $\bar{p}$ along the fiberwise boundary. Using our generalization of Waldhausen's map

$$
W: \pi_{0} \mathscr{H}\left(\partial_{\mathrm{fib}} \bar{p}\right) \rightarrow H^{0}\left(B ; \Omega \mathrm{Wh}\left(\partial_{\mathrm{fib}} \bar{F}_{b}\right)\right)
$$

from section 3.7 together with the connectivity result Corollary 3.19, we see that this map is surjective provided $k-1$ is in the concordance stable range for $\partial \bar{F}_{b}$.

By our assumptions the manifold $\partial \bar{F}_{b}$ is smoothable, so Igusa's stability result [Igu88] (see [WW01, Theorem 1.3.4] for the topological range) says that $k-1$ is in the concordance stable range for $\partial \bar{F}_{b}$ whenever

$$
\operatorname{dim} \partial \bar{F}_{b} \geq \max \{(2(k-1)+7,3(k-1)+4\} .
$$

Thus, stabilizing further if necessary, we obtain a parametrized $h$-cobordism $E$ over $\partial_{\text {fib }} \bar{p}$ whose torsion equals $-\tau^{\prime}$. Now $\bar{M}$ has dimension

$$
\max \{\operatorname{dim} M+n+k, \operatorname{dim} M+k+2, \operatorname{dim} B+2 k+6, \operatorname{dim} B+3 k+2\} .
$$

Form the bundle $\overline{\bar{M}}:=\bar{M} \cup_{\partial_{\mathrm{fib}} \bar{p}} E$ over $B$. Using additivity for the Whitehead torsion, we see that the inclusion $\bar{M} \rightarrow \overline{\bar{M}}$ has has Whitehead torsion $-\tau(f)$. By the composition rule, the composite

$$
\overline{\bar{f}}: N \rightarrow \bar{M} \rightarrow \overline{\bar{M}}
$$

has torsion zero (and is still stably tangential).

Now stabilize $N$ to obtain $\bar{N}:=N \times I^{l}$ such that $l \geq 3$ (so that $\bar{N}$ is $\pi-\pi$ ) and $\operatorname{dim} \bar{N} \geq 2(n+k)+1$, and stabilize either $\bar{N}$ or $\overline{\bar{M}}$ further so that the dimensions agree. Letting $K$ be a finite $(n+k)$-dimensional CW complex simple homotopy equivalent to $\bar{N}$, it follows that both $\bar{N}$ and $\overline{\bar{M}}$ define thickenings of $K$ in the sense of Wall [Wal66]. It is known that stably, thickenings are classified by their tangent bundle. Now the dimension of the thickenings we consider exceeds $2(n+k)$, so we are in the stable range. But $\overline{\bar{g}}$ is stably tangential, hence the thickenings agree. Thus $\overline{\bar{g}}$ is homotopic to a homeomorphism.

Summarizing all the necessary stabilizations, we see that $p f$ fibers as soon as

$$
\begin{aligned}
\operatorname{dim} \bar{N} \geq \max \{2(n+k)+1, \operatorname{dim} N+3, \operatorname{dim} M+n+k, \\
\operatorname{dim} M+k+2, \operatorname{dim} B+2 k+6, \operatorname{dim} B+3 k+2\} .
\end{aligned}
$$


Finally we give a proof of Lemma 5.11.

Proof of Lemma 5.11. There is a push-out diagram

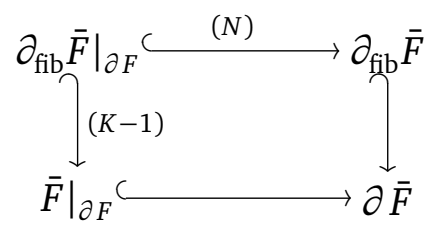

where the labels on the arrows denote the connectivity of the maps. (The left-hand vertical map is $(K-1)$-connected as

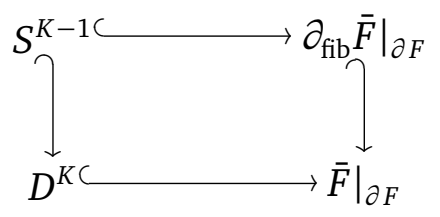

is a homotopy pull-back, with horizontal fibers $\Omega \partial F$.)

We obtain a diagram of pairs

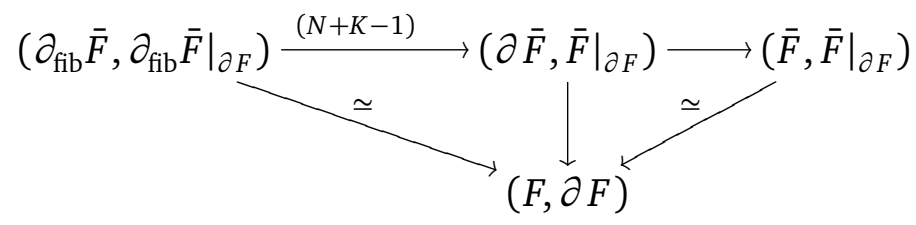

where the left-hand horizontal map is $(N+K-1)$-connected by the theorem of Blakers-Massey, and the left-hand and right-hand diagonal maps are weak equivalences of pairs. Hence the vertical map in the middle is $(N+K)$ connected. Therefore the right-hand horizontal map is $(N+K)$-connected, too.

This implies that the inclusion $\partial \bar{F} \rightarrow \bar{F}$ is also $(N+K)$-connected.

\subsection{Examples I: Elementary applications}

In this section we give some immediate applications of our results on the stable fibering problem. The first one characterizes simple homotopy equivalences between compact manifolds as the homotopy equivalences that stably fiber. After that we give some implications for closed manifolds. 
Proposition 5.12. A homotopy equivalence $f: M \rightarrow N$ between compact manifolds stably fibers if and only if $\tau(f)=0$.

If $M$ and $N$ are closed smoothable of dimension $k$ and $\tau(f)=0$, then $f$ fibers after at most $\max \{2 k+6,3 k+2\}$ stabilizations.

Proof of Proposition 5.12 This is a simple application of our results in the following change of total space problem:

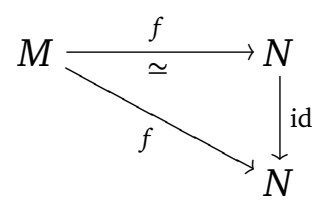

As the fibers of the identity are contractible, their Whitehead group vanishes. So $\pi_{0}(\alpha)$ is the zero map and its cokernel is just $\mathrm{Wh}(\pi M)$. Hence $o(f)=$ $f_{*}^{-1} \tau(f) \in \mathrm{Wh}(\pi M)$.

Now we turn to closed manifolds and consider the change of total space problem

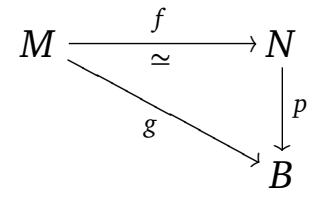

If $g$ stably fibers, i.e. for some $n \gg 0, M \times D^{n+1} \rightarrow B$ fibers, then we may restrict to the boundary to see that

$$
\bar{g}: M \times S^{n} \rightarrow M \stackrel{g}{\rightarrow} B
$$

fibers for large enough $n$. So our theory gives sufficient conditions for $M \times S^{n}$ to fiber over $B$.

On the other hand, if $\bar{g}$ fibers, then it certainly stably fibers. It follows:

Proposition 5.13. (i) A necessary condition for $M \times S^{2 N} \rightarrow B$ to fiber for large $N$ is that

$$
2 o(g)=0 \text {. }
$$

(ii) A sufficient condition for $M \times S^{2 N} \rightarrow B$ to fiber for large $N$ is that

$$
o(g)=0 \text {. }
$$

(iii) The sufficient condition is not necessary in general. 
Proof. (i) We have $\tau\left(f \times \mathrm{id}_{S^{2 N}}\right)=\chi\left(S^{2 N}\right) \cdot \tau(f)=2 \tau(f)$, and its class in the cokernel of $\pi_{0}(\alpha)$ defines the obstruction $o(\bar{g})$ for $\bar{g}$ to stably fiber.

(ii) Stabilize $g$ and restrict to the boundary.

(iii) Choose a homotopy equivalence $f: M \rightarrow K \times S^{1}$ between closed manifolds such that $\tau(f) \neq 0$ and $2 \tau(f)=0$, and let $p: K \times S^{1} \rightarrow B$ denote the projection. Hence $o(p f) \neq 0$; in contrast, if $q: M \times S^{2 N} \rightarrow M$ is the projection, then $o(p f q)=0$.

We will show in Theorem 5.25 that for $B=S^{1}$, the stable fibering obstruction $o(p f q)$ and the obstructions $\tau_{\text {fib }}(p f q)$ defined in [FLS09] agree. Now Farrell's fibering theorem together with the comparison of the different obstructions [FLS09, Theorem 8.1] shows that $p f q$ fibers.

Proposition 5.14. If $f: M \rightarrow B$ is any map between closed manifolds whose homotopy fiber is finitely dominated, then the composite

$$
M \times S^{1} \times S^{N} \stackrel{\text { Proj }}{\longrightarrow} M \stackrel{f}{\rightarrow} B
$$

fibers for large enough $N$.

Proof. The parametrized Wall obstruction becomes zero after crossing with $S^{1}$ (see e.g. [WW01, Corollary 5.2.5]). The same is true for the fibering obstruction. Therefore $M \times S^{1} \times D^{N+1} \rightarrow B$ fibers. Now restrict to the boundary.

\subsection{Examples II: Stable vs. unstable fibering and TOP vs. DIFF}

In this section we give examples of maps $f: M \rightarrow B$ that fiber stably but not unstably. Of course, if the dimension of $M$ and $B$ agree then fibering $f$ unstably just means deforming the map to a homeomorphism, whereas $f$ stably fibers if and only if it is a simple homotopy equivalence (Proposition 5.12): This gives obvious examples.

More interestingly, we will produce two types of examples of arbitrarily high codimension. The first one uses bundle theory and characteristic classes while the second one applies surgery theory (and actually produces an example which does not even block fiber).

At the end we use an example of Klein-Williams to produce maps that fiber in TOP but do not fiber in DIFF. 


\section{Bundle theory}

Let $Z$ be an exotic complex projective space equipped with a homotopy equivalence

$$
h: Z \rightarrow \mathbb{C} P^{2 n+1}
$$

such that, for some $k \neq 0$, the $\mathscr{L}$-genus satisfies

$$
\left(h^{*}\right)^{-1} \mathscr{L}(Z)=\mathscr{L}\left(\mathbb{C} P^{2 n+1}\right) \cdot\left(1+8 k e^{2 n}\right) \in H^{*}\left(\mathbb{C} P^{2 n+1}\right) \cong \mathbb{Q}[e] /\left(e^{2 n+2}\right) .
$$

(Take all the cohomology rings with rational coefficients.) We will show below that such objects exist.

Proposition 5.15. (i) The composite

$$
Z \times S^{N} \stackrel{\pi}{\rightarrow} Z \stackrel{h}{\rightarrow} \mathbb{C} P^{2 n+1}
$$

of $h$ with the projection fibers stably. If $Z$ is smoothable then it fibers even unstably whenever $N \geq 12 n+7$.

(ii) For $N \leq 2 n-1$, the map from (ii) does not fiber (unstably).

Proof. (i) This is an application of the change of total space problem.

(ii) Let $p: Z \times S^{N} \rightarrow \mathbb{C} P^{2 n+1}$ be a fiber bundle homotopic to the map of (ii). Then

$$
T\left(Z \times S^{N}\right) \cong p^{*} T\left(\mathbb{C} P^{2 n+1}\right) \oplus \eta
$$

for an $N$-dimensional bundle $\eta$. Hence

$$
\mathscr{L}\left(Z \times S^{N}\right)=p^{*} \mathscr{L}\left(\mathbb{C} P^{2 n+1}\right) \cdot \mathscr{L}(\eta) .
$$

But $\mathscr{L}\left(Z \times S^{N}\right)=\pi^{*} \mathscr{L}(Z)=p^{*}\left(h^{*}\right)^{-1} \mathscr{L}(Z)$ as the sphere is stably parallelizable. It follows that

$$
\mathscr{L}(\eta)=p^{*}\left(\mathscr{L}\left(\mathbb{C} P^{2 n+1}\right)^{-1} \cdot\left(h^{*}\right)^{-1} \mathscr{L}(Z)\right)=1+8 k p^{*}(e)^{2 n}
$$

using (3) for the last equality. Hence, since $p^{*}$ is injective, the $\mathscr{L}$-genus of $\eta$ is non-zero in degree $4 n$. Inductively one concludes

$$
p_{i}(\eta)=0(i<n), \quad p_{n}(\eta) \neq 0
$$

using the fact that the coefficient of $p_{i}$ in $\mathscr{L}_{i}$ is non-zero for all $i$ [Hir78, I.1.(11)]. So $\eta$ must be at least $2 n$-dimensional: $N \geq 2 n$. 
We now indicate why a homotopy equivalence

$$
h: Z \rightarrow \mathbb{C} P^{2 n+1}
$$

with the property (3) exists. This construction is due to Madsen-Milgram [MM79]. Let $f: X \rightarrow \mathbb{C} P^{2 n}$ be a topological degree one normal map corresponding to the composite

$$
\mathbb{C} P^{2 n} \rightarrow \mathbb{C} P^{2 n} / \mathbb{C} P^{2 n-1} \cong S^{4 n} \rightarrow G / \mathrm{TOP}
$$

where the last map represents $k$ times a generator of $\pi_{4 n}(G /$ TOP $) \cong \mathbb{Z}$. Let $E \rightarrow \mathbb{C} P^{2 n}$ be the disk bundle of the tautological vector bundle. We may pull back the normal map $f$ to $E$. By the $\pi-\pi$-theorem, this pulled-back normal map is cobordant to a map $g: Y \rightarrow E$ which is a homotopy equivalence and restricts to a homotopy equivalence over the boundary $\partial E=S^{4 n+1}$. The Poincaré conjecture implies that $\partial Y \cong S^{4 n+1}$ homeomorphically. Thus we may cone off $g$ at the boundaries to obtain a topological manifold $Z$ and a homotopy equivalence $h: Z \rightarrow \mathbb{C} P^{2 n+1}$.

We have to show that (3) holds. To do that, we will use the characteristic classes

$$
K_{4 n} \in H^{4 n}(G / \mathrm{TOP} ; \mathbb{Q})
$$

given uniquely by the property that if $\gamma: M \rightarrow G /$ TOP is a normal invariant on a closed $4 k$-manifold, then its simply-connected surgery obstruction is given by the formula [MM79, Theorem 4.9]

$$
s(M, \gamma)=\left\langle\mathscr{L}(M) \cdot\left(\sum_{i \geq 1} \gamma^{*}\left(K_{4 i}\right)\right),[M]\right\rangle .
$$

Now the surgery obstructions of $\left.h\right|_{\mathbb{C} P^{i}}$ for $i<2 n$ are zero while the surgery obstruction of $\left.h\right|_{\mathbb{C} P^{2 n}}$ is $k$, hence inductively one concludes that

$$
\gamma^{*}\left(K_{4 i}\right)=0 \quad(4 i<2 n), \quad \gamma^{*}\left(K_{4 n}\right)=k e^{2 n} \in H^{4 n}\left(\mathbb{C} P^{2 n+1}\right) .
$$

Denote by $\mathscr{L} \in H^{*}(B \mathrm{TOP} ; \mathbb{Q})$ the $\mathscr{L}$-class of the universal bundle. By [MM79, Corollary 4.22], its restriction along $G / \mathrm{TOP} \rightarrow B$ TOP is given by $1+8 K$, where $K=K_{4}+K_{8}+\ldots$. Hence

$$
\left(h^{*}\right)^{-1} \mathscr{L}(Z)=\mathscr{L}\left(\mathbb{C} P^{2 n+1}\right) \cdot \gamma^{*}(1+8 K)=\mathscr{L}\left(\mathbb{C} P^{2 n+1}\right) \cdot\left(1+8 k e^{2 n}\right),
$$

as claimed. 
Remark 5.16. To the knowledge of the author, it is unknown in general which of these fake $\mathbb{C} P^{2 n+1}$ are smoothable. The following argument shows that there are infinitely many smoothable examples for even $n$. This follows from calculations of Brumfiel's [Bru71]; I am grateful to Diarmuid Crowley for drawing my interest to that paper.

For each $n$ there exists a number $A_{n}$ such that the normal invariant $f$ is smoothable if and only if $k$ is a multiple of $A_{n}$. (In fact $A_{n}$ is the order of the generator of $\pi_{4 n}(G / \mathrm{TOP})$ in the torsion group $\pi_{4 n-1}(\mathrm{TOP} / O)$ ). ) Hence the subgroup of all smooth normal invariants of $\mathbb{C} P^{2 n}$ satisfying (3) is infinite.

Using the $\pi$ - $\pi$-theorem in the smooth setting, we obtain a map

$$
\left[\mathbb{C} P^{2 n}, G / O\right] \cong[E, G / O] \cong \mathscr{S}^{\mathrm{DIFF}}(E) \rightarrow \mathscr{S}^{\mathrm{DIFF}}(\partial E)=\Gamma_{4 n+1}
$$

from the smooth normal invariants of $\mathbb{C} P^{2 n}$ to the group of homotopy spheres. By [Bru71, Corollary 6.6], this map is a group homomorphism if $n$ is even. Hence in this case it has an infinite kernel.

If $f: X \rightarrow \mathbb{C} P^{4 n}$ is a smooth normal map of degree one which represents an element in the kernel, this means the following: The pull-back of $f$ to $E$ is cobordant to a normal map $g: Y \rightarrow E$ which restricts to a diffeomorphism on the boundary. In this case the coning procedure yields a homotopy equivalence $h: Z \rightarrow \mathbb{C} P^{4 n+1}$ where $Z$ is smooth.

\section{Surgery theory}

Now we come to the surgery-theoretic example. Let $\gamma: X \rightarrow G /$ TOP be a normal invariant on a closed manifold $X$, let $M$ be a closed manifold and let $h: M \rightarrow X \times S^{k}$ be a simple homotopy equivalence which, considered as a normal invariant, restricts to $\gamma$ over $X \times\{*\}$. (See below for an argument that such a homotopy equivalence always exists provided $k \geq 3$ and $k+\operatorname{dim} X \geq 6$.)

Proposition 5.17. If the surgery obstruction of $\gamma$ is non-zero, then the composite

$$
f: M \stackrel{h}{\rightarrow} X \times S^{k} \stackrel{\text { Proj }}{\longrightarrow} S^{k}
$$

does not fiber. It always fibers stably.

Proof. The composite fibers stably by Proposition 5.8. Suppose there exists a fiber bundle $F \rightarrow M \stackrel{p}{\rightarrow} S^{k}$ homotopic to $f$. We then can lift the homotopy to obtain a homotopy

$$
H: M \times I \rightarrow X \times S^{k} \times I
$$


over $S^{k}$ which restricts to $h$ at $M \times 0$ and which is a fiber homotopy equivalence $F \rightarrow X \times S^{k}$ over 1 . Taking a transverse preimage over the base point of $S^{k}$ yields a degree one normal cobordism

$$
(W ; N, F) \rightarrow\left(X \times S^{k} \times I ; X \times S^{k} \times 0, X \times S^{k} \times 1\right)
$$

whose restriction over 1 is a homotopy equivalence. The restriction over 0 corresponds to the element $\gamma$, which therefore has surgery obstruction 0 , contradicting the assumption.

The following argument how to construct such a simple homotopy equivalence $h$ has been communicated to the author by Tibor Macko. Given $\gamma: X \rightarrow$ $G /$ TOP, by homotopy invariance we can think of $\gamma$ as a normal invariant on $X \times D^{k}$ (without boundary conditions). By the $\pi-\pi$ theorem (here we use the dimension assumptions), this normal invariant is cobordant to a simple homotopy equivalence $Z \rightarrow X \times D^{k}$ which restricts to a simple homotopy equivalence $h^{\prime}: \partial Z \rightarrow X \times S^{k-1}$. Let $M$ be the double of $Z$; then $h^{\prime}$ induces a simple homotopy equivalence $h: M \rightarrow X \times S^{k}$ whose normal invariant over $X \times\{*\}$ is $\gamma$ as desired.

\section{DIFF vs. TOP}

The last example in this section deals with a map that stably fibers in TOP but not in DIFF. It is a slight generalization of an example given by Klein-Williams [KW09].

Proposition 5.18. Let

$$
S^{3} \rightarrow E \stackrel{p}{\rightarrow} S^{3}
$$

be the fibration which corresponds to the generator of $\pi_{3}\left(B G\left(S^{3}\right)\right) \cong \pi_{5}\left(S^{3}\right) \cong$ $\mathbb{Z} / 2$, and let $\lambda: M \rightarrow E$ be any homotopy equivalence from a compact smooth manifold $M$ to $E$. Then $p \lambda$ stably fibers in TOP but not in DIFF.

Proof. By [KW09, Theorem G], $\operatorname{Wall}^{\mathrm{TOP}}(p)=0$ but $\operatorname{Wall}^{\mathrm{DIFF}}(p) \neq 0$. As $E$ is simply-connected, the obstruction group for the fibering obstruction $o(p \lambda)$ is zero.

It would be interesting to have an example of a map that fibers in TOP where the smooth Wall obstruction is zero, but the smooth fibering obstruction is not. This would probably require a deeper analysis of the higher homotopy type of $\mathrm{Wh}^{P L}(F)$ and $\mathrm{Wh}^{\mathrm{DIFF}}(F)$ for a suitable $F$ whose fundamental group has non-vanishing Whitehead group. 


\subsection{A spectral sequence}

The goal of this section is to prove the following spectral sequence result, which will allow, in certain cases, to analyze the Wall obstruction further:

Theorem 5.19. (i) Let $E \rightarrow B$ be a fibration over a $C W$ complex, with fiber $F_{b}$ over $b$. There is a 4 th quadrant spectral sequence

$$
E_{2}^{p, q}=H^{p}\left(B ; \pi_{-q} \mathrm{Wh}\left(F_{b}\right)\right) \Longrightarrow H^{p+q}\left(B ; \mathrm{Wh}\left(F_{b}\right)\right),
$$

where the $E_{2}$-term consists of ordinary cohomology with twisted coefficients in the system of abelian groups $\left\{b \mapsto \pi_{-q} \mathrm{Wh}\left(F_{b}\right)\right\}$.

(ii) If $B$ is $d$-dimensional, $d<\infty$, then the corresponding filtration

$$
\cdots \supset \mathscr{F}^{p, q} \supset \mathscr{F}^{p+1, q-1} \supset \ldots
$$

of $H^{p+q}\left(B ; \mathrm{Wh}\left(F_{b}\right)\right)$ is finite, and the spectral sequence converges in the strongest possible sense, i.e. we have

$$
\begin{aligned}
\mathscr{F}^{0, n} & =H^{n}\left(B ; \mathrm{Wh}\left(F_{b}\right)\right) & & \text { for all } n \\
\mathscr{F}^{d+1, n-d-1} & =0 & & \text { for all } n \\
\mathscr{F}^{p, q} / \mathscr{F}^{p+1, q-1} & \cong E_{\infty}^{p, q} & & \text { for all } p, q
\end{aligned}
$$

(iii) Under the edge homomorphism

$$
H^{0}\left(B ; \mathrm{Wh}\left(F_{b}\right)\right) \rightarrow H^{0}\left(B ; \pi_{0} \mathrm{Wh}\left(F_{b}\right)\right) \subset \coprod_{[b] \in \pi_{0} B} \tilde{K}_{0}\left(\mathbb{Z}\left[\pi_{1} F_{b}\right]\right),
$$

the image of Wall $(p)$ is the finiteness obstruction of the fiber.

(iv) Suppose that all the fibers are homotopy equivalent to finite CW complexes. Let $\gamma: S^{1} \rightarrow B$ be a loop. The naturally defined secondary homomorphism

$$
\operatorname{ker}\left(H^{0}\left(B ; \mathrm{Wh}\left(F_{b}\right)\right) \rightarrow H^{0}\left(B ; \pi_{0} \mathrm{Wh}\left(F_{b}\right)\right)\right) \rightarrow H^{1}\left(B ; \pi_{1} \mathrm{Wh}\left(F_{b}\right)\right),
$$

followed by the restriction map

$$
\gamma^{*}: H^{1}\left(B ; \pi_{1} \mathrm{Wh}\left(F_{b}\right)\right) \rightarrow H^{1}\left(S^{1} ; \pi_{1} \mathrm{Wh}\left(F_{b}\right)\right) \cong \mathrm{Wh}\left(\pi F_{b}\right)_{\pi_{1}\left(S^{1}\right)}
$$

(coinvariants under the $\pi_{1}\left(S^{1}\right)$-action) sends Wall $(p)$ to the element defined by the torsion of the fiber transport along $\gamma$. 
Recall that

$$
\mathbb{H}^{\bullet}\left(B ; \mathrm{Wh}\left(F_{b}\right)=\Gamma\left(\begin{array}{c}
\mathrm{Wh}_{B}(E) \\
\downarrow \\
B
\end{array}\right) \simeq \operatorname{holim}_{\sigma \in \operatorname{simp} B} \operatorname{Wh}\left(E_{\sigma}\right) .\right.
$$

Setting $\mathscr{C}:=\operatorname{simp} B$, the last term may be seen as the $\mathscr{C}$-equivariant cohomology [DL98]

$$
H_{\mathscr{C}}^{\bullet}\left(E \mathscr{C} ; \mathrm{Wh}\left(E_{\sigma}\right)\right)
$$

of $E \mathscr{C}$ with respect to the functor $\sigma \mapsto \mathrm{Wh}\left(E_{\sigma}\right)$ (taking values in $\Omega$-spectra).

For such a $\mathscr{C}$-equivariant cohomology theory $h^{*}$, the authors also provide an Atiyah-Hirzebruch type spectral sequence [DL98, Theorem 4.7]. It converges against $h^{*}(X)$ for a $\mathscr{C}$-CW complex $X$ (with CW-filtration $\left(X^{n}\right)_{n \in \mathbb{N}}$ ); its $E_{1}$-page is given by

$$
E_{1}^{p q}=h^{p+q}\left(X^{p}, X^{p-1}\right)=: C_{h^{*+q}}^{p}(X),
$$

which by definition is the cellular cochain complex of $X$ associated to the cohomology theory $h^{*+q}$. The $E_{1}$-differential is precisely the coboundary operator of this cellular cochain complex, such that

$$
E_{2}^{p q}=H^{p}\left(C_{h^{*+q}}^{*}(X)\right)
$$

is the associated cellular homology.

To prove part (i), we let $h^{*}(X):=H_{\mathscr{C}}^{*}\left(X, \mathrm{Wh}\left(E_{\sigma}\right)\right)$ and $X=E \mathscr{C}$. We need to identify this $E_{2}$ term with $H_{\mathscr{C}}^{p}\left(B ; \pi_{-q} \mathrm{Wh}\left(F_{b}\right)\right)$. Therefore we use the standard model $E \mathscr{C}=\mid \mathscr{C} /$ ? $\mid$ and the fact that the differentials in the cellular cochain complex can be computed using incidence numbers. It turns out that the resulting complex is precisely the simplicial cochain complex of $|\mathscr{C}|=\mid \operatorname{simp} B$. with coefficients in $\pi_{-q} \mathrm{Wh}\left(E_{\sigma}\right)$, so we have

$$
E_{2}^{p q} \cong H^{p}\left(|\operatorname{simp} B .| ; \mathrm{Wh}\left(E_{\sigma}\right)\right) .
$$

Now use the fact that the last vertex map defines a homotopy equivalence

$$
|\mathscr{C}|=|\operatorname{simp} B .| \rightarrow|B .|=B
$$

under which the corresponding twisted coefficient systems agree.

Part (ii) is proven in [DL98]. To prove parts (iii) and (iv) we need to look more closely at the spectral sequence to identify the edge and secondary homomorphisms: 
By definition,

$$
\mathscr{F}^{p q}=\lim _{n} \operatorname{ker}\left(h^{p+q}\left(X^{n}\right) \rightarrow h^{p+q}\left(X^{p-1}\right)\right),
$$

such that a descending filtration on $h^{p+q}(X)$ is given by

$$
\cdots \supseteq \mathscr{F}^{p, q} \supseteq \mathscr{F}^{p+1, q-1} \supseteq \ldots
$$

with

$$
\bigcup_{p} \mathscr{F}^{p, k-p}=\mathscr{F}^{0, k}=\lim _{n} h^{k}\left(X^{n}\right)
$$

and exact sequences

$$
0 \longrightarrow \mathscr{F}^{p+1, q-1} \longrightarrow \mathscr{F}^{p q} \longrightarrow E_{\infty}^{p q}
$$

As the spectral sequence is limited to the right half-plane, we have $E_{1}^{00} \supseteq$ $E_{2}^{00} \supseteq \cdots \supseteq E_{\infty}^{00}$ and $E_{2}^{1,-1} \supseteq E_{3}^{1,-1} \supseteq \cdots \supseteq E_{\infty}^{1,-1}$. Thus we obtain exact sequences

$$
\begin{aligned}
& 0 \longrightarrow \mathscr{F}^{1,-1} \longrightarrow \mathscr{F}^{00} \stackrel{\alpha_{0}}{\longrightarrow} E_{2}^{00}, \\
& 0 \longrightarrow \mathscr{F}^{2,-2} \longrightarrow \mathscr{F}^{1,-1} \stackrel{\alpha_{1}}{\longrightarrow} E_{2}^{1,-1}
\end{aligned}
$$

With this notation the edge homomorphism is given by the composite

$$
h^{0}(X) \rightarrow \lim _{n} h^{0}\left(X^{n}\right)=\mathscr{F}^{00} \stackrel{\alpha_{0}}{\rightarrow} E_{2}^{00}
$$

whereas the secondary homomorphism is given by

$$
\alpha_{1}: \mathscr{F}^{1,-1} \rightarrow E_{2}^{1,-1}
$$

We now show that the maps $\alpha_{i}$ can be described explicitly using the cellular chain complex. With this knowledge and using the identification of the parametrized torsion with the classical one in Proposition 3.8, it is not hard to complete the proof of parts (iii) and (iv).

Lemma 5.20. (i) The restriction map

$$
r_{0}: \mathscr{F}^{00}=\lim _{n} h^{0}\left(X^{n}\right) \rightarrow E_{1}^{00}=h^{0}\left(X^{0}\right)=C_{h^{*}}^{0}(X)
$$

takes values only in cocycles $Z^{0}$ of the cellular cochain complex. 
(ii) The map $\alpha_{0}$ is the composite $\mathscr{F}^{00} \stackrel{r_{0}}{\rightarrow} Z^{0} \rightarrow H_{\mathscr{C}}^{0}\left(X ; \pi_{0} E\right)=E_{2}^{00}$.

(iii) The restriction map $r_{1}: h^{0}\left(X, X^{0}\right) \rightarrow h^{0}\left(X^{1}, X^{0}\right)=C_{h^{*-1}}^{1}(X)$ takes values only in the cocycles $Z^{1}$ of the cochain complex.

(iv) Restriction defines a map $h^{0}\left(X, X^{0}\right) \rightarrow \mathscr{F}^{1,-1}$ such that the following diagram commutes:

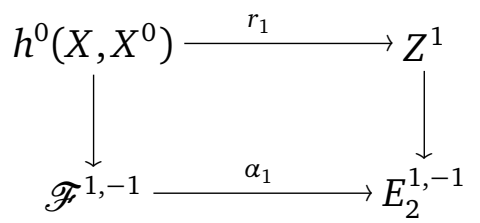

Here the vertical map on the right hand side is the projection of the 1cocycles to 1-cohomology classes of the cochain complex $C_{h^{*-1}}^{*}(X)$.

Proof. Assertions (i) and (iii) are clear. For assertions (ii) and (iv), we need to revisit the construction of the spectral sequence. Define

$$
\begin{aligned}
& Z_{r}^{p q}=\operatorname{im}\left(h^{p+q}\left(X^{p+r-1}, X^{p-1}\right) \rightarrow h^{p+q}\left(X^{p}, X^{p-1}\right)\right), \\
& Z_{\infty}^{p q}=\bigcap_{r} Z_{r}^{p q}, \\
& B_{r}^{p q}=\operatorname{im}\left(h^{p+q-1}\left(X^{p-1}, X^{p-r}\right) \rightarrow h^{p+q}\left(X^{p}, X^{p-1}\right)\right), \\
& B_{\infty}^{p q}=\bigcup_{r} B_{r}^{p q} .
\end{aligned}
$$

Then we have

$$
0=B_{1}^{p q} \subseteq B_{2}^{p q} \subseteq \cdots \subseteq B_{\infty}^{p q} \subseteq Z_{\infty}^{p q} \subseteq \cdots \subseteq Z_{2}^{p q} \subseteq Z_{1}^{p q}=E_{1}^{p q}
$$

and, by construction of the spectral sequence, the following is true:

- $E_{r}^{p q}=Z_{r}^{p q} / B_{r}^{p q}$ and $E_{\infty}^{p q}=Z_{\infty}^{p q} / B_{\infty}^{p q}$.

- Under this identification, the map $\mathscr{F}^{p q} \rightarrow E_{\infty}^{p q}$ is induced by the restriction map $h^{p+q}\left(X^{n}, X^{p-1}\right) \rightarrow h^{p+q}\left(X^{p}, X^{p-1}\right)$.

To see part (ii), observe that, firstly, $Z_{2}^{00}=Z^{0}$ such that the restriction $r_{0}: \mathscr{F}^{00} \rightarrow Z^{0}$ amounts to the composite $\mathscr{F}^{00} \rightarrow Z_{\infty}^{00} \hookrightarrow Z_{2}^{00}$. Secondly, $B_{r}^{00}=0$ for all $r$, thus $Z_{r}^{00}=E_{r}^{00}$, and the inclusion $E_{\infty}^{00} \rightarrow E_{2}^{00}$ is really the inclusion $Z_{\infty}^{00} \rightarrow Z_{2}^{00}$. But the composition $\mathscr{F}^{00} \rightarrow E_{\infty}^{00} \stackrel{\infty}{\hookrightarrow} E_{2}^{00}$ was the definition of the map $\alpha_{0}$. 
For part (iv), we have $\mathscr{F}^{1,-1}=\lim _{n} \operatorname{ker}\left(h^{0}\left(X^{n}\right) \rightarrow h^{0}\left(X^{0}\right)\right)$ and thus the restriction maps $h^{0}\left(X, X_{0}\right) \rightarrow h^{0}\left(X^{n}\right)$ for all $n$ indeed define a map $h^{0}\left(X, X_{0}\right) \rightarrow$ $\mathscr{F}^{1,-1}$. The following diagram with the obvious maps commutes:

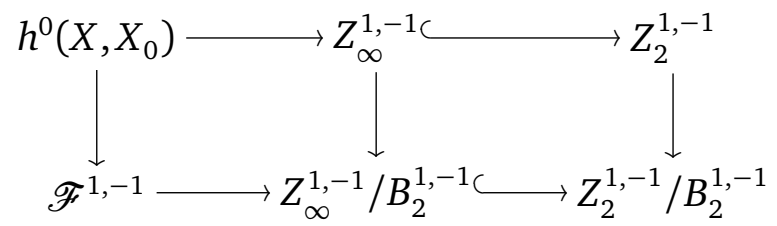

As $B_{2}^{1,-1}=B_{\infty}^{1,-1}$, the middle term in the lower row is $E_{\infty}^{1,-1}$ and the map from $\mathscr{F}^{1,-1}$ to it just the usual one. Moreover, we have $Z_{2}^{1,-1}=Z^{1}$, and the composition of the upper row is the map $r_{1}$. Now we only need to notice that the composition in the lower row is the map $\alpha_{1}$ as defined above.

\subsection{Examples III: Results of Chapman-Ferry}

The obstruction theory developed in this chapter, together with the spectral sequence description of $H^{0}\left(B ; \mathrm{Wh}\left(F_{b}\right)\right)$, allow us to re-obtain results by Chapman-Ferry [CF78] on fibering $Q$-manifolds over compact ANRs. The calculations presented here apply to fibering $Q$-manifolds and to stably fibering finite-dimensional topological manifolds.

Lemma 5.21. Let $p: E \rightarrow B$ be a fibration with homotopy finitely dominated fibers. If the base space is homotopy equivalent to an n-dimensional CW complex, and all the fibers $F_{b}$ are n-connected, then $p$ is fiber homotopy equivalent to $a$ bundle of compact manifolds.

In the case $n=1$, it is enough to suppose $\mathrm{Wh}\left(\pi F_{b}\right)=0$. In the case $n=2$, it is enough to suppose that $F_{b}$ is 1-connected.

Proof. Choose an $n$-dimensional CW complex $B^{\prime}$ and a homotopy equivalence $B^{\prime} \rightarrow B$. Pull the fibration $p$ back to a fibration $p^{\prime}: E \rightarrow B^{\prime}$. If a map $X \rightarrow Y$ is $n$-connected, then the induced map $A(X) \rightarrow A(Y)$ is $n$-connected by [Wal78] and so is $\mathrm{Wh}(X) \rightarrow \mathrm{Wh}(Y)$ by a 5-lemma type argument. As $\mathrm{Wh}(*)$ is contractible, it follows that $\mathrm{Wh}(X)$ is $n$-connected whenever $X$ is $n$-connected. If $X$ is 1-connected, then $\mathrm{Wh}(X)$ is 2-connected (see [Hat78, section 3], with the correction in [Igu84]). Therefore the spectral sequence for $H^{0}\left(B^{\prime} ; \mathrm{Wh}\left(F_{b}\right)\right)$ has vanishing $E_{2}$ page. So Wall $(p)=0$.

Here is our formulation of the results by Chapman-Ferry from [CF78]. Let always $f: M \rightarrow B$ be a map of a compact $Q$-manifold to a compact, connected ANR $B$. 
Proposition 5.22. Suppose B is homotopy equivalent to a finite n-complex. If the homotopy fiber $F$ of $f$ is homotopy finitely dominated and n-connected, then the torsion obstruction $o(f) \in \mathrm{Wh}(\pi M)$ is defined and vanishes if and only if $f$ stably fibers.

If $n=1$, we can replace the assumption " $F$ 1-connected" by "Wh $(\pi F)=0$ ". If $n=2$, we can replace the assumption " $F$ 2-connected" by " $F$ 1-connected".

For example, a two-connected map $f: M \rightarrow S^{2}$ fibers whenever the homotopy fiber of $f$ has the homotopy type of a CW complex and has finitely generated homology.

Proof. By the assumptions and Lemma 5.21, conditions (i) and (ii) are satisfied. Moreover, the map $\alpha: H^{0}\left(B ; \mathrm{Wh}\left(F_{b}\right)\right) \rightarrow \mathrm{Wh}(\pi E)$ is zero since it factors through $\mathrm{Wh}\left(\pi F_{b}\right)=0$.

Proposition 5.23. (i) Suppose that B is homotopy equivalent to a wegde of $n$ copies of $S^{1}(n \geq 1)$, and suppose that the homotopy fiber $F$ of $f$ is connected and homotopy equivalent to a finite complex. Then, Wall $(p)$ is an element of $\bigoplus_{n} \mathrm{Wh}(\pi F)_{\alpha_{n}}$ (coinvariants under the action of the fiber transport along the corresponding copy of $S^{1}$ ).

(ii) The torsion obstruction (whenever defined) is an element in the quotient

$$
\mathrm{Wh}(\pi M) /(n-1) \cdot \operatorname{Wh}(\pi F)^{\pi_{1} B} .
$$

In particular, if $n=1$, the torsion obstruction lives in $\mathrm{Wh}(\pi M)$.

Proof. (i) By the spectral sequence, there is an exact sequence

$$
0 \rightarrow H^{1}\left(B ; \pi_{1} \mathrm{Wh}\left(F_{b}\right)\right) \rightarrow H^{0}\left(B ; \mathrm{Wh}\left(F_{b}\right)\right) \stackrel{e}{\rightarrow} H^{0}\left(B ; \pi_{0} \mathrm{Wh}\left(F_{b}\right)\right) \rightarrow 0,
$$

and the image of Wall $(p)$ under the edge homomorphism $e$ is given by the finiteness obstruction of the fiber, which is zero by assumption. Therefore, Wall $(p)$ lifts to

$$
H^{1}\left(B ; \pi_{1} \mathrm{Wh}\left(F_{b}\right)\right) \cong \bigoplus_{n} H^{1}\left(S^{1} ; \pi_{1} \mathrm{Wh}\left(F_{b}\right)\right) \cong \bigoplus_{n} \mathrm{Wh}\left(\pi F_{b}\right)_{\alpha_{n}} .
$$

(ii) The map $\alpha$ factors as

$$
H^{0}\left(B ; \Omega \mathrm{Wh}\left(F_{b}\right)\right) \rightarrow H^{0}\left(B ; \mathrm{Wh}\left(\pi F_{b}\right)\right) \cong \mathrm{Wh}\left(\pi F_{b}\right)^{\pi_{1} B} \stackrel{\chi_{e}(B) \cdot i_{*}}{\longrightarrow} \mathrm{Wh}(\pi M),
$$

and the Euler characteristic of $B$ is $n-1$. 
Proposition 5.24. Let $f, g: M \rightarrow B$ be two homotopic projections of bundles of compact manifolds. Suppose that $B$ is homotopy equivalent to a wedge of $n$ copies of $S^{1}$. Denote by $F$ the homotopy fiber of $f$, which is homotopy equivalent to the homotopy fiber of $g$. The obstruction group Afor $f$ and $g$ being equivalent fits into the following exact sequence:

$$
0 \rightarrow \bigoplus_{n} \pi_{2} \mathrm{Wh}(F)_{\alpha_{n}} \rightarrow A \rightarrow \mathrm{Wh}(\pi F)^{\pi_{1} B} \stackrel{(n-1) \cdot i_{*}}{\longrightarrow} \mathrm{Wh}(\pi M)
$$

Proof. Recall that $A$ is given by the kernel of

$$
\alpha: H^{0}\left(B ; \Omega \mathrm{Wh}\left(F_{b}\right)\right) \stackrel{\beta}{\rightarrow} H^{0}(B ; \mathrm{Wh}(\pi F)) \cong \mathrm{Wh}(\pi F)^{\pi_{1} B} \stackrel{(n-1) \cdot \dot{i}_{*}}{\rightarrow} \mathrm{Wh}(\pi M) .
$$

By the spectral sequence, we have

$$
0 \rightarrow H^{1}\left(B ; \pi_{2} \mathrm{Wh}\left(F_{b}\right)\right) \rightarrow H^{0}\left(B ; \Omega \mathrm{Wh}\left(F_{b}\right)\right) \stackrel{\beta}{\rightarrow} H^{0}\left(B ; \pi_{1} \mathrm{Wh}\left(F_{b}\right)\right) \rightarrow 0 .
$$

Thus

$$
\operatorname{ker} \beta \cong H^{1}\left(B ; \pi_{2} \mathrm{Wh}\left(F_{b}\right)\right) \cong \bigoplus_{n} \pi_{2} \mathrm{Wh}(F)_{\alpha_{n}} .
$$

Since $\beta$ is surjective, there is a short exact sequence

$$
0 \rightarrow \operatorname{ker} \beta \rightarrow A \rightarrow \operatorname{ker}\left((n-1) \cdot i_{*}\right) \rightarrow 0 \text {. }
$$

The claim follows.

\subsection{Comparison with the obstructions by Farrell-Lück-Steimle}

The content of the author's Diploma thesis [Ste07] was to define obstructions to fibering a manifold over another manifold. See [FLS09] for a published and extended version. The goal of this section is to compare these obstructions.

Given a map $f: M \rightarrow B$ of topological manifolds, factor as usual as $f=$ $p \circ \lambda$, a homotopy equivalence followed by a fibration. Recall that in [FLS09], two obstructions for $f$ to be homotopic to a fiber bundle are defined:

(i) An element $\theta(f) \in H^{1}(B$; $\mathrm{Wh}(\pi M))$ which is defined whenever the homotopy fiber $F$ of $f$ is homotopy finite (an obvious necessary condition). 
It is defined by the rule that whenever $\gamma: S^{1} \rightarrow B$ is a loop in $B$, then under the restriction map

$$
H^{1}(B ; \mathrm{Wh}(\pi M)) \stackrel{r^{*}}{\rightarrow} H^{1}\left(S^{1} ; \mathrm{Wh}(\pi M)\right) \cong \mathrm{Wh}(\pi M)
$$

$\theta(f)$ maps to $i_{*}(\tau)$, where $\tau$ is the Whitehead torsion of the fiber transport on $p$ along $\gamma$ (choosing an arbitrary simple structure on the fiber F).

(ii) If $\theta(f)=0$, there is defined an element

$$
\tau_{\text {fib }}(f) \in \operatorname{coker}\left(\mathrm{Wh}(\pi F) \stackrel{\chi_{e}(B) \cdot i_{*}}{\longrightarrow} \mathrm{Wh}(\pi M)\right)
$$

where $i: F \rightarrow M$ is the inclusion of the homotopy fiber, and $\chi_{e}(B)$ denotes the Euler characteristic. It is defined as follows: Choose a simple structure on the homotopy fiber of $f$ and perform a certain construction (inductively over the cells of $B$ ) to obtain a simple structure on $E$. Then $\tau_{\text {fib }}(f)$ is the image of the Whitehead torsion of $\lambda: M \rightarrow B$, which is well-defined in the quotient.

Theorem 5.25. (i) The image of Wall( $p)$ under the restriction

$$
H^{0}\left(B ; \mathrm{Wh}\left(F_{b}\right)\right) \rightarrow H^{0}\left(\{b\} ; \mathrm{Wh}\left(F_{b}\right)\right) \cong \tilde{K}_{0}\left(\mathbb{Z}\left[\pi F_{b}\right]\right)
$$

is the finiteness obstruction of the fiber.

(ii) Suppose that $F$ is homotopy finite. The image of the Wall obstruction Wall $(p)$ under the secondary homomorphism

$$
\begin{aligned}
\operatorname{ker}\left(H^{0}\left(B ; \mathrm{Wh}\left(F_{b}\right)\right) \rightarrow H^{0}(B\right. & \left.\left.; \pi_{0} \mathrm{Wh}\left(F_{b}\right)\right)\right) \\
& \rightarrow H^{1}\left(B ; \mathrm{Wh}\left(\pi F_{b}\right)\right) \stackrel{i_{*}}{\rightarrow} H^{1}(B ; \mathrm{Wh}(\pi M))
\end{aligned}
$$

is $\theta(f)$.

(iii) Suppose that Wall $(p)=0$. The definition of the map $\alpha$ as a composite

$$
H^{0}\left(B ; \Omega \mathrm{Wh}\left(F_{b}\right)\right) \rightarrow \Omega \mathrm{Wh}\left(F_{b}\right) \stackrel{\chi_{e}(B) \cdot i_{*}}{\longrightarrow} \Omega \mathrm{Wh}(E)
$$

induces a map

$$
\operatorname{coker}\left(\pi_{0}(\alpha)\right) \rightarrow \operatorname{coker}\left(\mathrm{Wh}(\pi F) \stackrel{\chi_{e}(B) \cdot i_{*}}{\longrightarrow} \mathrm{Wh}(\pi M)\right)
$$

under which $o(f)$ maps to $\tau_{\mathrm{fib}}(f)$. In particular, if $\chi_{e}(B)=0$, then

$$
o(f)=\tau_{\text {fib }}(f) \in \mathrm{Wh}(\pi M) .
$$


Proof. (i) and (ii) were proved in Theorem 5.19 .

(iii) If Wall $(p)=0$, then we may assume that $p$ is a bundle of compact topological manifolds, and it follows from [FLS09, Lemmas 3.11 and 3.16] that the simple structure on $E$ is just the canonical simple structure of the topological manifold $E$. Therefore both $o(f)$ and $\tau_{\text {fib }}(f)$ are given by the respective classes of the Whitehead torsion of $\lambda$. 


\section{A Some results on fibrations}

This appendix collects the technical results on fibrations needed to make our classifying machinery work. Again recall that all the spaces are compactly generated Hausdorff.

\section{A.1 The fibered homotopy extension property}

We begin by stating a useful fact.

Lemma A.1 (Fiber homotopy extension property). A map $i: A \rightarrow X$ is a cofibration if and only if the fibered homotopy extension property holds:

Given any (solid) commutative diagram

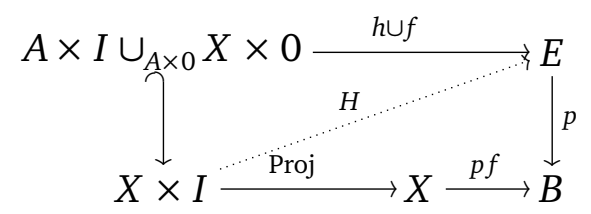

with $p$ a fibration, there exists a (dotted) lift.

In fact, the usual HEP for cofibrations amounts to letting $B$ be a point. On the other hand, if $i$ is a cofibration, the existence of a lift is a standard result.

As a consequence, some well-known results from the theory of cofibrations also hold in the fibered context. Proofs are identical, replacing the homotopy extension property by the fibered one.

Proposition A.2 ([May99, Chapter 6]). Let

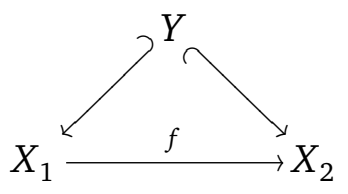

be a diagram of spaces over $B$ (with fiberwise maps), such that the diagonal maps are cofibrations and both $X_{1}$ and $X_{2}$ are fibrations over $B$. If $f$ is a fiber homotopy equivalence, then it is a fiber homotopy equivalence relative to $Y$ (i.e. there is a fiber homotopy inverse and homotopies that fix $Y$ ). 
Notice that any homotopy equivalence between two fibrations is a fiber homotopy equivalence [May99, chapter 7].

Corollary A.3. Suppose that $p: E \rightarrow B$ is a fibration, and that $i: E^{\prime} \hookrightarrow E$ is a cofibration such that $\left.p\right|_{E^{\prime}}: E^{\prime} \rightarrow B$ is also a fibration. Then, $i$ is a homotopy equivalence if and only if $i$ is a fiberwise (strong) deformation retract.

Proposition A.4 ([May99, Chapter 6]). Let

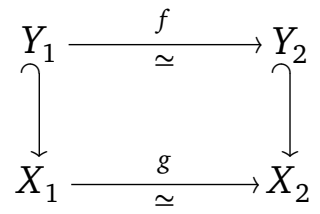

be a diagram of spaces over $B$, with vertical maps cofibrations and $X_{1}$ and $X_{2}$ fibrations over $B$. If $f$ has a fiber homotopy inverse $f^{\prime}$ over $B$ and $g$ is a homotopy equivalence, then $(g, f)$ is a fiber homotopy equivalence of pairs.

More precisely, if $H: Y_{2} \times I \rightarrow Y_{2}$ is a homotopy between id and $f^{\prime} \circ f$, then there exists a fiber homotopy inverse $g^{\prime}$ of $g$ that extends $f^{\prime}$ such that $H$ extends to a homotopy $K: X_{2} \times I \rightarrow X_{2}$ between id and $g^{\prime} \circ g$.

\section{A.2 Associated fibration and connections}

For $f: X \rightarrow B$, denote by $\mathscr{E}(X) \rightarrow B$ the functorially associated fibration, with $\mathscr{E}(X)$ being the following pull-back:

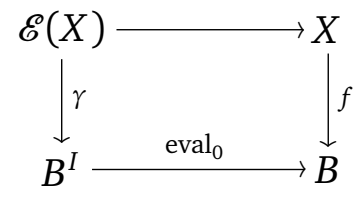

Lemma A.5. If $B$ is metrizable, and $p: E \rightarrow B$ is a fibration, then the inclusion $E \subset \mathscr{E}(E)$ is a fiberwise (strong) deformation retract.

Proof. The inclusion $B \rightarrow B^{I}$ of constant functions is a (strong) deformation retract; so it is a cofibration if there is a map $\varphi: B^{I} \rightarrow I$ such that $B=\varphi^{-1}(0)$. If $d$ is a metric on $B$, then

$$
\varphi(a)=\sup _{x \in I}(d(a(0), a(x)))
$$

is clearly such a map.

Now, since the inclusion $B \rightarrow B^{I}$ is a section of the fibration eval ${ }_{0}$, it induces a section of the fibration $\mathscr{E}(X) \rightarrow X$, which then is also a cofibration. 
Corollary A.6 (Hurewicz). Any fibration over a metrizable space is regular, i.e. homotopy lifting problems can be solved in such a way that constant paths are lifted to constant paths.

Proof. In fact whenever a map $p: X \rightarrow B$ is a retract of its associated fibration $\mathscr{E}(X) \rightarrow B$, then it is a regular fibration. This is because any homotopy lifting problem

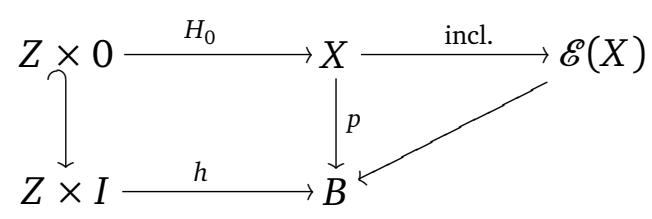

has a regular solution in $\mathscr{E}(X)$ given by

$$
H: Z \times I \rightarrow \mathscr{E}(X), \quad(z, t) \mapsto\left(H_{0}(z), \gamma\right) \quad \text { with } \quad \gamma(s)=h(z, s \cdot t)
$$

Now use the retraction to obtain a regular solution in $X$.

For a map $p: E \rightarrow B$, a connection is a lift in the following diagram

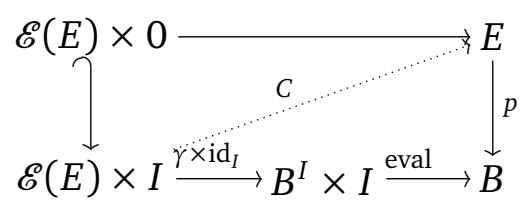

Clearly, if $p$ is a fibration, then a connection always exists. Conversely, the existence of a connection implies that $p$ is a fibration. Indeed, to give a homotopy lifting problem

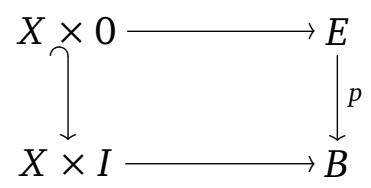

is the same thing as to give a map $\alpha: X \rightarrow \mathscr{E}(E)$, and the composite $C \circ(\alpha \times$ $\left.\mathrm{id}_{I}\right): X \times I \rightarrow E$ is then a solution of the homotopy lifting problem.

\section{A.3 Fiberwise glueing}

There are two possible ways of glueing fibrations along cofibrations: Firstly, glueing the fibers and keeping the base space fixed, and secondly, glueing fibrations with same fibers over different base spaces. We begin with the first case.

Here are two lemmas that we will use without further remarks. 
Lemma A.7 ([Lüc89, Lemma 1.26]). Let

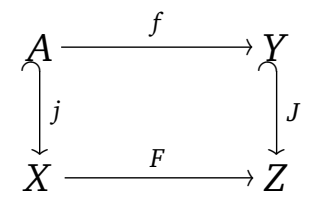

be a push-out with $j$ a cofibration, and let $p: E \rightarrow Z$ be a fibration. Then the induced square

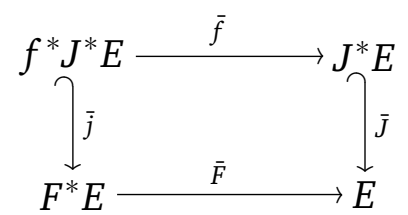

is a push-out with $\bar{j}$ a cofibration.

Lemma A.8. Let $E \rightarrow B$ be a map, and let

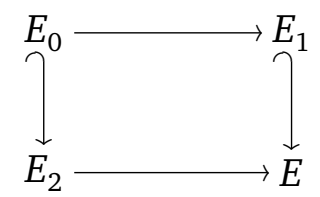

be a push-out. Then, for $f: A \rightarrow B$, the diagram

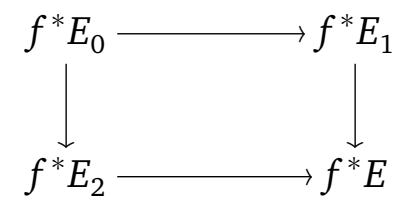

is a push-out, too.

We will see down below that, in the case of fibrations, the vertical maps are actually cofibrations. All we need to know for the proof is that they are topological inclusions.

Proof. Consider the push-out $P:=f^{*} E_{1} \cup_{f^{*} E_{0}} f^{*} E_{2}$ in the topological category. The map from $P$ to $f^{*} E$ is a continuous bijection. To show that it is a homeomorphism, it is enough to show that $P$ carries the subspace topology of $Q:=E_{1} \times A \cup_{E_{0} \times A} E_{2} \times A$ (indeed the natural map $Q \rightarrow E \times A$ is a homeomorphism). Therefore we are going to show that the injective continuous map $P \rightarrow Q$ is closed. 
It is easy to see that the inclusion of $f^{*} E_{i}$ into $E_{i} \times A$ is a closed embedding for $i=0,1,2$. Now a subset $Z \subset P$ is closed if and only if it is the image of $\bar{Z} \subset f^{*} E_{1} \bigsqcup f^{*} E_{2}$ which is both closed and saturated, i.e. if for $x \in f^{*} E_{0}$, we have $x \in \bar{Z}$ if and only if $f(x) \in \bar{Z}$.

Now $\bar{Z}$ considered as a subset of $f^{*} E_{1} \bigsqcup f^{*} E_{2}$ is closed and saturated if and only if it is closed and saturated as a subset of $E_{1} \times A \coprod E_{2} \times A$. In this case, the image of $Z$ in $Q$ is closed again.

A particularly simple case of glueing arises when all the maps involved are cofibrations.

Lemma A.9. Let

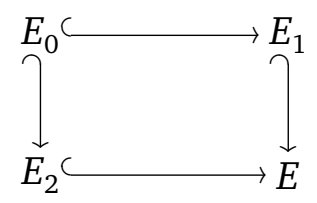

be a push-out square of spaces, with all maps cofibrations, and let $p: E \rightarrow B$ be a map. If $\left.p\right|_{E_{i}}: E_{i} \rightarrow B$ are fibrations for $i=0,1,2$, then so is $p$.

Proof. Since $E_{0} \rightarrow E_{i}$ is a cofibration $(i=1,2)$, so is $\mathscr{E}\left(E_{0}\right) \rightarrow \mathscr{E}\left(E_{i}\right)$. So we can find connections $C_{i}$ for $E_{i}$ that are compatible with a given connection $C_{0}$ for $E_{0}$. We thus obtain a connection

$$
\mathscr{E}(E)=\mathscr{E}\left(E_{1}\right) \cup_{\mathscr{E}\left(E_{0}\right)} \mathscr{E}\left(E_{2}\right) \rightarrow E=E_{1} \cup_{E_{0}} E_{2} .
$$

Corollary A.10. Let $f: E^{\prime} \rightarrow E$ be a fiberwise map between fibrations over $B$. If $f$ is a cofibration, then

(i) the canonical map from the mapping cylinder to $B$ is a fibration, and

(ii) for any map $g: A \rightarrow B$, the induced map $g^{*} f: g^{*} E^{\prime} \rightarrow g^{*} E$ is a cofibration.

So, if a fiberwise map between fibratons is a cofibration on the level of total spaces, then it is also a cofibration on each fiber. The converse statement does not hold, as can be easily seen using Tulley's construction (described below).

Proof. (i) follows directly from Lemma A.9.

(ii) The map $f$ being a cofibration is equivalent to saying the the canonical map $\operatorname{Cyl}(f) \rightarrow E \times I$ is a cofibration and a homotopy equivalence. So, as $\operatorname{Cyl}(f)$ is a fibration over $B$, Corollary A.3 implies that the inclusion is a fiberwise deformation retract. Therefore its restriction, namely the inclusion $\operatorname{Cyl}\left(g^{*} f\right) \rightarrow g^{*} E \times I$, is still a retract. 
If we relax the cofibration condition somewhat, we have to put stronger hypotheses on the base space:

Theorem A.11 ([Arn73, Thm. 2.5]). Lemma A.9 still holds if we only assume that the vertical maps in Diagram (1) are cofibrations, provided that $B$ is metrizable ULC.

In particular, the mapping cylinder of a fiberwise map between fibrations over such spaces $B$ is again a fibration over $B$.

\section{A.4 Glueing over different base spaces}

Proposition A.12 ([ Arn72, Thm. 4.2]). Let $X$ be metrizable, let

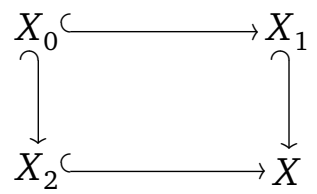

be a push-out square with all maps cofibrations, and $X_{i} U L C(i=1,2)$. (This condition is satisfied if $X_{i}$ are $C W$ spaces.) Then if $B$ is another metrizable space, and $p: E \rightarrow B \times X$ is a map such that the restriction of $p$ to $p^{-1}\left(B \times X_{i}\right) \rightarrow B \times X_{i}$ is a fibration ( $i=1,2)$, then $p$ is a fibration.

Proof of Proposition A.12 We are going to show that for each $x \in X_{0}$ there is a neighborhood $U$ of $x$ in $X$ such that $p$, restricted over $B \times U$ is a fibration.

The proof uses the observation that any fiberwise retract of a fibration is a fibration. In our case, write $p=\left(p_{1}, p_{2}\right)$, and let, for some $U \subset X, q=p_{1} \times$ $\operatorname{id}_{U}:\left.E\right|_{B \times U} \times U \rightarrow B \times U$. The map $q$ is a fibration, and there is a commutative diagram

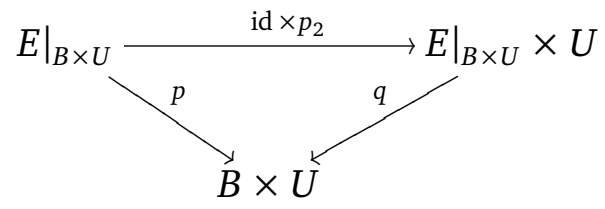

So it is enough to construct a fiberwise map (often called "slicing function") $\varphi:\left.E\right|_{B \times U} \times\left. U \rightarrow E\right|_{B \times U}$ such that $\varphi \circ\left(\mathrm{id} \times p_{2}\right)=\mathrm{id}$.

The hypotheses on the base spaces imply that there is a neighborhood $U$ of $x$ and a homotopy

$$
\sigma: U \times U \times I \rightarrow X
$$


between the projection onto the first and the projection onto the second factor, such that the homotopy is stationary on the diagonal and, for each $(x, y) \in$ $U \times U$, the corresponding path $\alpha$ from $x$ to $y$ has the following additional property:

For each $i, j$, if $\alpha(0) \in X_{i}$, then $\alpha\left(\left[0, \frac{1}{2}\right]\right) \subset X_{i}$, and if $\alpha(1) \in X_{j}$, then $\alpha\left(\left[\frac{1}{2}, 1\right]\right) \subset X_{j}$.

Consider now the following homotopy lifting problem:

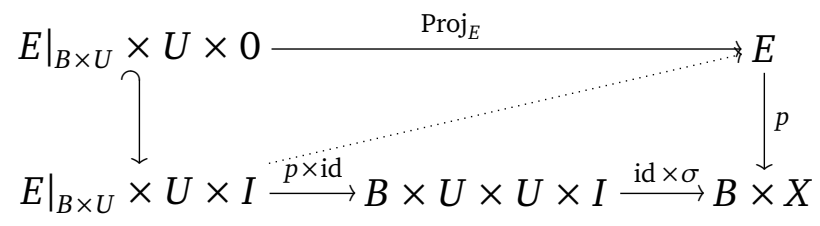

This particular problem can be solved at least on $\left.E\right|_{B \times U} \times U \times\left[0, \frac{1}{2}\right]$ (first solve it on $\left.E\right|_{B_{0} \times U} \times U \times\left[0, \frac{1}{2}\right]$ and then extend using the fibered homotopy extension property). Then we can solve the problem similarly on $\left.E\right|_{B \times U} \times U \times\left[\frac{1}{2}, 1\right]$, thus obtaining a solution of the whole problem.

As both $B$ and $X$ are metrizable, there is a regular solution by Corollary A.6. Such a solution of our problem, evaluated at 1 , is the map $\varphi$ we need.

Next we deal with the question whether this glueing procedure preserves the cofibration condition. Here is a preparatory lemma.

Lemma A.13. Let $i: A \hookrightarrow B$ be a cofibration, $p: X \rightarrow B$ a fibration, $j: Y \hookrightarrow X$ a cofibration such that $\left.p\right|_{Y}: Y \rightarrow B$ is a fibration. Then the inclusion

$$
\left.X\right|_{A} \cup Y \hookrightarrow X
$$

is a cofibration.

Proof. This is an adapted version of the proof of Strøm [Str68, Theorem 13]. By Strøms version of the NDR property (see [Whi78, I.5.14]), the inclusion of $A$ into $B$ being a cofibration is equivalent to the existence of $H: B \times I \rightarrow B$ and $\varphi: B \rightarrow I$ such that

(i) $A \subset \varphi^{-1}(0)$,

(ii) $H$ is stationary on $A$,

(iii) $H_{0}=\mathrm{id}_{B}$,

(iv) $H(b, t) \in A$ whenever $\varphi(b)<t$. 
In fact, any retraction $r: B \times I \rightarrow A \times I \cup B \times 0$ gives rise to

$$
H(x, t):=\operatorname{Proj}_{B} r(x, t) \text { and } \varphi(x):=\sup _{t \in I}\left(t-\operatorname{Proj}_{I} r(x, t)\right) .
$$

As $Y \hookrightarrow X$ is also a cofibration, let $D: X \times I \rightarrow X, \psi: X \rightarrow I$ such that the analogues of conditions (i) to (iv) hold. More precisely, as $Y \times I \cup X \times 0$ is a fiberwise retract of $Y \times I$, we can even assume that $D$ is also a fiber homotopy over $B$.

Let

$$
\begin{array}{rlrl}
\eta & : X \rightarrow I, & \eta(x) & =\min (\psi(x), \varphi p(x)), \\
G: X \times I \rightarrow X, & G(x, t) & =\bar{H}[D(x, \min (t, \varphi p(d))), \min (t, \eta(x))],
\end{array}
$$

where $\bar{H}: X \times I \rightarrow X$ is a lift of $H$ such that $\bar{H}\left(X^{\prime} \times I\right) \subset X^{\prime}$ and $\bar{H}_{0}=\mathrm{id}_{X}$.

Then,

(i) $\left.X\right|_{A} \cup Y \subset \eta^{-1}(0)$,

(ii) $G$ is stationary on $\left.X\right|_{A} \cup Y$,

(iii) $G_{0}=\mathrm{id}_{X}$,

(iv) if $\eta(x)<t$, then either

(a) $\psi(x)<\varphi p(x)$, in which case $\min (t, \varphi p(x))>\psi(x)$, so

$$
D(x, \min (t, \varphi p(x))) \in Y
$$

and

$$
G(x, t)=\bar{H}[D(x, \min (t, \varphi p(x))), \min (t, \eta(x))] \in Y,
$$

(b) or $\varphi p(x) \leq \psi(x)$ and $\varphi p(x)<1$, in which case $\min (t, \eta(x))=$ $\varphi p(x)$, so

$$
\begin{aligned}
p G(x, t) & =p \bar{H}[D(x, \min (t, \varphi p(x))), \varphi p(x)] \\
& =H[p D(x, \min (t, \varphi p(x))), \varphi p(x)] \\
& =H[p D(x, 0), \varphi p(x)] \\
& =H[p(x), \varphi p(x)] \in A .
\end{aligned}
$$

Proposition A.14. Let $p: E \rightarrow B$ be a fibration, $E^{\prime} \subset E$ a subspace such that $\left.p\right|_{E^{\prime}}: E^{\prime} \rightarrow B$ is a fibration. Suppose that $B$ is a push-out

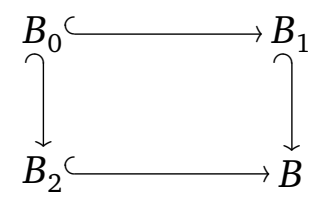


with all maps cofibrations. Let $E_{i}:=\left.E\right|_{B_{i}}$ and $E_{i}^{\prime}:=\left.E^{\prime}\right|_{B_{i}}$. If the inclusions $j_{i}: E_{i}^{\prime} \rightarrow E_{i}$ are cofibrations for $i=0,1,2$, then so is the inclusion $j: E^{\prime} \rightarrow E$.

This proposition follows at once from Lemma A.13 together with the following Lemma whose proof is an exercise using the definitions.

Lemma A.15. Let

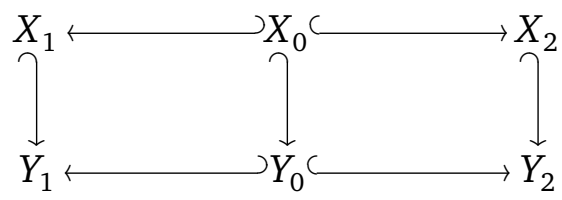

be a diagram of spaces with all maps cofibrations. If the induced maps

$$
X_{1} \cup_{X_{0}} Y_{0} \rightarrow Y_{1} \text { and } X_{2} \cup_{X_{0}} Y_{0} \rightarrow Y_{2}
$$

are cofibrations, then so is

$$
X_{1} \cup_{X_{0}} X_{2} \rightarrow Y_{1} \cup_{Y_{0}} Y_{2}
$$

\section{A.5 Tulley's construction}

Proposition A.16 ([Tu165]). Let $p: E \rightarrow B$ be a fibration over a metrizable space $B$, and let $i: E^{\prime} \rightarrow E$ a cofibration and fiber homotopy equivalence. Denote

$$
T(i):=E^{\prime} \times\{0\} \cup E \times(0,1] \subset E \times I .
$$

Then $\left.p\right|_{E^{\prime}}: E^{\prime} \rightarrow B$ is a fibration, and so is the obvious map $q: T(i) \rightarrow B \times I$ induced by $p$ and $i$.

In fact, it is clear that the restriction of $p$ to $E^{\prime}$ is a fibration if $i$ is a fiberwise retract. Notice that the condition on $i$ implies that $i$ is a fiberwise strong deformation retract by Corollary A.3.

Finally, as Tulley's proof is somewhat involved, we give here a simpler proof.

Proof of Proposition A.16. Let $K: E \times I \rightarrow E$ be a fiber homotopy between $\mathrm{id}_{E}$ and a retraction onto $E^{\prime}$. Let the left square in the following diagram be a homotopy lifting problem that we wish to solve. (Here $J=I=[0,1]$.)

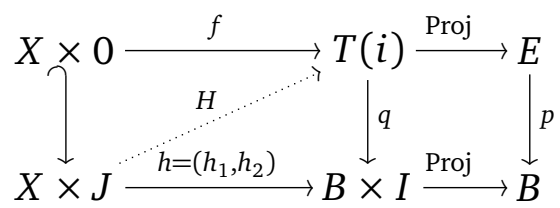


Using the outer square and the fact that $p$ is a fibration, we obtain a lift $L: X \times J \rightarrow E$ of $h_{1}$ that extends the projection of $f$. Now, let $H(x, t):=$ $\left(H_{1}(x, t), h_{2}(x, t)\right) \in E \times I$ with

$$
H_{1}(x, t)= \begin{cases}K\left(L(x, t), \min \left(1, \frac{t}{h_{2}(x, t)}\right)\right), & \text { if } t>0 \\ f(x) & \text { if } t=0\end{cases}
$$

(letting $\min \left(1, \frac{t}{0}\right)=1$ ).

We have to check continuity in $t=0$. So let $\left(x_{\alpha}, t_{\alpha}\right)$ be a net converging to $(x, 0)$. The only nontrivial case is when $h_{2}\left(x_{\alpha}, t_{\alpha}\right)$ tends to 0 , too. In this case $L\left(x_{\alpha}, t_{\alpha}\right)$ converges to $f(x)$ which lies in $E^{\prime}$. But if $e_{\alpha}$ converges to some element $e \in E^{\prime}$, then $K\left(e_{\alpha}, \tau_{\alpha}\right)$ converges to $e$ for any net $\left(\tau_{\alpha}\right)$ on $I$, as $K$ is stationary on $E^{\prime}$ and the unit interval is compact.

Now, whenever $h_{2}(x, t)=0$, we have $H_{1}(x, t) \in E^{\prime}$, thus $H$ really defines a map to $T(i)$. Moreover $p \circ H_{1}(x, t)=h_{1}(x, t)$, as $K$ is a fiber homotopy and $L$ is a lift of $h_{1}$, so $q \circ H=h$.

Lemma A.17. Suppose that we have a square of fibrations

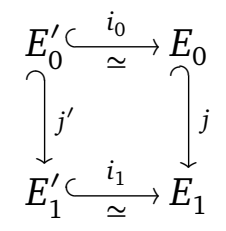

over $B$, with all maps cofibrations and the horizontal maps fiber homotopy equivalences. If the canonical map $E_{1}^{\prime} \cup_{E_{0}^{\prime}} E_{0} \rightarrow E_{1}$ is a cofibration, then so is the inclusion $T\left(i_{0}\right) \rightarrow T\left(i_{1}\right)$.

Proof. Recall that the cofibration condition implies that there are retractions $r: E_{1} \times I \rightarrow$ Cyl $j$ and $r^{\prime}: E_{1}^{\prime} \times I \rightarrow$ Cyl $j^{\prime}$. The additional condition is easily seen to guarantee that $r$ can be modified in such a way that $r$ and $r^{\prime}$ are compatible, i.e. $\left.r\right|_{E_{1}^{\prime}}=r^{\prime}: E_{1}^{\prime} \rightarrow$ Cyl $j$.

Now recall from the proof of Lemma A.13 how to get a NDR pair structure $(\varphi, H)$ on $\left(E_{1}, E_{0}\right)$ from the retraction $r$.

Since $r$ and $r^{\prime}$ are compatible, this implies that $\left(\varphi, H^{\prime}\right)$ and the corresponding maps $\left(\varphi^{\prime}, H^{\prime}\right)$ for the NDR pair $\left(E_{1}^{\prime}, E_{0}^{\prime}\right)$ are compatible. Hence we can define $\bar{\varphi}: T\left(i_{1}\right) \rightarrow I$ by applying either $\varphi$ or $\varphi^{\prime}$ to the first coordinate of $(x, t) \in T\left(i_{1}\right) \subset E_{0} \times I$. Define $\bar{H}: T\left(i_{1}\right) \times I \rightarrow T\left(i_{1}\right)$ in a similar way. The maps $\bar{\varphi}$ and $\bar{H}$ satisfy the properties required for $\left(T\left(i_{1}\right), T\left(i_{0}\right)\right)$ to be an NDR pair. 


\section{A.6 Fill-in for fibrations}

The goal of this sections is to show that fill-ins exist for fibrations and for fibrations with fiberwise splitting (as considered in section 3). We will see that Tulley's construction, together with Theorem A.11, will produce fill-ins. To make sure that we have enough space to deal with homotopies, we redefine now $T(i):=E^{\prime} \times\left[0, \frac{1}{2}\right] \cup E \times\left(\frac{1}{2}, 1\right]$. It can be understood as alueing of the original construction with the trivial fibration $E^{\prime} \times I$. So the essential properties remain unchanged.

Proposition A.18. The functor $\mathbf{F i b}(B ; F)$ from section 2 satisfies the Fill-in Condition if $B$ is metrizable ULC.

For the reader's convenience, and to establish notation, we recall the Fill-in property for our special case:

For any three fibrations $E^{\prime}, E^{\prime \prime}, E$ over $B \times \Delta^{k}$ and any two fiber homotopy equivalences $\varphi^{\prime}: E^{\prime} \rightarrow E$ and $\varphi^{\prime \prime}: E^{\prime \prime} \rightarrow E$, the following holds: There exists a fibration $\bar{E}$ over $\Delta^{k} \times I$ and a fiber homotopy equivalence $\Phi: \bar{E} \rightarrow E \times I$ which restricts to $\varphi^{\prime}$ over 0 and to $\varphi^{\prime \prime}$ over 1 .

Moreover, given two more fibrations $F^{\prime}, F^{\prime \prime}$ over $\Delta^{k}$ and fiber homotopy equivalences $\psi^{\prime}: F^{\prime} \rightarrow E$ and $\psi^{\prime \prime}: F^{\prime \prime} \rightarrow E$, which agree with $\left(F^{\prime}, F^{\prime \prime}, \varphi^{\prime}, \varphi^{\prime \prime}\right)$ when restricted to a collection of faces of $\Delta^{k}$, there are extensions $(\bar{E}, \Phi)$ and $(\bar{F}, \Psi)$ of $\left(E^{\prime}, E^{\prime \prime}, \varphi^{\prime}, \varphi^{\prime \prime}\right)$ and $\left(F^{\prime}, F^{\prime \prime}, \psi^{\prime}, \psi^{\prime \prime}\right)$ that agree when restricted to the same collection $\times I$.

Proof of Proposition A.18. Let us first consider a special case: Suppose that the $\operatorname{map}\left(\varphi^{\prime \prime}\right)^{-1} \circ \varphi^{\prime}: E^{\prime} \rightarrow E^{\prime \prime}$ is fiber homotopic to a map $\alpha$ which is a cofibration and homotopy equivalence. In this case the canonical map $T(\alpha) \rightarrow B$ is a fibration by Proposition A.16, and it is easy to construct a map $\Phi: T(\alpha) \rightarrow E \times I$ as requested, using a fiber homotopy $\varphi^{\prime \prime} \circ \alpha \simeq \varphi^{\prime}$.

In the general case, the mapping cylinder $\operatorname{Cyl}(\alpha)$ is a fibration over $B$ by Theorem A.11. It comes with a canonical map $\hat{\varphi}: \operatorname{Cyl}(\alpha) \rightarrow E^{\prime \prime} \rightarrow E$. In this case both inclusions of $E^{\prime}$ and $E^{\prime \prime}$ into the mapping cylinder are cofibrations and homotopy equivalences. So, by the previous case, fill-ins exist between $\left(E^{\prime}, \varphi^{\prime}\right)$ and $(\operatorname{Cyl}(\alpha), \hat{\varphi})$, and between $(\operatorname{Cyl}(\alpha), \hat{\varphi})$ and $\left(E^{\prime \prime}, \varphi^{\prime \prime}\right)$. Glueing these (using the Amalgamation property), one now obtains a fill-in between $\left(E^{\prime}, \varphi^{\prime}\right)$ and $\left(E^{\prime \prime}, \varphi^{\prime \prime}\right)$.

Proposition A.4 guarantees that this can be chosen to be compatible over smaller simplices. 
The interested reader may want to compare this proof with the intricate argument from [HTW90, Lemma 17.3]. - Now we proceed to show:

Proposition A.19. The functor $\mathbf{F i b}\left(B ;\left(F_{i}\right)\right)$ from section 3 satisfies the Fill-in Condition if $B$ is metrizable ULC.

Recall that the corresponding objects are four fibrations $p_{i}: E_{i} \rightarrow B \times \Delta^{n}$ for $i=0, \ldots, 3$, which are objects of $\operatorname{Fib}\left(B ; F_{i}\right)$ respectively, together with fiberwise inclusions $j_{i}: E_{i} \rightarrow E_{3}$ for $i=0,1,2$ which are cofibrations.

The situation is thus the following: We are given a fibration $E_{3}^{\prime}$ over $B \times \Delta^{k}$, which comes equipped with three sub-fibrations $E_{0}^{\prime}, E_{1}^{\prime}, E_{2}^{\prime}$ such that $E_{3}^{\prime}$ is the push-out of $E_{1}^{\prime}$ and $E_{2}^{\prime}$ over $E_{0}^{\prime}$, and all the inclusions are cofibrations. We are also given two fibrations $E_{3}^{\prime \prime}$ and $E_{3}$ with a similar splitting, and we are given two fiber homotopy equivalences $\varphi_{3}^{\prime}: E_{3}^{\prime} \rightarrow E_{3}$ and $\varphi_{3}^{\prime \prime}: E_{3}^{\prime \prime} \rightarrow E$ which respect the splittings.

Given this, we have to produce a fibration $\bar{E}_{3}$ over $\Delta^{k} \times I$ with similar splitting and a fiber homotopy equivalence $\Phi: \bar{E}_{3} \rightarrow E_{3} \times I$ which respects the splittings and restricts to $\varphi^{\prime}$ over 0 and to $\varphi^{\prime \prime}$ over 1 .

Proof of Proposition A.19 Notice that a given fill-in problem determines, for $i=0, \ldots, 3$ a fill-in problem for $\operatorname{Fib}\left(B ; F_{i}\right)$ which we are going to call the $i$ problem. Obviously Proposition A.18 produces a solution for each $i$-problem separately. Notice however that the construction in the proof even provides a fill-in for the 0-problem which is a subspace of both a fill-in for the 1- and a fillin for the 2-problem. (Just choose $\alpha_{3} \simeq\left(\varphi_{3}^{\prime \prime}\right)^{-1} \circ \varphi_{3}^{\prime}$ respecting the splittings and follow the mapping cylinder construction.) We only have to show that both inclusions of the 0 -fill-in into the 1-fill-in and the 2-fill-in are cofibrations.

To do that, it is enough, by Lemma A.17, to verify that the inclusions

$$
E_{i}^{\prime} \cup_{E_{0}^{\prime}} \operatorname{Cyl} \alpha_{0} \rightarrow \operatorname{Cyl} \alpha_{i} \quad \text { and } \quad E_{i}^{\prime \prime} \cup_{E_{0}^{\prime \prime}} \operatorname{Cyl} \alpha_{0} \rightarrow \operatorname{Cyl} \alpha_{i}, \quad i=1,2
$$

are cofibrations. In fact, the inclusion

$$
E_{i}^{\prime} \cup_{E_{0}^{\prime}} \operatorname{Cyl} \alpha_{0} \rightarrow E_{i}^{\prime} \cup_{E_{0}^{\prime}} \operatorname{Cyl} \alpha_{0} \cup_{E_{0}^{\prime \prime}} E_{i}^{\prime \prime}
$$

is a cofibration since it is obtained by glueing $E_{1}^{\prime \prime}$ along the cofibration $E_{0}^{\prime \prime} \rightarrow$ $E_{1}^{\prime \prime}$. Similarly,

$$
\begin{aligned}
\operatorname{Cyl} \alpha_{0} \cup_{E_{0}^{\prime \prime}} E_{i}^{\prime \prime} & \rightarrow E_{i}^{\prime} \cup_{E_{0}^{\prime}} \operatorname{Cyl} \alpha_{0} \cup_{E_{0}^{\prime \prime}} E_{i}^{\prime \prime} \text { and } \\
E_{i}^{\prime} \cup_{E_{0}^{\prime}} \operatorname{Cyl} \alpha_{0} \cup_{E_{0}^{\prime \prime}} E_{i}^{\prime \prime} & \rightarrow \operatorname{Cyl} \alpha_{i}
\end{aligned}
$$


are cofibrations.

The compatibility result over smaller simplices again holds by Proposition A.4. 


\section{Bibliography}

[And74] Douglas R. Anderson. The Whitehead torsion of a fiber-homotopy equivalence. Michigan Math. J., 21:171-180, 1974.

[Arn72] E. Arnold, James Jr. Local to global theorems in the theory of Hurewicz fibrations. Trans. Amer. Math. Soc., 164:179-188, 1972.

[Arn73] James E. Arnold, Jr. Attaching Hurewicz fibrations with fiber preserving maps. Pacific J. Math., 46:325-335, 1973.

[BD07] Bernard Badzioch and Wojciech Dorabiała. Additivity for the parametrized topological Euler characteristic and Reidemeister torsion. K-Theory, 38(1):1-22, 2007.

[BDW09] Bernard Badzioch, Wojciech Dorabiała, and Bruce Williams. Smooth parametrized torsion: a manifold approach. Adv. Math., 221(2):660-680, 2009.

[BL66] W. Browder and J. Levine. Fibering manifolds over a circle. Comment. Math. Helv., 40:153-160, 1966.

[Bru71] Gregory W. Brumfiel. Homotopy equivalences of almost smooth manifolds. In Algebraic topology (Proc. Sympos. Pure Math., Vol. XXII, Univ. Wisconsin, Madison, Wis., 1970), pages 73-79. Amer. Math. Soc., Providence R.I., 1971.

[Cas67] A. J. Casson. Fibrations over spheres. Topology, 6:489-499, 1967.

[CF78] T. A. Chapman and Steve Ferry. Fibering Hilbert cube manifolds over ANRs. Compositio Math., 36(1):7-35, 1978.

[Cha76] T. A. Chapman. Lectures on Hilbert cube manifolds. American Mathematical Society, Providence, R. I., 1976. Expository lectures from the CBMS Regional Conference held at Guilford College, October 11-15, 1975, Regional Conference Series in Mathematics, No. 28.

[Coh73] Marshall M. Cohen. A course in simple-homotopy theory. Springer-Verlag, New York, 1973. Graduate Texts in Mathematics, Vol. 10.

[DL98] James F. Davis and Wolfgang Lück. Spaces over a category and assembly maps in isomorphism conjectures in $K$ - and $L$-theory. $K$-Theory, 15(3):201-252, 1998. 
[Dor02] Wojciech Dorabiała. The double coset theorem formula for algebraic $K$ theory of spaces. K-Theory, 25(3):251-276, 2002.

[DWW03] W. Dwyer, M. Weiss, and B. Williams. A parametrized index theorem for the algebraic K-theory Euler class. Acta Math., 190(1):1-104, 2003.

[EW42] Samuel Eilenberg and R. L. Wilder. Uniform local connectedness and contractibility. Amer. J. Math., 64:613-622, 1942.

[Far72] F. T. Farrell. The obstruction to fibering a manifold over a circle. Indiana Univ. Math. J., 21:315-346, 1971/1972.

[Fer77] Steve Ferry. The homeomorphism group of a compact Hilbert cube manifold is an ANR. Ann. Math. (2), 106(1):101-119, 1977.

[FLS09] F. T. Farrell, Wolfgang Lück, and Wolfgang Steimle. Obstructions to fibering a manifold. Geom. Dedicata, 2009. Online.

[Han51] Olof Hanner. Some theorems on absolute neighborhood retracts. Ark. Mat., 1:389-408, 1951.

[Hat78] A. E. Hatcher. Concordance spaces, higher simple-homotopy theory, and applications. In Algebraic and geometric topology (Proc. Sympos. Pure Math., Stanford Univ., Stanford, Calif., 1976), Part 1, pages 3-21. Amer. Math. Soc., Providence, R.I., 1978.

[Hir78] F. Hirzebruch. Topological mathods in algebraic geometry, volume 131. Springer, 1978.

[Hoe09] S. Hoehn. The moduli space of compact fiber bundle structures on a fibration. PhD thesis, University of Notre Dame, 2009.

[HTW90] C. B. Hughes, L. R. Taylor, and E. B. Williams. Bundle theories for topological manifolds. Trans. Amer. Math. Soc., 319(1):1-65, 1990.

[Igu84] Kiyoshi Igusa. What happens to Hatcher and Wagoner's formulas for $\pi_{0} C(M)$ when the first Postnikov invariant of $M$ is nontrivial? In $A l-$ gebraic K-theory, number theory, geometry and analysis (Bielefeld, 1982), volume 1046 of Lecture Notes in Math., pages 104-172. Springer, Berlin, 1984.

[Igu88] Kiyoshi Igusa. The stability theorem for smooth pseudoisotopies. $K$ Theory, 2(1-2):vi+355, 1988.

[Kis64] J. M. Kister. Microbundles are fibre bundles. Ann. of Math. (2), 80:190199, 1964. 
[KS77] Robion C. Kirby and Laurence C. Siebenmann. Foundational essays on topological manifolds, smoothings, and triangulations. Princeton University Press, Princeton, N.J., 1977. With notes by J. Milnor and M. F. Atiyah, Annals of Mathematics Studies, No. 88.

[KW09] John R. Klein and Bruce Williams. The refined transfer, bundle structures, and algebraic $K$-theory. J. Topol., 2(2):321-345, 2009.

[Lac69] R. C. Lacher. Cell-like mappings. I. Pacific J. Math., 30:717-731, 1969.

[Lüc87] Wolfgang Lück. The transfer maps induced in the algebraic $K_{0}$ - and $K_{1}$ groups by a fibration. II. J. Pure Appl. Algebra, 45(2):143-169, 1987.

[Lüc89] Wolfgang Lück. Transformation groups and algebraic K-theory. SpringerVerlag, Berlin, 1989.

[May99] J. P. May. A concise course in algebraic topology. Chicago Lectures in Mathematics. The university of Chicago press, Chicago, 1999.

[Maz64] Barry Mazur. The method of infinite repetition in pure topology. I. Ann. of Math. (2), 80:201-226, 1964.

[Mil64] J. Milnor. Microbundles. I. Topology, 3(suppl. 1):53-80, 1964.

[MM79] Ib Madsen and R. James Milgram. The classifying spaces for surgery and cobordism of manifolds. Princeton University Press, Princeton, N.J., 1979.

[Qui69] F. Quinn. A geometric formulation of surgery. PhD thesis, Princeton, 1969.

[Seg74] Graeme Segal. Categories and cohomology theories. Topology, 13:293312, 1974.

[Sie70] L. C. Siebenmann. Infinite simple homotopy types. Nederl. Akad. Wetensch. Proc. Ser. A 73 = Indag. Math., 32:479-495, 1970.

[Ste07] W. Steimle. Whitehead-Torsion und Faserungen. arXiv:0706.3973, 2007.

[Str68] Arne Strøm. Note on cofibrations. II. Math. Scand., 22:130-142 (1969), 1968.

[Tu165] Patricia Tulley. On regularity in Hurewicz fiber spaces. Trans. Amer. Math. Soc., 116:126-134, 1965.

[Wal66] C. T. C. Wall. Classification problems in differential topology. IV. Thickenings. Topology, 5:73-94, 1966. 
[Wal78] Friedhelm Waldhausen. Algebraic $K$-theory of topological spaces. I. In Algebraic and geometric topology (Proc. Sympos. Pure Math., Stanford Univ., Stanford, Calif., 1976), Part 1, Proc. Sympos. Pure Math., XXXII, pages 35-60. Amer. Math. Soc., Providence, R.I., 1978.

[Wal82] Friedhelm Waldhausen. Algebraic $K$-theory of spaces, a manifold approach. In Current trends in algebraic topology, Part 1 (London, Ont., 1981), pages 141-184. Amer. Math. Soc., Providence, R.I., 1982.

[Wal85] Friedhelm Waldhausen. Algebraic $K$-theory of spaces. In Algebraic and geometric topology (New Brunswick, N.J., 1983), pages 318-419. SpringerVerlag, Berlin, 1985.

[Wei02] Michael Weiss. Excision and restriction in controlled $K$-theory. Forum Math., 14(1):85-119, 2002.

[Whi78] George W. Whitehead. Elements of homotopy theory. Springer-Verlag, New York, 1978.

[WJR08] F. Waldhausen, B. Jahren, and J. Rognes. Spaces of PL manifolds and categories of simple maps. preprint, 2008.

[WW95] Michael Weiss and Bruce Williams. Assembly. In Novikov conjectures, index theorems and rigidity, Vol. 2 (Oberwolfach, 1993), pages 332-352. Cambridge Univ. Press, Cambridge, 1995.

[WW01] Michael Weiss and Bruce Williams. Automorphisms of manifolds. In Surveys on surgery theory, Vol. 2, volume 149 of Ann. of Math. Stud., pages 165-220. Princeton Univ. Press, Princeton, NJ, 2001. 
